\title{
Semigroups of rectangular matrices under a sandwich operation
}

\author{
Igor Dolinka \\ Department of Mathematics and Informatics \\ University of Novi Sad, Trg Dositeja Obradovića 4, 21101 Novi Sad, Serbia \\ dockie@dmi.uns.ac.rs \\ James East \\ Centre for Research in Mathematics; School of Computing, Engineering and Mathematics \\ Western Sydney University, Locked Bag 1797, Penrith NSW 2751, Australia \\ J.East @ WesternSydney . edu . au
}

August 16, 2016

\begin{abstract}
Let $\mathcal{M}_{m n}=\mathcal{M}_{m n}(\mathbb{F})$ denote the set of all $m \times n$ matrices over a field $\mathbb{F}$, and fix some $n \times m$ matrix $A \in \mathcal{M}_{n m}$. An associative operation $\star$ may be defined on $\mathcal{M}_{m n}$ by $X \star Y=X A Y$ for all $X, Y \in \mathcal{M}_{m n}$, and the resulting sandwich semigroup is denoted $\mathcal{M}_{m n}^{A}=\mathcal{M}_{m n}^{A}(\mathbb{F})$. These semigroups are closely related to Munn rings, which are fundamental tools in the representation theory of finite semigroups. In this article, we study $\mathcal{M}_{m n}^{A}$ as well as its subsemigroups $\operatorname{Reg}\left(\mathcal{M}_{m n}^{A}\right)$ and $\mathcal{E}_{m n}^{A}$ (consisting of all regular elements and products of idempotents, respectively), as well as the ideals of $\operatorname{Reg}\left(\mathcal{M}_{m n}^{A}\right)$. Among other results, we: characterise the regular elements; determine Green's relations and preorders; calculate the minimal number of matrices (or idempotent matrices, if applicable) required to generate each semigroup we consider; and classify the isomorphisms between finite sandwich semigroups $\mathcal{M}_{m n}^{A}\left(\mathbb{F}_{1}\right)$ and $\mathcal{M}_{k l}^{B}\left(\mathbb{F}_{2}\right)$. Along the way, we develop a general theory of sandwich semigroups in a suitably defined class of partial semigroups related to Ehresmann-style "arrows only" categories; we hope this framework will be useful in studies of sandwich semigroups in other categories. We note that all our results have applications to the variants $\mathcal{M}_{n}^{A}$ of the full linear monoid $\mathcal{M}_{n}$ (in the case $m=n$ ), and to certain semigroups of linear transformations of restricted range or kernel (in the case that $\operatorname{rank}(A)$ is equal to one of $m, n$ ).

Keywords: Matrix semigroups, sandwich semigroups, variant semigroups, idempotents, generators, rank, idempotent rank, Munn rings, generalised matrix algebras.
\end{abstract}

MSC: 15A30; 20M20; 20M10; 20M17.

\section{Introduction}

In the classical representation theory of finite semigroups, a key role is played by the so-called Munn rings. These are rings of $m \times n$ matrices (where $m$ and $n$ need not be equal) with the familiar addition operation but with a sandwich multiplication defined by $X \star Y=X A Y$, where $A$ is a fixed $n \times m$ matrix. These rings are so named, because of Douglas Munn's 1955 paper [68], in which it was shown that: (1) the representation theory of a finite semigroup is determined by the representations of certain completely 0 -simple semigroups arising from its ideal structure, and (2) the semigroup algebra of such a finite completely 0-simple semigroup is isomorphic to an appropriate Munn ring over the group algebra of a naturally associated maximal subgroup; conditions were also given for such a Munn ring to be semisimple. (Here, the sandwich matrix A arises from the celebrated Rees structure theorem [81] for completely 0-simple semigroups.) Since their introduction in [68], Munn rings have been studied by numerous authors, and continue to heavily inflence the theory of semigroup representations: for classical studies, see $12,14,37,54,62,64,68,70,76$; for modern accounts, see for example [1, 29, 47, 75, 78, 79, 85, 86, and especially the monographs $73,74,77,82,87$.

In the same year as Munn's article [68] was published, William Brown introduced the so-called generalised matrix algebras [5], motivated by a connection with classical groups [3, 6, 95]. These generalised matrix 
algebras are again rings of $m \times n$ matrices over a field, with multiplication determined by a fixed $n \times m$ sandwich matrix. Whereas the sandwich matrix in a Munn ring is taken to be the structure matrix of a completely 0-simple semigroup (and so has a certain prescribed form), Brown considered arbitrary sandwich matrices. As with Munn rings, these generalised matrix algebras have influenced representation theory to this day, and have been studied by numerous authors; see for example $21,30,35,51,52,55,92,96,97]$.

Shortly after the Munn and Brown articles [5, 68] appeared, Evgeny Lyapin's early monograph on semigroups [57] was published. In [57, Chapter VII], we find a number of interesting semigroup constructions, including the following. Let $V$ and $W$ be arbitrary non-empty sets, and let $\theta: W \rightarrow V$ be an arbitrary (but fixed) function. Then the set $\mathcal{T}(V, W)$ of all functions $V \rightarrow W$ forms a semigroup, denoted $\mathcal{T}^{\theta}(V, W)$, under the operation $\star_{\theta}$ defined by $f \star_{\theta} g=f \circ \theta \circ g$. If it is assumed that $V$ and $W$ are vector spaces (over the same field) and $\theta$ a linear transformation, then the subset $\mathcal{L}(V, W) \subseteq \mathcal{T}(V, W)$ of all linear transformations $V \rightarrow W$ is a subsemigroup of $\mathcal{T}^{\theta}(V, W)$. This subsemigroup, denoted $\mathcal{L}^{\theta}(V, W)$ and referred to as a linear sandwich semigroup, is clearly isomorphic to the underlying multiplicative semigroup of an associated generalised matrix algebra [5]. As noted above, the addition on a generalised matrix algebra is just the usual operation, so these linear sandwich semigroups capture and isolate (in a sense) the more complex of the operations on the algebras.

The sandwich semigroups $\mathcal{T}^{\theta}(V, W)$ were first investigated in a series of articles by Magill and Subbiah [58 60], and more recent studies may be found in [7, 65, 90, 93]; most of these address structural concerns such as (von Neumann) regularity, Green's relations, ideals, classification up to isomorphism, and so on. The linear sandwich semigroups $\mathcal{L}^{\theta}(V, W)$ have received less attention, though they have also been studied by a number of authors $9,48,49,66$, with studies again focusing on basic structural properties. This is regrettable, because these semigroups display a great deal of algebraic and combinatorial charm, as we hope to show in the current article. It is therefore our purpose to carry out a systematic investigation of the linear sandwich semigroups, bringing their study up to date, and focusing on modern themes, especially combinatorial invariant theory. As does Brown [5], we focus on the case that $V$ and $W$ are finite dimensional; in fact, we study the equivalent sandwich semigroups $\mathcal{M}_{m n}^{A}=\mathcal{M}_{m n}^{A}(\mathbb{F})$ consisting of all $m \times n$ matrices over the field $\mathbb{F}$ under the operation $\star_{A}$ defined by $X \star_{A} Y=X A Y$, where $A$ is a fixed $n \times m$ matrix.

We speculate that the difficulty (until now) of systematically investigating the linear sandwich semigroups may be due to the lack of a consistent theoretical framework for studying sandwich semigroups in more generality. In the case that $V=W$, the sets $\mathcal{T}(V, W)$ and $\mathcal{L}(V, W)$ are themselves semigroups (under composition); these are the full transformation semigroup $\mathcal{T}_{V}[23,28,32,34,42,43,45,65]$ and the general linear monoid $\mathcal{L}_{V}, 2,15,16,18,20,25,33,53,74,80,94$, , respectively. In turn, the semigroups $\mathcal{T}^{\theta}(V, V)$ and $\mathcal{L}^{\theta}(V, V)$ are special cases of the semigroup variant construction. The variant of a semigroup $S$ with respect to an element $a \in S$ is the semigroup $S^{a}=\left(S, \star_{a}\right)$, with operation defined by $x \star_{a} y=x a y$. Variants were first explicitly studied by Hickey in the 1980s [38, 39, though (as noted above) the idea goes back to Lyapin's monograph [57]; a more recent study may be found in [50]. The current authors developed the general theory of variants further in [19], and then used this as a starting point to explore the variants of the finite full transformation semigroups, obtaining a great deal of algebraic and combinatorial information about these semigroups. Unfortunately, the theory of semigroup variants does not help with studying the more general sandwich semigroups $\mathcal{T}^{\theta}(V, W)$ and $\mathcal{L}^{\theta}(V, W)$, since the underlying sets $\mathcal{T}(V, W)$ and $\mathcal{L}(V, W)$ are not even semigroups if $V \neq W$. One of the main goals of the current article, therefore, is to develop an appropriate general framework for working with arbitrary sandwich semigroups. Namely, if $V$ and $W$ are objects in a (locally) small category $\mathscr{C}$, and if $\theta \in \operatorname{Hom}(W, V)$ is some fixed morphism, then the set $\operatorname{Hom}(V, W)$ becomes a semigroup under the sandwich operation defined by $f \star_{\theta} g=f \circ \theta \circ g$, for $f, g \in \operatorname{Hom}(V, W)$. (In the case that $V=W$ and $\theta$ is the identity morphism, this construction reduces to the usual endomorphism monoid $\operatorname{End}(V)$.) The semigroups $\mathcal{T}^{\theta}(V, W)$ and $\mathcal{L}^{\theta}(V, W)$ arise when $\mathscr{C}$ is the category of sets (and mappings) or vector spaces (and linear transformations), respectively. In order to develop a general theory of sandwich semigroups in such categories, we first explain how many important semigroup theoretical techniques extend to the more general categorical setting; we note that there is only a little overlap with the theory of Green's relations in categories developed in [56], which focuses on issues more relevant to representation theory. In order to avoid any confusion arising from terminology conflicts between semigroup and category theory, rather than speak of (locally small) categories, we focus on the equivalently defined class of partial semigroups, which are related to Ehresmann-style "arrows only" categories [24]. We hope that the general theory we develop will prove to be a useful starting point for future studies of sandwich 
semigroups in other categories.

The article is organised as follows. In Section 2, we develop a general theory of sandwich semigroups in partial semigroups (i.e., locally finite categories), extending certain important semigroup theoretic notions (such as Green's relations, regularity and stability, the definitions of which are given in Section 2 ) to the more general context. In Section 3, we gather results on the partial semigroup $\mathcal{M}=\mathcal{M}(\mathbb{F})$ of all (finite dimensional) matrices over the field $\mathbb{F}$, mainly focusing on regularity, stability and Green's relations, and we state some well-known results on (idempotent) generation and ideals of the general linear monoids $\mathcal{M}_{n}$. We begin our investigation of the linear sandwich semigroups $\mathcal{M}_{m n}^{A}$ in Section 4 , the main results of this section being: a characterisation of the regular elements (Proposition 4.3); a description of Green's relations (Theorem 4.5) and the ordering on $\mathscr{D}$-classes (Propositions 4.6, 4.7 and 4.10); a classification of the isomorphism classes of sandwich semigroups over $\mathcal{M}_{m n}$ (Corollary 4.8); and the calculation of $\operatorname{rank}\left(\mathcal{M}_{m n}^{A}\right)$ (Theorems 4.12 and 4.14). (Recall that the rank of a semigroup $S$, denoted $\operatorname{rank}(S)$, is the minimum size of a generating set for $S$.) Section 5 explores the relationship between a sandwich semigroup $\mathcal{M}_{m n}^{A}$ and various (nonsandwich) matrix semigroups, the main structural results being Theorem 5.7 and Propositions 5.8 and 5.11 . We then focus on the regular subsemigroup $P=\operatorname{Reg}\left(\mathcal{M}_{m n}^{A}\right)$ in Section 6 , where we: calculate the size of $P$ and various Green's classes (Proposition 6.2 and Theorem 6.4); classify the isomorphism classes of finite linear sandwich semigroups (Theorem 6.5); and calculate $\operatorname{rank}(P)$ (Theorem 6.10). In Section 7, we investigate the idempotent generated subsemigroup $\mathcal{E}_{m n}^{A}$ of $\mathcal{M}_{m n}^{A}$, where we: enumerate the idempotents of $\mathcal{M}_{m n}^{A}$ (Proposition 7.2); show that $\mathcal{E}_{m n}^{A}$ consists of $P \backslash D$ and the idempotents from $D$, where $D$ is the maximal $\mathscr{D}$-class (Theorem 7.3 ; and calculate $\operatorname{rank}\left(\mathcal{E}_{m n}^{A}\right)$ and $\operatorname{idrank}\left(\mathcal{E}_{m n}^{A}\right)$, showing in particular that these are equal (Theorem 7.5). (The idempotent rank of an idempotent generated semigroup $S$, denoted $\operatorname{idrank}(S)$, is defined similarly to the rank, but with respect to idempotent generating sets for $S$.) Finally, in Section 8, we classify the proper ideals of $P$, showing that these are idempotent generated, and calculating their ranks and idempotent ranks, which are again equal (Theorem 8.1). We note that all our results have applications to the variants $\mathcal{M}_{n}^{A}$ of the full linear monoid $\mathcal{M}_{n}$ (in the case $m=n$ ), and to certain semigroups of linear transformations of restricted range or kernel (in the case that $\operatorname{rank}(A)$ is equal to one of $m, n$; see Remarks 4.2 and 5.3 .

\section{Sandwich semigroups from partial semigroups}

Recall that our main interest is in the linear sandwich semigroups $\mathcal{M}_{m n}^{A}=\mathcal{M}_{m n}^{A}(\mathbb{F})$. The underlying set of $\mathcal{M}_{m n}^{A}$ is $\mathcal{M}_{m n}$, the set of all $m \times n$ matrices over the field $\mathbb{F}$, which is not itself a semigroup (unless $m=n$ ). However, $\mathcal{M}_{m n}$ is contained in $\mathcal{M}$, the set of all (finite dimensional) matrices over $\mathbb{F}$. While $\mathcal{M}$ is still not a semigroup, it does have the structure of a (small) category. As we will see, in order to understand the linear sandwich semigroups $\mathcal{M}_{m n}^{A}$, we need to move beyond just $m \times n$ (and $n \times m$ ) matrices, and gain a fuller understanding of the whole category $\mathcal{M}$. Some (but not all) of what we need to know about $\mathcal{M}$ is true in a larger class of categories, and more general structures we call partial semigroups, so we devote this section to the development of the general theory of these structures. We begin with the definitions.

Definition 2.1. A partial semigroup is a 5 -tuple $(S, \cdot, I, \lambda, \rho)$ consisting of a set $S$, a partial binary operation $(x, y) \mapsto x \cdot y$ (defined on some subset of $S \times S)$, a set $I$, and functions $\lambda, \rho: S \rightarrow I$, such that, for all $x, y, z \in S$,

(i) $x \cdot y$ is defined if and only if $\rho(x)=\lambda(y)$,

(ii) if $x \cdot y$ is defined, then $\lambda(x \cdot y)=\lambda(x)$ and $\rho(x \cdot y)=\rho(y)$,

(iii) if $x \cdot y$ and $y \cdot z$ are defined, then $(x \cdot y) \cdot z=x \cdot(y \cdot z)$.

We say that a partial semigroup $(S, \cdot, I, \lambda, \rho)$ is monoidal if in addition to (i-iii),

(iv) there exists a function $I \rightarrow S: i \mapsto e_{i}$ such that, for all $x \in S, x \cdot e_{\rho(x)}=x=e_{\lambda(x)} \cdot x$.

We say that a partial semigroup $(S, \cdot, I, \lambda, \rho)$ is regular if in addition to (i-iii),

(v) for all $x \in S$, there exists $y \in S$ such that $x=x \cdot y \cdot x$ and $y=y \cdot x \cdot y$. 
Remark 2.2. We note that conditions (i-iv) amount to one of several equivalent ways to define (small) categories in an "arrows only" fashion. See for example Ehresmann's monograph [24], and also [41] for a historical discussion of the connections between category theory and (inverse) semigroup theory.

For a partial semigroup $(S, \cdot, I, \lambda, \rho)$, and for $i, j \in I$, we write

$$
S_{i j}=\{x \in S: \lambda(x)=i, \rho(x)=j\} \quad \text { and } \quad S_{i}=S_{i i} .
$$

So $S=\bigcup_{i, j \in I} S_{i j}$. Note that if $x \in S$, then $x \cdot x$ is defined if and only if $\lambda(x)=\rho(x)$. It follows that $S_{i}$ is a semigroup with respect to the induced binary operation (the restriction of - to $S_{i} \times S_{i}$ ) for each $i \in I$, but that $S_{i j}$ is not if $i \neq j$. We will often slightly abuse notation and refer to "the partial semigroup $S$ " if the rest of the data $(S, \cdot, I, \lambda, \rho)$ is clear from context. We also note that in what follows, we could allow $S$ and $I$ to be classes (rather than insist on them being sets); but we would still require $S_{i j}$ to be a set for each $i, j \in I$.

Note that, as is the case with semigroups, condition (v) is equivalent to the (ostensibly) weaker condition:

$(\mathrm{v})^{\prime}$ for all $x \in S$, there exists $z \in S$ such that $x=x \cdot z \cdot x$.

Indeed, with $z$ as in $(\mathrm{v})^{\prime}$, one easily checks that $y=z \cdot x \cdot z$ satisfies the condition of $(\mathrm{v})$.

If $S$ is monoidal, then $S_{i}$ is a monoid with identity $e_{i} \in S_{i}$ for each $i$. If $S$ is not monoidal, then $S$ may be embedded in a monoidal partial semigroup $S^{(1)}$ as follows: for each $i \in I$ we adjoin an element $e_{i}$ to $S_{i}$ and declare that $x \cdot e_{i}=x$ and $e_{i} \cdot y=y$ for all $x, y \in S$ with $\rho(x)=i$ and $\lambda(y)=i$, if such an element $e_{i} \in S_{i}$ does not already exist. In particular, if $S$ is monoidal, then $S=S^{(1)}$.

Obviously any semigroup is a partial semigroup (with $|I|=1$ ); in particular, all results we prove in this section concerning partial semigroups hold for semigroups. A great number of non-semigroup examples exist, but we will limit ourselves to describing just a few.

Example 2.3. As a trivial example, let $\left\{S_{i}: i \in I\right\}$ be any set of pairwise disjoint semigroups. Then $S=\bigcup_{i \in I} S_{i}$ is a partial semigroup where we define $\lambda, \rho: S \rightarrow I$ by $\lambda(x)=\rho(x)=i$ for each $i \in I$ and $x \in S_{i}$, and $x \cdot y$ is defined if and only if $x, y \in S_{i}$ for some $i$, in which case $x \cdot y$ is just the product of $x, y$ in $S_{i}$. Note that this $S$ is regular (resp., monoidal) if and only if each $S_{i}$ is regular (resp., a monoid).

Example 2.4. Let $\mathscr{X}$ be some set, and $\mathcal{P}(\mathscr{X})=\{A: A \subseteq \mathscr{X}\}$ the power set of $\mathscr{X}$. The set $T_{\mathscr{X}}=$ $\{(B, f, A): A, B \subseteq \mathscr{X}, f$ is a function $A \rightarrow B\}$ is a regular monoidal partial semigroup. We define $I=$ $\mathcal{P}(\mathscr{X})$, and $\lambda(B, f, A)=B$ and $\rho(B, f, A)=A$, with $(D, g, C) \cdot(B, f, A)$ defined if and only if $B=C$, in which case $(D, g, C) \cdot(B, f, A)=(D, g \circ f, A)$.

The previous example may be extended in a number of ways, by replacing functions $f: A \rightarrow B$ by other objects such as binary relations [8, 91], partial functions [11, 88], partial bijections [10], block bijections [26], partial braids 22], partitions [61], Brauer diagrams [3], etc., or by assuming the functions $f: A \rightarrow B$ preserve some kind of algebraic or geometric structure on the sets $A, B$. The main example we will concentrate on in this article is as follows.

Example 2.5. Let $\mathbb{F}$ be a field, and write $\mathcal{M}=\mathcal{M}(\mathbb{F})$ for the set of all (finite dimensional, non-empty) matrices over $\mathbb{F}$. Then $\mathcal{M}$ has the structure of a regular monoidal partial semigroup. We take $I=\mathbb{N}=$ $\{1,2,3, \ldots\}$ to be the set of all natural numbers and, for $X \in \mathcal{M}$, we define $\lambda(X)$ (resp., $\rho(X)$ ) to be the number of rows (resp., columns) of $X$. For $m, n \in \mathbb{N}, \mathcal{M}_{m n}=\mathcal{M}_{m n}(\mathbb{F})$ denotes the set of all $m \times n$ matrices over $\mathbb{F}$, and forms a semigroup if and only if $m=n$. (Of course, $\mathcal{M}$ is isomorphic to a certain partial semigroup of linear transformations; we will have more to say about this later.)

For the remainder of this section, we fix a partial semigroup $(S, \cdot, I, \lambda, \rho)$, and we write $x y$ for the product $x \cdot y$ (whenever it is defined). Note that we may define a second partial binary operation $\bullet$ on $S$ by

$$
x \bullet y=y \cdot x \quad \text { for each } x, y \in S \text { with } \rho(y)=\lambda(x) .
$$


We see then that $(S, \bullet, I, \rho, \lambda)$ is a partial semigroup (note the swapping of $\lambda$ and $\rho$ ), and we call this the dual partial semigroup to $(S, \cdot, I, \lambda, \rho)$. As is frequently the case in semigroup theory, this duality will allow us to shorten several proofs.

Green's relations and preorders are crucial tools in semigroup theory (for general background on semigroups, see 40,44$]$ ), and we will need to extend these to the partial semigroup setting. If $x, y \in S$, then we say

- $x \leq_{\mathscr{R}} y$ if $x=y a$ for some $a \in S^{(1)}$,

- $x \leq_{\mathscr{L}} y$ if $x=a y$ for some $a \in S^{(1)}$,

- $x \leq \mathscr{J} y$ if $x=a y b$ for some $a, b \in S^{(1)}$.

Note that if $x \leq_{\mathscr{R}} y$ (resp., $x \leq \mathscr{L} y$ ), then $\lambda(x)=\lambda(y)$ (resp., $\rho(x)=\rho(y)$ ). Note also that if $x \leq_{\mathscr{R}} y$, then $u x \leq_{\mathscr{R}} u y$ for any $u \in S$ with $\rho(u)=\lambda(x)$; a dual statement holds for the $\leq \mathscr{L}$ relation. Finally, note that the use of $S^{(1)}$ is merely for convenience since, for example, $x \leq_{\mathscr{R}} y$ means that $x=y$ or $x=y a$ for some $a \in S$. All three of the above relations are preorders (i.e., they are reflexive and transitive). If $\mathscr{K}$ is one of $\mathscr{R}, \mathscr{L}, \mathscr{J}$, we write $\mathscr{K}=\leq \mathscr{K} \cap \geq \mathscr{K}$ for the equivalence relation on $S$ induced by $\mathscr{K}$. So, for example, $x \mathscr{R} y$ if and only if $x=y a$ and $y=x b$ for some $a, b \in S^{(1)}$. We also define equivalence relations

$$
\mathscr{H}=\mathscr{R} \cap \mathscr{L} \quad \text { and } \quad \mathscr{D}=\mathscr{R} \vee \mathscr{L} .
$$

(The join $\varepsilon \vee \eta$ of two equivalences $\varepsilon$ and $\eta$ is the transitive closure of $\varepsilon \cup \eta$, and is itself an equivalence.) It is easy to see that $\mathscr{D} \subseteq \mathscr{J}$. The duality mentioned above means that $x \leq \mathscr{R} y$ in $(S, \cdot, I, \lambda, \rho)$ if and only if $x \leq \mathscr{L} y$ in $(S, \bullet, I, \rho, \lambda)$, and so on.

Analogously to the definition for semigroups [83, Definition A.2.1], we say that the partial semigroup $S$ is stable if for all $x, y \in S$,

$$
x \mathscr{J} x y \Leftrightarrow x \mathscr{R} x y \quad \text { and } \quad x \mathscr{J} y x \Leftrightarrow x \mathscr{L} y x .
$$

The following simple but crucial observation is proved in analogous fashion to the corresponding results for semigroups; see for example [44, Proposition 2.1.3] and [83, Corollary A.2.5].

Lemma 2.6. We have $\mathscr{D}=\mathscr{R} \circ \mathscr{L}=\mathscr{L} \circ \mathscr{R}$. If $S$ is stable, then $\mathscr{D}=\mathscr{J}$.

If $x \in S_{i j}$ and $\mathscr{K}$ is one of $\mathscr{R}, \mathscr{L}, \mathscr{J}, \mathscr{D}, \mathscr{H}$, we write

$$
[x]_{\mathscr{K}}=\{y \in S: x \mathscr{K} y\} \quad \text { and } \quad K_{x}=[x]_{\mathscr{K}} \cap S_{i j}=\left\{y \in S_{i j}: x \mathscr{K} y\right\} .
$$

We call $[x]_{\mathscr{K}}$ (resp., $K_{x}$ ) the $\mathscr{K}$-class of $x$ in $S$ (resp., in $S_{i j}$ ). The next result is reminiscent of Green's Lemma, and may be proved in virtually identical fashion to [44, Lemma 2.2.1].

Lemma 2.7. Let $x, y \in S$.

(i) Suppose $x \mathscr{R} y$, and that $x=y a$ and $y=x b$ where $a, b \in S^{(1)}$. Then the maps $[x]_{\mathscr{L}} \rightarrow[y]_{\mathscr{L}}: w \mapsto w b$ and $[y]_{\mathscr{L}} \rightarrow[x]_{\mathscr{L}}: w \mapsto w a$ are mutually inverse bijections. These maps restrict to mutually inverse bijections $[x]_{\mathscr{H}} \rightarrow[y]_{\mathscr{H}}$ and $[y]_{\mathscr{H}} \rightarrow[x]_{\mathscr{H}}$.

(ii) Suppose $x \mathscr{L} y$, and that $x=$ ay and $y=b x$ where $a, b \in S^{(1)}$. Then the maps $[x]_{\mathscr{R}} \rightarrow[y]_{\mathscr{R}}: w \mapsto b w$ and $[y]_{\mathscr{R}} \rightarrow[x]_{\mathscr{R}}: w \mapsto$ aw are mutually inverse bijections. These maps restrict to mutually inverse bijections $[x]_{\mathscr{H}} \rightarrow[y]_{\mathscr{H}}$ and $[y]_{\mathscr{H}} \rightarrow[x]_{\mathscr{H}}$.

(iii) If $x \mathscr{D} y$, then $\left|[x]_{\mathscr{R}}\right|=\left|[y]_{\mathscr{R}}\right|,\left|[x]_{\mathscr{L}}\right|=\left|[y]_{\mathscr{L}}\right|$ and $\left|[x]_{\mathscr{H}}\right|=\left|[y]_{\mathscr{H}}\right|$.

Note that if $x, y \in S$ are such that $x \mathscr{H} y$, then $\lambda(x)=\lambda(y)$ and $\rho(x)=\rho(y)$. It follows that $[x]_{\mathscr{H}}=H_{x}$ for all $x \in S$.

Lemma 2.8. Let $x, y \in S_{i j}$.

(i) Suppose $x \mathscr{R} y$, and that $x=y a$ and $y=x b$ where $a, b \in S^{(1)}$. Then the maps $L_{x} \rightarrow L_{y}: w \mapsto w b$ and $L_{y} \rightarrow L_{x}: w \mapsto w a$ are mutually inverse bijections. These maps restrict to mutually inverse bijections $H_{x} \rightarrow H_{y}$ and $H_{y} \rightarrow H_{x}$. 
(ii) Suppose $x \mathscr{L} y$, and that $x=$ ay and $y=b x$ where $a, b \in S^{(1)}$. Then the maps $R_{x} \rightarrow R_{y}: w \mapsto$ bw and $R_{y} \rightarrow R_{x}: w \mapsto$ aw are mutually inverse bijections. These maps restrict to mutually inverse bijections $H_{x} \rightarrow H_{y}$ and $H_{y} \rightarrow H_{x}$.

(iii) If $x \mathscr{D} y$, then $\left|R_{x}\right|=\left|R_{y}\right|,\left|L_{x}\right|=\left|L_{y}\right|$ and $\left|H_{x}\right|=\left|H_{y}\right|$.

Proof. Suppose $x \mathscr{R} y$, and that $x=y a$ and $y=x b$ where $a, b \in S^{(1)}$. We first show that the map $f: L_{x} \rightarrow S: w \mapsto w b$ does indeed map $L_{x}$ into $L_{y}$. With this in mind, let $w \in L_{x}$. We already know that $w b \in[y]_{\mathscr{L}}$, by Lemma 2.7(i). Also, $w=u x$ for some $u \in S^{(1)}$, since $w \mathscr{L} x$. Now, $\lambda(w b)=\lambda(w)=i$, and also $\rho(w b)=\rho(u x b)=\rho(u y)=\rho(y)=j$, showing that $w b \in[y]_{\mathscr{L}} \cap S_{i j}=L_{y}$, as required. By symmetry, it follows that $g: L_{y} \rightarrow S: w \mapsto w a$ maps $L_{y}$ into $L_{x}$. By Lemma 2.7(i), we see that $f \circ g$ and $g \circ f$ are the identity maps on their respective domains. This completes the proof of (i).

Next, note that (ii) follows from (i) by duality. Now suppose $x \mathscr{D} y$. So $x \mathscr{R} z \mathscr{L} y$ for some $z \in S$. Since $x \mathscr{R} z$, it follows that $\lambda(z)=\lambda(x)=i$; similarly, $\rho(z)=j$, so in fact, $z \in S_{i j}$. In particular, $R_{x}=R_{z}$ and $L_{y}=L_{z}$. The statement about cardinalities then follows from parts (i) and (ii).

As is the case for semigroups 40,44], Lemma 2.6 means that the elements of a $\mathscr{D}$-class $D$ of $S$ or $S_{i j}$ may be grouped together in a rectangular array of cells, which (for continuity with semigroup theory) we call an eggbox. We place all elements from $D$ in a box in such a way that $\mathscr{R}$-related (resp., $\mathscr{L}$-related) elements are in the same row (resp., column), and $\mathscr{H}$-related elements in the same cell. An example is given in Figure 2 below for a $\mathscr{D}$-class of the linear partial semigroup $\mathcal{M}\left(\mathbb{Z}_{3}\right)$.

We now come to the definition of the main objects of our study, the sandwich semigroups.

Definition 2.9. Let $(S, \cdot, I, \lambda, \rho)$ be a partial semigroup. Fix some $a \in S_{j i}$, where $i, j \in I$. Define a binary operation $\star_{a}$ on $S_{i j}$ by $x \star_{a} y=x a y$ for each $x, y \in S_{i j}$. It is easily checked that $\star_{a}$ is associative. We denote by $S_{i j}^{a}=\left(S_{i j}, \star_{a}\right)$ the semigroup obtained in this way, and call $S_{i j}^{a}$ the sandwich semigroup of $S_{i j}$ with respect to $a$. (Note that when $i=j, S_{i j}^{a}=S_{i}^{a}$ is the well-known variant 38, 39,50 of $S_{i}$ with respect to $a \in S_{i}$.)

Recall that an element $x$ of a semigroup $T$ is regular if $x=x y x$ and $y=y x y$ for some $y \in T$ (or, equivalently, if $x=x z x$ for some $z \in T$ ). The set of all regular elements of $T$ is denoted by $\operatorname{Reg}(T)$, and we say $T$ is regular if $T=\operatorname{Reg}(T)$. (In general, $\operatorname{Reg}(T)$ need not even be a subsemigroup of $T$.) Of crucial importance is that if any element of a $\mathscr{D}$-class $D$ of a semigroup $T$ is regular, then every element of $D$ is regular, in which case every element of $D$ is $\mathscr{L}$-related to at least one idempotent (and also $\mathscr{R}$-related to a possibly different idempotent); the $\mathscr{H}$-class $H_{e}$ of an idempotent $e \in E(T)=\left\{x \in T: x=x^{2}\right\}$ is a group, and $H_{e} \cong H_{f}$ for any two $\mathscr{D}$-related idempotents $e, f \in E(T)$. When drawing eggbox diagrams, group $\mathscr{H}$-classes are usually shaded grey (see for example Figure 3]. See [40, 44] for more details.

If $S$ is a regular partial semigroup, then the sandwich semigroups $S_{i j}^{a}$ need not be regular themselves (although all of the semigroups $S_{i}$ are), but the set $\operatorname{Reg}\left(S_{i j}^{a}\right)$ of all regular elements of $S_{i j}^{a}$ forms a subsemigroup, as we now show.

Proposition 2.10. Let $(S, \cdot, I, \lambda, \rho)$ be a regular partial semigroup. Then $\operatorname{Reg}\left(S_{i j}^{a}\right)$ is a subsemigroup of $S_{i j}^{a}$ for all $i, j \in I$ and $a \in S_{j i}$.

Proof. Let $x, y \in \operatorname{Reg}\left(S_{i j}^{a}\right)$, so $x=x a u a x$ and $y=$ yavay for some $u, v \in S_{i j}$. Since $S$ is regular, there exists $w \in S$ such that (auaxayava) $w($ auaxayava $)=($ auaxayava $)$. Then

$$
\begin{aligned}
(x a y) a(\text { vawau }) a(x a y) & =(\text { xauaxay }) a(\text { vawau }) \text { (xayavay }) \\
& =x(\text { auaxayava }) w(\text { auaxayava }) y=x(\text { auaxayava }) y=x a y,
\end{aligned}
$$

showing that $\left(x \star_{a} y\right) \star\left(v \star_{a} w \star_{a} u\right) \star_{a}\left(x \star_{a} y\right)=x \star_{a} y$, and $x \star_{a} y \in \operatorname{Reg}\left(S_{i j}^{a}\right)$.

In order to say more about the regular elements and Green's relations of the sandwich semigroup $S_{i j}^{a}$, we define the sets

$$
P_{1}^{a}=\left\{x \in S_{i j}: x a \mathscr{R} x\right\}, \quad P_{2}^{a}=\left\{x \in S_{i j}: a x \mathscr{L} x\right\}, \quad P_{3}^{a}=\left\{x \in S_{i j}: a x a \mathscr{J} x\right\}, \quad P^{a}=P_{1}^{a} \cap P_{2}^{a} .
$$


The next result explains the relationships that hold between these sets; the various inclusions are pictured in Figure 1.
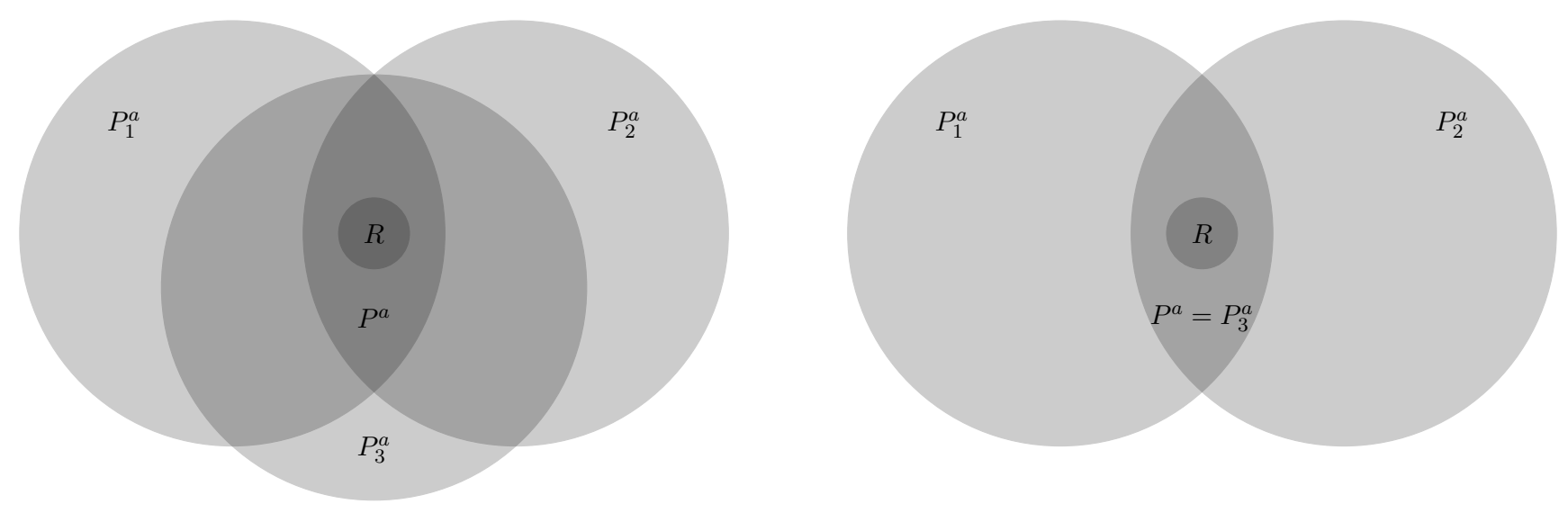

Figure 1: Venn diagrams illustrating the various relationships between the sets $P_{1}^{a}, P_{2}^{a}, P_{3}^{a}, P^{a}=P_{1}^{a} \cap P_{2}^{a}$ and $\operatorname{Reg}\left(S_{i j}^{a}\right)$ in the general case (left) and the stable case (right); for clarity, we have written $R=\operatorname{Reg}\left(S_{i j}^{a}\right)$.

Proposition 2.11. Let $(S, \cdot, I, \lambda, \rho)$ be a partial semigroup, and fix $i, j \in I$ and $a \in S_{j i}$. Then

(i) $\operatorname{Reg}\left(S_{i j}^{a}\right) \subseteq P^{a} \subseteq P_{3}^{a}$,

(ii) $P^{a}=P_{3}^{a}$ if $S$ is stable.

Proof. If $x \in \operatorname{Reg}\left(S_{i j}^{a}\right)$, then $x=x a y a x$ for some $y \in S_{i j}$, giving $x \mathscr{R} x a$ and $x \mathscr{L} a x$, so that $x \in P_{1}^{a} \cap P_{2}^{a}=P^{a}$. Next, suppose $x \in P^{a}=P_{1}^{a} \cap P_{2}^{a}$, so $x=x a v=u a x$ for some $u, v \in S^{(1)}$. It follows that $x=$ uaxav, so $x \mathscr{J}$ axa and $x \in P_{3}^{a}$. This completes the proof of (i).

Now suppose $S$ is stable, and let $x \in P_{3}^{a}$. So $x=$ uaxav for some $u, v \in S^{(1)}$. It then follows that $x \mathscr{J} x a$ and $x \mathscr{J} a x$. By stability, it follows that $x \mathscr{R} x a$ and $x \mathscr{L} a x$, so that $x \in P_{1}^{a} \cap P_{2}^{a}=P^{a}$, completing the proof of (ii).

Remark 2.12. The assumption of regularity (resp., stability) could be greatly weakened in Proposition 2.10 (resp., Proposition 2.11(ii)). However, because the linear partial semigroup $\mathcal{M}$ is regular and stable (see Lemmas 3.1 and 3.2 , we will not pursue this thought any further.

We now show how the sets $P_{1}^{a}, P_{2}^{a}, P_{3}^{a}$ and $P^{a}=P_{1}^{a} \cap P_{2}^{a}$ may be used to relate Green's relations on the sandwich semigroups $S_{i j}^{a}$ to the corresponding relations on $S$. To avoid confusion, if $\mathscr{K}$ is one of $\mathscr{R}, \mathscr{L}, \mathscr{J}$, $\mathscr{D}, \mathscr{H}$, we write $\mathscr{K}^{a}$ for the Green's $\mathscr{K}$-relation on $S_{i j}^{a}$. So, for example, if $x, y \in S_{i j}$, then

- $x \mathscr{R}^{a} y$ if and only if $[x=y]$ or $\left[x=y \star_{a} u=y a u\right.$ and $y=x \star_{a} v=x a v$ for some $\left.u, v \in S_{i j}\right]$.

It is then clear that $\mathscr{R}^{a} \subseteq \mathscr{R}$, and the analogous statement is true for all of the other Green's relations. If $x \in S_{i j}$, we write $K_{x}^{a}=\left\{y \in S_{i j}: x \mathscr{K}^{a} y\right\}$ for the $\mathscr{K}^{a}$-class of $x$ in $S_{i j}^{a}$. Since $\mathscr{K}^{a} \subseteq \mathscr{K}$, it follows that $K_{x}^{a} \subseteq K_{x}$ for all $x \in S_{i j}$.

Theorem 2.13. Let $(S, \cdot, I, \lambda, \rho)$ be a partial semigroup, and let $a \in S_{j i}$ where $i, j \in I$. If $x \in S_{i j}$, then

(i) $R_{x}^{a}= \begin{cases}R_{x} \cap P_{1}^{a} & \text { if } x \in P_{1}^{a} \\ \{x\} & \text { if } x \in S_{i j} \backslash P_{1}^{a},\end{cases}$

(ii) $L_{x}^{a}= \begin{cases}L_{x} \cap P_{2}^{a} & \text { if } x \in P_{2}^{a} \\ \{x\} & \text { if } x \in S_{i j} \backslash P_{2}^{a},\end{cases}$

(iii) $H_{x}^{a}= \begin{cases}H_{x} & \text { if } x \in P^{a} \\ \{x\} & \text { if } x \in S_{i j} \backslash P^{a},\end{cases}$ (iv) $D_{x}^{a}= \begin{cases}D_{x} \cap P^{a} & \text { if } x \in P^{a} \\ L_{x}^{a} & \text { if } x \in P_{2}^{a} \backslash P_{1}^{a} \\ R_{x}^{a} & \text { if } x \in P_{1}^{a} \backslash P_{2}^{a} \\ \{x\} & \text { if } x \in S_{i j} \backslash\left(P_{1}^{a} \cup P_{2}^{a}\right),\end{cases}$

(v) $J_{x}^{a}= \begin{cases}J_{x} \cap P_{3}^{a} & \text { if } x \in P_{3}^{a} \\ D_{x}^{a} & \text { if } x \in S_{i j} \backslash P_{3}^{a} .\end{cases}$ 
Further, if $x \in S_{i j} \backslash P^{a}$, then $H_{x}^{a}=\{x\}$ is a non-group $\mathscr{H}^{a}$-class of $S_{i j}^{a}$.

Proof. The proof of [19, Proposition 3.2] may easily be adapted to prove (i-iv) and the final statement about $\mathscr{H}^{a}$-classes. We now prove (v). Let $x \in S_{i j}$.

Suppose $y \in J_{x}^{a} \backslash\{x\}$. So one of (a-c) and one of (d-f) holds:

(a) $y=\operatorname{sax}$ for some $s \in S_{i j}$,

(b) $y=x a t$ for some $t \in S_{i j}$,

(c) $y=$ saxat for some $s, t \in S_{i j}$, (d) $x=$ uay for some $u \in S_{i j}$,

(e) $x=y a v$ for some $v \in S_{i j}$,

(f) $x=$ uayav for some $u, v \in S_{i j}$.

Suppose first that (a) and (d) hold. Then $x \mathscr{L}^{a} y$. Since $x \neq y$, we deduce that $x \in P_{2}^{a}$ by (ii). Since $L_{x}^{a}=L_{y}^{a}$, we also have $y \in P_{2}^{a}$. Similarly, if (b) and (e) hold, then $x \mathscr{R}^{a} y$ and $x, y \in P_{1}^{a}$. One may check that any other combination of $(\mathrm{a}-\mathrm{c})$ and $(\mathrm{d}-\mathrm{f})$ implies $x, y \in P_{3}^{a}$. For example, if (a) and (e) hold, then

$$
y=\operatorname{sax}=s(a y a) v \quad \text { and } \quad x=y a v=s(a x a) v .
$$

In particular, we have shown that $\left|J_{x}^{a}\right| \geq 2$ implies $x \in P_{1}^{a} \cup P_{2}^{a} \cup P_{3}^{a}$. By the contrapositive of this last statement, if $z \in S_{i j} \backslash\left(P_{1}^{a} \cup P_{2}^{a} \cup P_{3}^{a}\right)$, then $J_{z}^{a}=\{z\}=D_{z}^{a}$, with the last equality following from (iv).

Next, suppose $x \in P_{1}^{a} \backslash P_{3}^{a}$. In particular, $x \notin P_{2}^{a}$ since $P_{1}^{a} \cap P_{2}^{a} \subseteq P_{3}^{a}$ by Proposition 2.11(i). Since $\mathscr{D}^{a} \subseteq \mathscr{J}^{a}$, we have $D_{x}^{a} \subseteq J_{x}^{a}$. Conversely, suppose $y \in J_{x}^{a}$. We must show that $y \in D_{x}^{a}$. If $y=x$, then we are done, so suppose $y \neq x$. As above, one of $(\mathrm{a}-\mathrm{c})$ and one of $(\mathrm{d}-\mathrm{f})$ holds. If $(\mathrm{b})$ and $(\mathrm{e})$ hold, then $y \in R_{x}^{a}=D_{x}^{a}$, the second equality holding by (iv). If any other combination of (a-c) and (d-f) holds then, as explained in the previous paragraph, $x$ (and $y$ ) would belong to $P_{2}^{a}$ or $P_{3}^{a}$, a contradiction. This completes the proof that $J_{x}^{a} \subseteq D_{x}^{a}$. A dual argument shows that $J_{x}^{a}=D_{x}^{a}$ if $x \in P_{2}^{a} \backslash P_{3}^{a}$.

Finally, suppose $x \in P_{3}^{a}$. Let $z \in J_{x} \cap P_{3}^{a}$. So we have

$$
x=s^{\prime} a x a t^{\prime}, \quad z=s^{\prime \prime} a z a t^{\prime \prime}, \quad z=u^{\prime} x v^{\prime}, \quad x=u^{\prime \prime} z v^{\prime \prime} \quad \text { for some } s^{\prime}, s^{\prime \prime}, t^{\prime}, t^{\prime \prime}, u^{\prime}, u^{\prime \prime}, v^{\prime}, v^{\prime \prime} \in S^{(1)} .
$$

We then calculate $z=u^{\prime} x v^{\prime}=u^{\prime} s^{\prime} a x a t^{\prime} v^{\prime}=u^{\prime} s^{\prime} a\left(s^{\prime} a x a t^{\prime}\right) a t^{\prime} v^{\prime}=\left(u^{\prime} s^{\prime} a s^{\prime}\right) \star_{a} x \star_{a}\left(t^{\prime} a t^{\prime} v^{\prime}\right)$, and similarly $x=\left(u^{\prime \prime} s^{\prime \prime} a s^{\prime \prime}\right) \star_{a} z \star_{a}\left(t^{\prime \prime} a t^{\prime \prime} v^{\prime \prime}\right)$, showing that $z \mathscr{J}^{a} x$, and $J_{x} \cap P_{3}^{a} \subseteq J_{x}^{a}$. To prove the reverse inclusion, since we have already observed that $J_{x}^{a} \subseteq J_{x}$, it suffices to show that $J_{x}^{a} \subseteq P_{3}^{a}$. So suppose $y \in J_{x}^{a}$. If $y=x$, then $y \in P_{3}^{a}$, so suppose $y \neq x$. Then one of $(\mathrm{a}-\mathrm{c})$ and one of $(\mathrm{d}-\mathrm{f})$ above holds. If (a) and (d) hold, then

$$
y=\operatorname{sax}=\operatorname{sas}^{\prime} a x a t^{\prime}=\text { sas }^{\prime} \text { auayat },
$$

showing that $y \in P_{3}^{a}$. A similar argument covers the case in which (b) and (e) hold. As we observed above, any other combination of $(\mathrm{a}-\mathrm{c})$ and $(\mathrm{d}-\mathrm{f})$ implies that $y \in P_{3}^{a}$. This completes the proof.

For a pictorial understanding of Theorem 2.13, Figures 4 and 5 below give eggbox diagrams of various linear sandwich semigroups. Next, we show that stability of $S$ entails stability of all sandwich semigroups $S_{i j}^{a}$.

Proposition 2.14. Let $(S, \cdot, I, \lambda, \rho)$ be a stable partial semigroup. Then $S_{i j}^{a}$ is stable for all $i, j \in I$ and $a \in S_{j i}$.

Proof. Let $x, y \in S_{i j}$. We must show that

$$
x \mathscr{J}^{a} x \star_{a} y \Leftrightarrow x \mathscr{R}^{a} x \star_{a} y \quad \text { and } \quad x \mathscr{J}^{a} y \star_{a} x \Leftrightarrow x \mathscr{L}^{a} y \star_{a} x .
$$

By duality, it suffices to prove the first of these. Clearly, $x \mathscr{R}^{a} x \star_{a} y \Rightarrow x \mathscr{J}^{a} x \star_{a} y$. Conversely, suppose $x \mathscr{J}^{a} x \star_{a} y$. Then one of the following holds:
(i) $x=x a y$,
(iii) $x=$ uaxay for some $u \in S_{i j}$,
(ii) $x=$ xayav for some $v \in S_{i j}$,
(iv) $x=$ uaxayav for some $u, v \in S_{i j}$. 
Clearly, (i) or (ii) implies $x \mathscr{R}^{a} x a y$. Next, suppose (iv) holds. Then $x \mathscr{J}$ xaya, so that $x \mathscr{R} x a y a$ by stability. In particular, (a) $x=x a y a$ or (b) $x=x a y a w$ for some $w \in S_{i j}$. If (a) holds, then $x=$ (xaya)aya, so (b) holds with $w=a y a$. In particular, $x=\left(x \star_{a} y\right) \star_{a} w$, completing the proof that $x \mathscr{R}^{a} x \star_{a} y$. Finally, if (iii) holds, then $x=u a($ uaxay)ay, so that case (iii) reduces to case (iv). The proof is therefore complete.

We conclude this section with a result that shows how regularity of the sandwich element implies close relationships between certain sandwich semigroups $S_{i j}^{a}$ and $S_{j i}^{b}$ and certain (non-sandwich) subsemigroups of $S_{i}$ and $S_{j}$.

Theorem 2.15. Let $(S, \cdot, I, \lambda, \rho)$ be a partial semigroup and let $i, j \in I$. Let $a \in S_{j i}$ and $b \in S_{i j}$ be such that $a=a b a$ and $b=b a b$. Then

(i) $S_{i j}$ a and $a S_{i j}$ are subsemigroups of $S_{i}$ and $S_{j}$ (respectively),

(ii) $\left(a S_{i j} a, \star_{b}\right)$ and $\left(b S_{j i} b, \star_{a}\right)$ are monoids with identities $b$ and a (respectively), and are subsemigroups of $S_{j i}^{b}$ and $S_{i j}^{a}$ (respectively),

(iii) the maps $a S_{i j} a \rightarrow b S_{j i} b: x \mapsto b x b$ and $b S_{j i} b \rightarrow a S_{i j} a: x \mapsto$ axa define mutually inverse isomorphisms between $\left(a S_{i j} a, \star_{b}\right)$ and $\left(b S_{j i} b, \star_{a}\right)$,

(iv) $a \operatorname{Reg}\left(S_{i j}^{a}\right) a$ is contained in $\operatorname{Reg}\left(S_{j i}^{b}\right)$,

(v) the following diagrams commute, with all maps being homomorphisms:
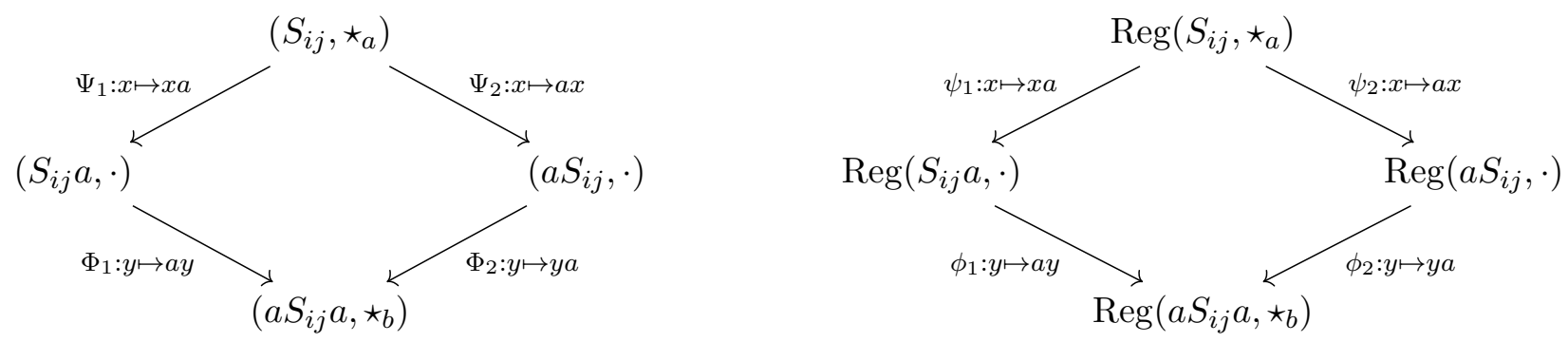

Proof. Part (i) is clear, and parts (ii) and (iii) are easily checked. Next, $\operatorname{suppose} x \in \operatorname{Reg}\left(S_{i j}^{a}\right)$, so $x=x a u a x$ for some $u \in S_{i j}$. Then axa $=$ axauaxa $=$ axabauabaxa $=(a x a) \star_{b}(a u a) \star_{b}(a x a)$, giving (iv). Part (v) is all mostly easy to check. That $\Phi_{1}$ is a homomorphism follows from $\Phi_{1}((x a)(y a))=$ axaya $=$ axabaya $=$ $\Phi_{1}(x a) \star_{b} \Phi_{1}(y a)$. It is clear that $\psi_{1} \operatorname{maps} \operatorname{Reg}\left(S_{i j}^{a}\right)$ into $\operatorname{Reg}\left(S_{i j} a\right)$. It follows from (iv) that $\phi_{1}$ maps $\operatorname{Reg}\left(S_{i j} a\right)$ into $\operatorname{Reg}\left(S_{j i}^{b}\right)$.

Remark 2.16. Other relationships exist, such as $\left(b a S_{i j} a, \cdot\right)=\left(b S_{j i} b a, \cdot\right)$, but these will not be explored any further.

\section{The linear partial semigroup}

As noted earlier, to understand the linear sandwich semigroups $\mathcal{M}_{m n}^{A}$, it is crucial to first understand the partial semigroup $\mathcal{M}$. So in this section, we gather the required material on $\mathcal{M}$, showing how the general framework of Section 2 applies in this case.

We fix a field $\mathbb{F}$ for the remainder of the article. For positive integers $m, n \in \mathbb{N}=\{1,2,3, \ldots\}$, we write $\mathcal{M}_{m n}=\mathcal{M}_{m n}(\mathbb{F})$ for the set of all $m \times n$ matrices (i.e., all matrices with $m$ rows and $n$ columns) over $\mathbb{F}$. We write $\mathcal{M}=\mathcal{M}(\mathbb{F})=\bigcup_{m, n \in \mathbb{N}} \mathcal{M}_{m n}$ for the set of all (finite dimensional, non-empty) matrices over $\mathbb{F}$. So $\mathcal{M}$ is a partial semigroup, as noted in Example 2.5. By convention, we consider there to be a unique $m \times 0$ and $0 \times n$ matrix for any $m, n \geq 0$, namely the empty matrix, which we denote by $\emptyset$. So $\mathcal{M}_{m n}=\{\emptyset\}$ if $m=0$ or $n=0$. But this is a matter of convenience, and we do not consider the empty matrix $\emptyset$ to be an element of $\mathcal{M}$. We also write $\mathcal{M}_{n}=\mathcal{M}_{n}(\mathbb{F})=\mathcal{M}_{n n}$ for any $n$, and denote by $\mathcal{G}_{n}=\mathcal{G}_{n}(\mathbb{F})$ the group of $n \times n$ 
invertible matrices over $\mathbb{F}$. So $\mathcal{M}_{n}$ and $\mathcal{G}_{n}$ are the full linear monoid and general linear group of degree $n$. For background on the full linear monoids, the monograph [74] is highly recommended.

If $V$ and $W$ are vector spaces, we write $\operatorname{Hom}(V, W)$ for the set of all linear transformations from $V$ to $W$. As usual, if $\alpha \in \operatorname{Hom}(V, W)$, we write $\operatorname{im}(\alpha)=\{\alpha(v): v \in V\}$ and $\operatorname{ker}(\alpha)=\{v \in V: \alpha(v)=0\}$ for the image and kernel of $\alpha$. We write $\operatorname{End}(V)=\operatorname{Hom}(V, V)$ for the monoid of all endomorphisms of $V$ (i.e., all linear transformations $V \rightarrow V$ ), and $\operatorname{Aut}(V)$ for the group of all automorphisms of $V$ (i.e., all invertible endomorphisms of $V)$. For $n \geq 0$, we write $V_{n}=\mathbb{F}^{n}$ for the vector space of all $n \times 1$ column vectors over $\mathbb{F}$. We will identify $\mathcal{M}_{m n}$ with $\operatorname{Hom}\left(V_{n}, V_{m}\right)$ in the usual way. Namely, if $X \in \mathcal{M}_{m n}$, we write $\lambda_{X} \in \operatorname{Hom}\left(V_{n}, V_{m}\right)$ for the linear transformation $\lambda_{X}: V_{n} \rightarrow V_{m}$ defined by $\lambda_{X}(v)=X v$ for all $v \in V_{n}$. We will often prove statements about $\mathcal{M}_{m n}$ by proving the equivalent statement about $\operatorname{Hom}\left(V_{n}, V_{m}\right)$. When $m=n$, the map $X \rightarrow \lambda_{X}$ determines an isomorphism of monoids $\mathcal{M}_{n} \rightarrow \operatorname{End}\left(V_{n}\right)$, and its restriction to $\mathcal{G}_{n} \subseteq \mathcal{M}_{n}$ determines an isomorphism of groups $\mathcal{G}_{n} \rightarrow \operatorname{Aut}\left(V_{n}\right)$. We write $\left\{e_{n 1}, \ldots, e_{n n}\right\}$ for the standard basis of $V_{n}\left(e_{n i}\right.$ has a 1 in position $i$ and 0 's elsewhere). We also write $W_{n s}=\operatorname{span}\left\{e_{n 1}, \ldots, e_{n s}\right\}$ for each $0 \leq s \leq n$. (We interpret $\operatorname{span} \emptyset=\{0\}$, though the dimension of the ambient space must be understood from context.)

Our first aim is to characterise Green's relations $(\mathscr{R}, \mathscr{L}, \mathscr{J}, \mathscr{D}, \mathscr{H})$ and preorders $(\leq \mathscr{R}, \leq \mathscr{L}, \leq \mathscr{J})$ on $\mathcal{M}$. Because $\mathcal{M}$ is monoidal (see Definition 2.1), $\mathcal{M}=\mathcal{M}^{(1)}$. So, for example, if $X, Y \in \mathcal{M}$ are two matrices (not necessarily of the same size), then $X \leq \mathscr{R} Y$ if and only if $X=Y A$ for some $A \in \mathcal{M}$. Note that if $X \leq_{\mathscr{R}} Y$ (resp., $X \leq_{\mathscr{L}} Y$ ), then $X$ and $Y$ must have the same number of rows (resp., columns).

Let $X \in \mathcal{M}_{m n}$. For $1 \leq i \leq m$ and $1 \leq j \leq n$, we write $\mathfrak{r}_{i}(X)$ and $\mathfrak{c}_{j}(X)$ for the $i$ th row and $j$ th column of $X$, respectively. We write $\operatorname{Row}(X)=\operatorname{span}\left\{\mathfrak{r}_{1}(X), \ldots, \mathfrak{r}_{m}(X)\right\}$ and $\operatorname{Col}(X)=\operatorname{span}\left\{\mathfrak{c}_{1}(X), \ldots, \mathfrak{c}_{n}(X)\right\}$ for the row space and column space of $X$, respectively, and we write $\operatorname{rank}(X)=\operatorname{dim}(\operatorname{Row}(X))=\operatorname{dim}(\operatorname{Col}(X))$ for the rank of $X$. Because of the transpose map $\mathcal{M} \rightarrow \mathcal{M}: A \mapsto A^{\mathrm{T}}$, which is a bijection and satisfies $(A B)^{\mathrm{T}}=B^{\mathrm{T}} A^{\mathrm{T}}$, the linear partial semigroup $\mathcal{M}$ is self-dual (in the sense that it is anti-isomorphic to its own dual). Since $\operatorname{Row}\left(X^{\mathrm{T}}\right)=\operatorname{Col}(X)$, any statement about row spaces implies a corresponding dual statement about column spaces (and vice versa). (Without causing confusion, we will often blur the distinction between row vectors and column vectors, and think of $\operatorname{Row}(X)$ and $\operatorname{Col}(X)$ as subspaces of $\mathbb{F}^{n}$ and $\mathbb{F}^{m}$, respectively.)

The next result characterises Green's relations and preorders on $\mathcal{M}$ in terms of the parameters introduced above. An equivalent formulation in the special case of square matrices may be found in 74, Lemma 2.1].

Lemma 3.1. Let $X, Y \in \mathcal{M}$. Then

(i) $X \leq_{\mathscr{R}} Y \Leftrightarrow \operatorname{Col}(X) \subseteq \operatorname{Col}(Y)$,

(ii) $X \leq \mathscr{L} Y \Leftrightarrow \operatorname{Row}(X) \subseteq \operatorname{Row}(Y)$,

(iii) $X \leq \mathscr{J} Y \Leftrightarrow \operatorname{rank}(X) \subseteq \operatorname{rank}(Y)$, (iv) $X \mathscr{R} Y \Leftrightarrow \operatorname{Col}(X)=\operatorname{Col}(Y)$,

(v) $X \mathscr{L} Y \Leftrightarrow \operatorname{Row}(X)=\operatorname{Row}(Y)$,

(vi) $X \mathscr{J} Y \Leftrightarrow \operatorname{rank}(X)=\operatorname{rank}(Y)$.

BFurther, $\mathcal{M}$ is stable, so $\mathscr{J}=\mathscr{D}$.

Proof. Clearly, (iv-vi) follow from (i-iii). Note that (ii) is the dual of (i), which is true because

$$
\begin{aligned}
X \leq_{\mathscr{R}} Y & \Leftrightarrow X=Y A \text { for some } A \in \mathcal{M} \\
& \Leftrightarrow \text { every column of } X \text { is a linear combination of the columns of } Y \\
& \Leftrightarrow \operatorname{Col}(X) \subseteq \operatorname{Col}(Y) .
\end{aligned}
$$

For (iii), if $X \leq \mathscr{J} Y$, then $X=A Y B$ for some $A, B \in \mathcal{M}$, giving $\operatorname{rank}(X)=\operatorname{rank}(A Y B) \leq \operatorname{rank}(Y)$. Conversely, suppose $\operatorname{rank}(X) \leq \operatorname{rank}(Y)$, and say $X \in \mathcal{M}_{m n}$ and $Y \in \mathcal{M}_{k l}$. It is sufficient to show that $\lambda_{X}=\alpha \circ \lambda_{Y} \circ \beta$ for some $\alpha \in \operatorname{Hom}\left(V_{k}, V_{m}\right)$ and $\beta \in \operatorname{Hom}\left(V_{n}, V_{l}\right)$. Put $r=\operatorname{rank}(X)$ and $s=$ $\operatorname{rank}(Y)$. Choose bases $\mathscr{B}_{1}=\left\{u_{1}, \ldots, u_{n}\right\}$ and $\mathscr{B}_{2}=\left\{v_{1}, \ldots, v_{l}\right\}$ for $V_{n}$ and $V_{l}$ so that $\left\{u_{r+1}, \ldots, u_{n}\right\}$ and $\left\{v_{s+1}, \ldots, v_{l}\right\}$ are bases for $\operatorname{ker}\left(\lambda_{X}\right)$ and $\operatorname{ker}\left(\lambda_{Y}\right)$, respectively. Extend (if necessary) the linearly independent sets $\left\{\lambda_{Y}\left(v_{1}\right), \ldots, \lambda_{Y}\left(v_{r}\right)\right\}$ and $\left\{\lambda_{X}\left(u_{1}, \ldots, \lambda_{X}\left(u_{r}\right)\right\}\right.$ arbitrarily to bases

$$
\mathscr{B}_{3}=\left\{\lambda_{Y}\left(v_{1}\right), \ldots, \lambda_{Y}\left(v_{r}\right), w_{r+1}, \ldots, w_{k}\right\} \quad \text { and } \quad \mathscr{B}_{4}=\left\{\lambda_{X}\left(u_{1}\right), \ldots, \lambda_{X}\left(u_{r}\right), x_{r+1}, \ldots, x_{m}\right\}
$$


for $V_{k}$ and $V_{m}$. Now let $\alpha \in \operatorname{Hom}\left(V_{k}, V_{m}\right)$ and $\beta \in \operatorname{Hom}\left(V_{n}, V_{l}\right)$ be chosen arbitrarily so that

$$
\begin{aligned}
\alpha\left(\lambda_{Y}\left(v_{i}\right)\right) & =\lambda_{X}\left(u_{i}\right), & \alpha\left(w_{j}\right) & \in \operatorname{span}\left\{x_{r+1}, \ldots, x_{m}\right\} \\
\beta\left(u_{i}\right) & =v_{i}, & & \text { for all } 1 \leq i \leq r \text { and } r+1 \leq j \leq k, \\
\left(u_{j}\right) & \in \operatorname{span}\left\{v_{s+1}, \ldots, v_{l}\right\} & & \text { for all } 1 \leq i \leq r \text { and } r+1 \leq j \leq n .
\end{aligned}
$$

One easily checks that $\alpha \circ \lambda_{Y} \circ \beta=\lambda_{X}$, by checking the respective actions on the basis $\mathscr{B}_{1}$ of $V_{n}$.

To prove stability, we must show that for all $X, Y \in \mathcal{M}$,

$$
X \mathscr{J} X Y \Leftrightarrow X \mathscr{R} X Y \quad \text { and } \quad X \mathscr{J} Y X \Leftrightarrow X \mathscr{L} Y X .
$$

By duality, it suffices to prove the first equivalence. Since $\mathscr{R} \subseteq \mathscr{J}$, it is enough to prove that $X \mathscr{J} X Y \Rightarrow$ $X \mathscr{R} X Y$. Now, $\operatorname{Col}(X Y) \subseteq \operatorname{Col}(X)$. But also $X \mathscr{J} X Y$ gives $\operatorname{dim}(\operatorname{Col}(X))=\operatorname{rank}(X)=\operatorname{rank}(X Y)=$ $\operatorname{dim}(\operatorname{Col}(X Y))$, so that $\operatorname{Col}(X)=\operatorname{Col}(X Y)$, whence $X \mathscr{R} X Y$.

As we saw in Section 2, stability and regularity are very useful properties for a partial semigroup to have. Now that we know $\mathcal{M}$ is stable, let us show that $\mathcal{M}$ is also regular.

Lemma 3.2. The linear partial semigroup $\mathcal{M}$ is regular.

Proof. Let $X \in \mathcal{M}_{m n}$. It suffices to show that there exists $\alpha \in \operatorname{Hom}\left(V_{m}, V_{n}\right)$ such that $\lambda_{X}=\lambda_{X} \circ \alpha \circ \lambda_{X}$. Let $\mathscr{B}=\left\{v_{1}, \ldots, v_{n}\right\}$ be a basis of $V_{n}$ such that $\left\{v_{r+1}, \ldots, v_{n}\right\}$ is a basis of $\operatorname{ker}\left(\lambda_{X}\right)$. Extend (if necessary) the linearly independent set $\left\{\lambda_{X}\left(v_{1}\right), \ldots, \lambda_{X}\left(v_{r}\right)\right\}$ to a basis $\left\{\lambda_{X}\left(v_{1}\right), \ldots, \lambda_{X}\left(v_{r}\right), w_{r+1}, \ldots, w_{m}\right\}$ of $V_{m}$. Let $\alpha \in \operatorname{Hom}\left(V_{m}, V_{n}\right)$ be any linear transformation for which $\alpha\left(\lambda_{X}\left(v_{i}\right)\right)=v_{i}$ for each $1 \leq i \leq r$. Then one easily checks that $\lambda_{X}=\lambda_{X} \circ \alpha \circ \lambda_{X}$ by calculating the action on the basis $\mathscr{B}$.

As in Section 2, if $X \in \mathcal{M}_{m n}$ and $\mathscr{K}$ is one of $\mathscr{R}, \mathscr{L}, \mathscr{J}, \mathscr{D}, \mathscr{H}$, we write $K_{X}=\left\{Y \in \mathcal{M}_{m n}: X \mathscr{K} Y\right\}$, and call $K_{X}$ the $\mathscr{K}$-class of $X$ in $\mathcal{M}_{m n}$. Note that all matrices from $K_{X}$ have the same dimensions. (We will have no need to consider the sets $[X]_{\mathscr{K}}$ of all matrices $\mathscr{K}$-related to $X$.) Recall that $\mathcal{G}_{k}$ denotes the group of all invertible $k \times k$ matrices over $\mathbb{F}$. The next result gives an alternative description of various Green's classes in $\mathcal{M}$.

Lemma 3.3. Let $X \in \mathcal{M}_{m n}$. Then

(i) $R_{X}=\left\{Y \in \mathcal{M}_{m n}: \operatorname{Col}(X)=\operatorname{Col}(Y)\right\}=X \mathcal{G}_{n}$,

(ii) $L_{X}=\left\{Y \in \mathcal{M}_{m n}: \operatorname{Row}(X)=\operatorname{Row}(Y)\right\}=\mathcal{G}_{m} X$,

(iii) $D_{X}=J_{X}=\left\{Y \in \mathcal{M}_{m n}: \operatorname{rank}(X)=\operatorname{rank}(Y)\right\}=\mathcal{G}_{m} X \mathcal{G}_{n}$.

Proof. For (i), note that clearly $X \mathcal{G}_{n} \subseteq R_{X}$. By Lemma 3.1, it remains to show the reverse inclusion, so suppose $Y \in R_{X}$. In particular, $X \mathscr{J} Y$, so $\operatorname{rank}(X)=\operatorname{rank}(Y)$. Put $r=\operatorname{rank}(X)$. We show that $\lambda_{Y}=\lambda_{X} \circ \alpha$ for some $\alpha \in \operatorname{Aut}\left(V_{n}\right)$. Since $X \mathscr{R} Y$, we already know that $\lambda_{Y}=\lambda_{X} \circ \beta$ for some $\beta \in$ $\operatorname{End}\left(V_{n}\right)$. Let $\mathscr{B}_{1}=\left\{u_{1}, \ldots, u_{n}\right\}$ be a basis of $V_{n}$ such that $\left\{u_{r+1}, \ldots, u_{n}\right\}$ is a basis of $\operatorname{ker}\left(\lambda_{Y}\right)$. So $\left\{\lambda_{X}\left(\beta\left(u_{1}\right)\right), \ldots, \lambda_{X}\left(\beta\left(u_{r}\right)\right)\right\}=\left\{\lambda_{Y}\left(u_{1}\right), \ldots, \lambda_{Y}\left(u_{r}\right)\right\}$ is a basis of $\operatorname{im}\left(\lambda_{Y}\right)$. It follows that $\left\{\beta\left(u_{1}\right), \ldots, \beta\left(u_{r}\right)\right\}$ is linearly independent. We may therefore extend this set to a basis $\mathscr{B}_{2}=\left\{\beta\left(u_{1}\right), \ldots, \beta\left(u_{r}\right), v_{r+1}, \ldots, v_{n}\right\}$ of $V_{n}$, where $\left\{v_{r+1}, \ldots, v_{n}\right\}$ is a basis of $\operatorname{ker}\left(\lambda_{X}\right)$. Now define $\alpha \in \operatorname{Aut}\left(V_{n}\right)$ by

$$
\alpha\left(u_{i}\right)= \begin{cases}\beta\left(u_{i}\right) & \text { if } 1 \leq i \leq r \\ v_{i} & \text { if } r<i \leq n .\end{cases}
$$

One easily checks that $\lambda_{Y}=\lambda_{X} \circ \alpha$. This completes the proof of (i).

Part (ii) is dual to (i). For (iii), clearly $\mathcal{G}_{m} X \mathcal{G}_{n} \subseteq J_{X}$, and the converse follows quickly from (i) and (ii) and the fact that $\mathscr{J}=\mathscr{D}=\mathscr{L} \circ \mathscr{R}$. By Lemma 3.1. this completes the proof.

If $\mathscr{K}$ is one of $\mathscr{R}, \mathscr{L}, \mathscr{D}=\mathscr{J}$, then the set $\mathcal{M}_{m n} / \mathscr{K}$ of all $\mathscr{K}$-classes of $\mathcal{M}_{m n}$ inherits a partial order:

$$
K_{X} \leq \mathscr{K} K_{Y} \Leftrightarrow X \leq \mathscr{K} Y .
$$


We typically write $\leq$ for the order $\leq \mathscr{J}$ on the $\mathscr{D}=\mathscr{J}$-classes. Of importance is the fact that these classes form a chain:

$$
D_{0}\left(\mathcal{M}_{m n}\right)<D_{1}\left(\mathcal{M}_{m n}\right)<\cdots<D_{l}\left(\mathcal{M}_{m n}\right),
$$

where $D_{s}\left(\mathcal{M}_{m n}\right)=\left\{X \in \mathcal{M}_{m n}: \operatorname{rank}(X)=s\right\}$ for all $0 \leq s \leq l=\min (m, n)$.

Figure 2 pictures an eggbox diagram (as explained in Section 2 of the $\mathscr{D}$-class $D_{1}\left(\mathcal{M}_{23}\left(\mathbb{Z}_{3}\right)\right.$ ) of all $2 \times 3$ matrices of rank 1 over the field $\mathbb{F}=\mathbb{Z}_{3}=\{0,1,2\}$ (see Lemma 3.4 for an explanation of the number and sizes of the $\mathscr{R}$-, $\mathscr{L}$ - and $\mathscr{H}$-classes). The reader need not yet worry about the subdivisions within the eggbox; for now, it is enough to note that the matrices to the left (resp., top) of the vertical (resp., horizontal) divider satisfy the property that the first column (resp., row) spans the column space (resp., row space) of the matrix.

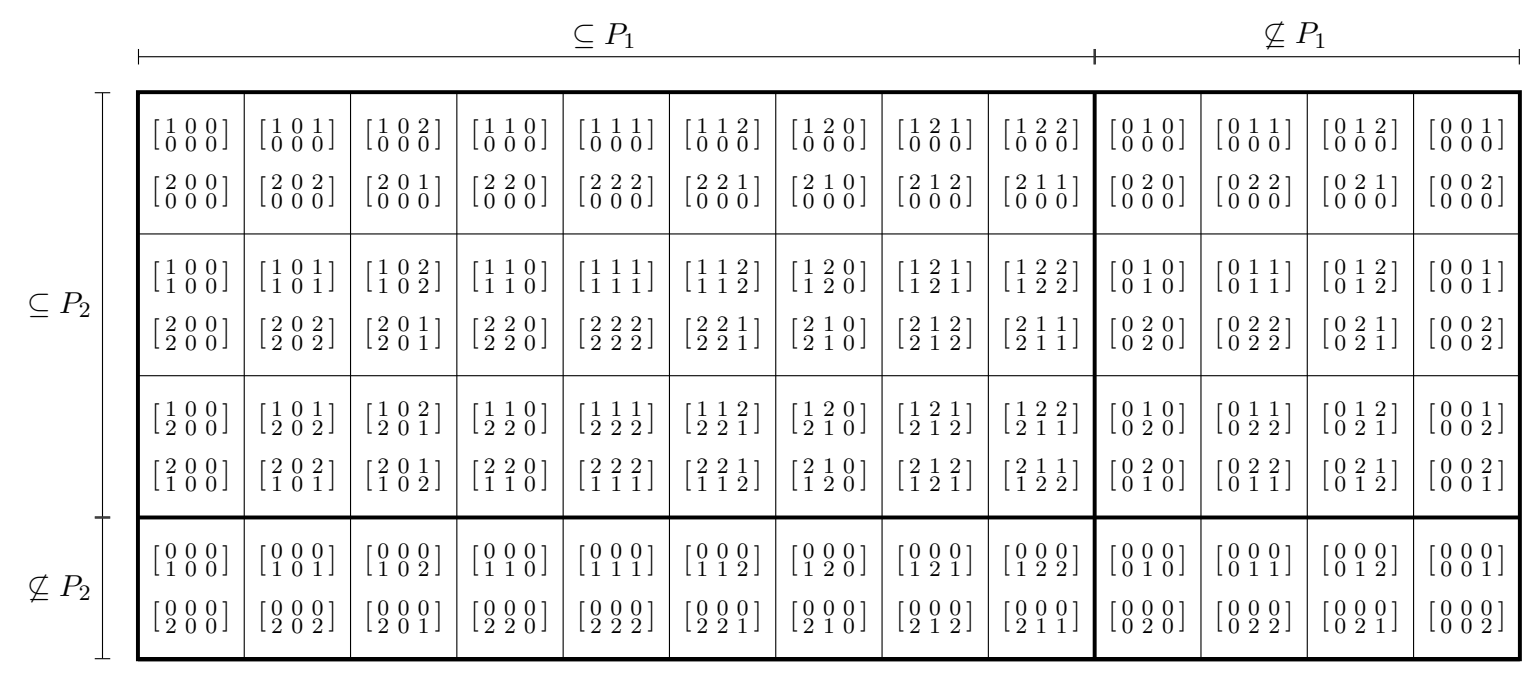

Figure 2: An eggbox diagram of the $\mathscr{D}$-class $D_{1}\left(\mathcal{M}_{23}\left(\mathbb{Z}_{3}\right)\right)$.

So $\mathcal{M}_{m n}$ has $\min (m, n)+1 \mathscr{D}$-classes. It will also be convenient to have some more combinatorial information about the number and size of certain $\mathscr{K}$-classes. Recall that the $q$-factorials and $q$-binomial coefficients are defined by

$$
[s]_{q} !=1 \cdot(1+q) \cdots\left(1+q+\cdots+q^{s-1}\right)=\frac{(q-1)\left(q^{2}-1\right) \cdots\left(q^{s}-1\right)}{(q-1)^{s}}
$$

and

$$
\left[\begin{array}{c}
m \\
s
\end{array}\right]_{q}=\frac{[m]_{q} !}{[s]_{q} ![m-s]_{q} !}=\frac{\left(q^{m}-1\right)\left(q^{m}-q\right) \cdots\left(q^{m}-q^{s-1}\right)}{\left(q^{s}-1\right)\left(q^{s}-q\right) \cdots\left(q^{s}-q^{s-1}\right)}=\frac{\left(q^{m}-1\right)\left(q^{m-1}-1\right) \cdots\left(q^{m-s+1}-1\right)}{\left(q^{s}-1\right)\left(q^{s-1}-1\right) \cdots(q-1)} .
$$

It is easy to check (and well-known) that when $|\mathbb{F}|=q<\infty$,

$$
\left|\mathcal{G}_{s}\right|=\left(q^{s}-1\right)\left(q^{s}-q\right) \cdots\left(q^{s}-q^{s-1}\right)=q^{\left(\begin{array}{c}
s \\
2
\end{array}\right)}(q-1)^{s}[s]_{q} !
$$

In what follows, a crucial role will be played by the matrices $J_{m n s} \in \mathcal{M}_{m n}$ defined for $s \leq \min (m, n)$ by

$$
J_{m n s}=\left[\begin{array}{cc}
I_{s} & O_{s, n-s} \\
O_{m-s, s} & O_{m-s, n-s}
\end{array}\right] .
$$

Here and elsewhere, we write $I_{s}$ and $O_{k l}$ for the $s \times s$ identity matrix and $k \times l$ zero matrix (respectively). If the dimensions are understood from context, we just write $O=O_{k l}$. So $J_{m n s}$ is the $m \times n$ matrix with 1's in the first $s$ positions on the leading diagonal and 0's elsewhere. Note that if $s=m \leq n$ (resp., $s=n \leq m$ ), then the matrices $O_{m-s, s}$ and $O_{m-s, n-s}$ (resp., $O_{s, n-s}$ and $\left.O_{m-s, n-s}\right)$ are empty, and $J_{m n s}=\left[I_{s} O_{s, n-s}\right]$ (resp., $\left.\left[\begin{array}{c}I_{s} \\ O_{m-s, s}\end{array}\right]\right)$.

Lemma 3.4. Suppose $|\mathbb{F}|=q<\infty$, and let $0 \leq s \leq \min (m, n)$. Then

(i) $D_{s}\left(\mathcal{M}_{m n}\right)$ contains $\left[\begin{array}{c}m \\ s\end{array}\right]_{q} \mathscr{R}$-classes, 
(ii) $D_{s}\left(\mathcal{M}_{m n}\right)$ contains $\left[\begin{array}{l}n \\ s\end{array}\right]_{q} \mathscr{L}$-classes,

(iii) $D_{s}\left(\mathcal{M}_{m n}\right)$ contains $\left[\begin{array}{c}m \\ s\end{array}\right]_{q}\left[\begin{array}{l}n \\ s\end{array}\right]_{q} \mathscr{H}$-classes, each of which has size $\left|\mathcal{G}_{s}\right|=q^{\left(\begin{array}{c}s \\ 2\end{array}\right)}(q-1)^{s}[s]_{q}$ !,

(iv) $\left|D_{s}\left(\mathcal{M}_{m n}\right)\right|=\left[\begin{array}{c}m \\ s\end{array}\right]_{q}\left[\begin{array}{c}n \\ s\end{array}\right]_{q} q^{\left(\begin{array}{c}s \\ 2\end{array}\right)}(q-1)^{s}[s]_{q} !$

Proof. Parts (i) and (ii) follow immediately from parts (i) and (ii) of Lemma 3.3 and the well-known fact that $\left[\begin{array}{c}m \\ s\end{array}\right]_{q}$ is the number of $s$ dimensional subspaces of an $m$ dimensional vector space over $\mathbb{F}$. The number of $\mathscr{H}$-classes follows immediately from (i) and (ii). By Lemma 2.8, all the $\mathscr{H}$-classes in $D_{s}\left(\mathcal{M}_{m n}\right)$ have the same size, so it suffices to calculate the size of $H=H_{J_{m n s}}$. Let $X=\left[{ }_{C}^{A} \underset{D}{B}\right] \in H$, where $A \in \mathcal{M}_{s}, B \in \mathcal{M}_{s, n-s}$, and so on. Since $\operatorname{Row}(X)=\operatorname{Row}\left(J_{m n s}\right)$, we see that $B$ and $D$ are zero matrices. Considering column spaces, we see that $C$ is also a zero matrix. It follows that $X=\left[\begin{array}{ll}A & O \\ O & O\end{array}\right]$, and also $\operatorname{rank}(A)=\operatorname{rank}(X)=\operatorname{rank}\left(J_{m n s}\right)=s$. Clearly every such matrix $X=\left[\begin{array}{cc}A & O \\ O & O\end{array}\right]$ with $\operatorname{rank}(A)=s$ belongs to $H$. The condition that $\operatorname{rank}(A)=s$ is equivalent to $A \in \mathcal{G}_{s}$, so it follows that $|H|=\left|\mathcal{G}_{s}\right|$. Finally, (iv) follows from (iii).

Of course, by considering the size of $\mathcal{M}_{m n}$ when $|\mathbb{F}|=q<\infty$, we obtain the identity

$$
q^{m n}=\sum_{s=0}^{l}\left[\begin{array}{c}
m \\
s
\end{array}\right]_{q}\left[\begin{array}{l}
n \\
s
\end{array}\right]_{q}[s]_{q} !(q-1)^{s} q^{\left(\begin{array}{c}
s \\
2
\end{array}\right)} .
$$

We conclude this section by stating some well-known results on the full linear monoids $\mathcal{M}_{n}$ and their ideals that we will require in what follows. The set $E\left(\mathcal{M}_{n}\right)=\left\{X \in \mathcal{M}_{n}: X=X^{2}\right\}$ of idempotents of $\mathcal{M}_{n}$ is not a subsemigroup (unless $n \leq 1$ ), but the subsemigroup $\mathcal{E}_{n}=\left\langle E\left(\mathcal{M}_{n}\right)\right\rangle$ of $\mathcal{M}_{n}$ generated by these idempotents has a neat description. Namely, it was shown by Erdos [25] that any singular (i.e., non-invertible) matrix over $\mathbb{F}$ is a product of idempotent matrices. This result has been reproved by a number of authors 2, 15, 18, 27, 53,. The minimal number of (idempotent) matrices required to generate $\mathcal{E}_{n}$ was determined by Dawlings [16]. Recall that the rank (resp., idempotent rank) of a semigroup (resp., idempotent generated semigroup) $S$, denoted $\operatorname{rank}(S)(\operatorname{resp} ., \operatorname{idrank}(S))$, is the minimal size of a generating set (resp., idempotent generating set) for $S$. (The rank of a semigroup should not be confused with the rank of a matix.) If $U$ is a subset of a semigroup $S$, we write $E(U)=E(S) \cap U$ for the set of all idempotents from $U$.

Theorem 3.5 (Erdos [25], Dawlings [15, 16]). We have

$$
\mathcal{E}_{n}=\left\langle E\left(\mathcal{M}_{n}\right)\right\rangle=\left(\mathcal{M}_{n} \backslash \mathcal{G}_{n}\right) \cup\left\{I_{n}\right\} \quad \text { and } \quad \mathcal{M}_{n} \backslash \mathcal{G}_{n}=\left\langle E\left(D_{n-1}\left(\mathcal{M}_{n}\right)\right)\right\rangle .
$$

Further, if $|\mathbb{F}|=q<\infty$, then

$$
\operatorname{rank}\left(\mathcal{M}_{n} \backslash \mathcal{G}_{n}\right)=\operatorname{idrank}\left(\mathcal{M}_{n} \backslash \mathcal{G}_{n}\right)=\left(q^{n}-1\right) /(q-1) .
$$

The previous result has been extended by Gray [33] to arbitrary ideals of $\mathcal{M}_{n}$.

Theorem 3.6 (Gray [33]). The ideals of $\mathcal{M}_{n}$ are precisely the sets

$$
I_{s}\left(\mathcal{M}_{n}\right)=D_{0}\left(\mathcal{M}_{n}\right) \cup \cdots \cup D_{s}\left(\mathcal{M}_{n}\right)=\left\{X \in \mathcal{M}_{n}: \operatorname{rank}(X) \leq s\right\} \quad \text { for } 0 \leq s \leq n,
$$

and they form a chain: $I_{0}\left(\mathcal{M}_{n}\right) \subseteq \cdots \subseteq I_{n}\left(\mathcal{M}_{n}\right)$. If $0 \leq s<n$, then $I_{s}\left(\mathcal{M}_{n}\right)=\left\langle E\left(D_{s}\left(\mathcal{M}_{n}\right)\right)\right\rangle$ is generated by the idempotents in its top $\mathscr{D}$-class. Further, if $|\mathbb{F}|=q<\infty$, then

$$
\operatorname{rank}\left(I_{s}\left(\mathcal{M}_{n}\right)\right)=\operatorname{idrank}\left(I_{s}\left(\mathcal{M}_{n}\right)\right)=\left[\begin{array}{l}
n \\
s
\end{array}\right]_{q} .
$$

Note that $I_{n}\left(\mathcal{M}_{n}\right)=\mathcal{M}_{n}, D_{n}\left(\mathcal{M}_{n}\right)=\mathcal{G}_{n}$ and $I_{n-1}\left(\mathcal{M}_{n}\right)=\mathcal{M}_{n} \backslash \mathcal{G}_{n}$, so Theorem 3.5 is a special case of Theorem 3.6 since $\left[\begin{array}{c}n \\ n-1\end{array}\right]_{q}=\left(q^{n}-1\right) /(q-1)$.

On several occasions, we will need to make use of the fact that the general linear group $\mathcal{G}_{n}$ may be generated by two matrices, as was originally proved by Waterhouse [94]; see also [31], where minimal generating sets for $\mathcal{G}_{n}$ are explored in more detail. Probabilistic generation of matrix groups is considered in [4, 36], for example, though the context is usually for classical groups. 
Theorem 3.7 (Waterhouse 94$]$ ). If $|\mathbb{F}|<\infty$, then

(i) $\operatorname{rank}\left(\mathcal{G}_{1}\right)=1$, and $\operatorname{rank}\left(\mathcal{G}_{n}\right)=2$ if $n \geq 2$,

(ii) $\mathcal{M}_{n}=\left\langle\mathcal{G}_{n} \cup\{X\}\right\rangle$ for any $X \in D_{n-1}\left(\mathcal{M}_{n}\right)$,

(iii) $\operatorname{rank}\left(\mathcal{M}_{1}\right)=2$, and $\operatorname{rank}\left(\mathcal{M}_{n}\right)=3$ if $n \geq 2$.

For convenience, eggbox diagrams are given for the full linear monoids $\mathcal{M}_{n}\left(\mathbb{Z}_{2}\right)$ for $0 \leq n \leq 3$ in Figure 3 below. In the diagrams, group $\mathscr{H}$-classes are shaded grey, and a label of $\mathrm{k}$ indicates that the group $\mathscr{H}$-class is isomorphic to $\mathcal{G}_{k}\left(\mathbb{Z}_{2}\right)$.
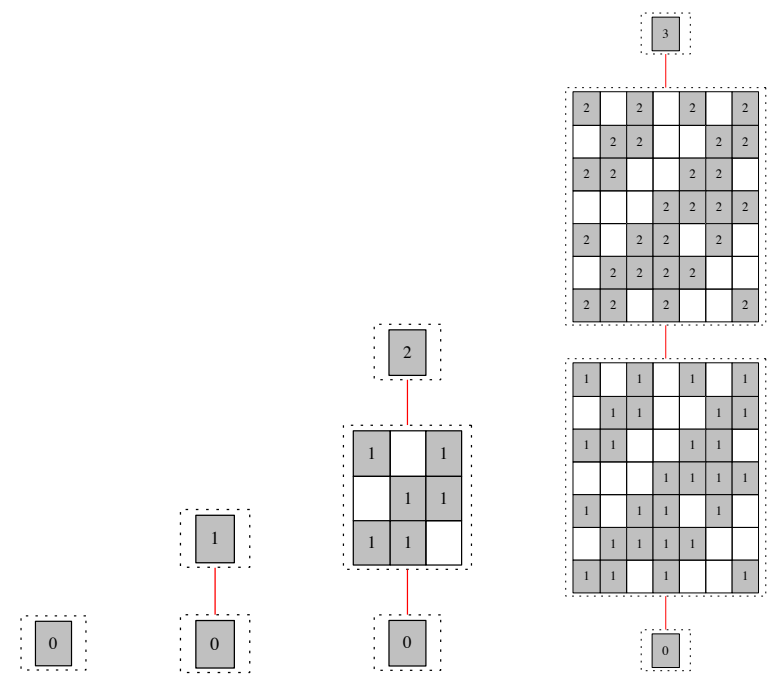

Figure 3: Egg box diagrams of the full linear semigroups $\mathcal{M}_{0}, \mathcal{M}_{1}, \mathcal{M}_{2}, \mathcal{M}_{3}$, all over $\mathbb{Z}_{2}$ (left to right).

\section{Linear sandwich semigroups}

Now that we have gathered the required material on $\mathcal{M}$, we may begin our study of the linear sandwich semigroups. From now on, we fix integers $m, n \geq 1$ and an $n \times m$ matrix $A \in \mathcal{M}_{n m}$. As in Section 2, we denote by

$$
\mathcal{M}_{m n}^{A}=\mathcal{M}_{m n}^{A}(\mathbb{F})=\left(\mathcal{M}_{m n}, \star_{A}\right)
$$

the sandwich semigroup of $\mathcal{M}_{m n}$ under the operation $\star_{A}$ defined by

$$
X \star_{A} Y=X A Y \quad \text { for } X, Y \in \mathcal{M}_{m n} .
$$

We note that if $m=n$, then $\mathcal{M}_{m n}^{A}=\mathcal{M}_{n}^{A}$ is a variant 38 of the full linear monoid $\mathcal{M}_{n}$, so everything we prove about linear sandwich semigroups holds for such linear variants also. We begin with a simple observation.

Lemma 4.1. (i) If $A \in \mathcal{M}_{n m}$, then $\mathcal{M}_{m n}^{A} \cong \mathcal{M}_{n m}^{A^{\mathrm{T}}}$.

(ii) If $A, B \in \mathcal{M}_{n m}$ are such that $\operatorname{rank}(A)=\operatorname{rank}(B)$, then $\mathcal{M}_{m n}^{A} \cong \mathcal{M}_{m n}^{B}$.

Proof. It clear that $X \mapsto X^{\mathrm{T}}$ defines an isomorphism $\mathcal{M}_{m n}^{A} \rightarrow \mathcal{M}_{n m}^{A^{T}}$, giving (i). Next, if $\operatorname{rank}(A)=$ $\operatorname{rank}(B)$, Lemma 3.3 gives $A=U B V$ for some $U \in \mathcal{G}_{m}$ and $V \in \mathcal{G}_{n}$. But then one may check that $X \mapsto V X U$ defines an isomorphism $\mathcal{M}_{m n}^{A} \rightarrow \mathcal{M}_{m n}^{B}$, giving (ii).

In particular, when studying the semigroup $\mathcal{M}_{m n}^{A}$ where $\operatorname{rank}(A)=r$, we may choose any $A \in \mathcal{M}_{n m}$ of rank $r$. For the rest of the article, we will therefore study the semigroup $\mathcal{M}_{m n}^{J}$, where

$$
J=J_{n m r}=\left[\begin{array}{cc}
I_{r} & O_{r, m-r} \\
O_{n-r, r} & O_{n-r, m-r}
\end{array}\right] \in \mathcal{M}_{n m} .
$$


From now on, unless otherwise specified, whenever a $k \times l$ matrix $X$ (with $k, l \in\{m, n\}$ ) is written in $2 \times 2$ block form, $X=\left[\begin{array}{ll}A & B \\ C & D\end{array}\right]$, we will be tacitly assuming that $A \in \mathcal{M}_{r}$ (from which the dimensions of $B, C, D$ may be deduced). So for example, we will usually just write $J=\left[\begin{array}{cc}I & O \\ O & O\end{array}\right]$. For simplicity, we will write $\star$ for the operation $\star_{J}$ on $\mathcal{M}_{m n}^{J}$, throughout. One easily verifies the rule

$$
\left[\begin{array}{ll}
A & B \\
C & D
\end{array}\right] \star\left[\begin{array}{ll}
E & F \\
G & H
\end{array}\right]=\left[\begin{array}{ll}
A E & A F \\
C E & C F
\end{array}\right] .
$$

Also note that if $X=\left[\begin{array}{cc}A & B \\ C & D\end{array}\right]$, then

$$
X J=\left[\begin{array}{ll}
A & O \\
C & O
\end{array}\right] \in \mathcal{M}_{m}, \quad J X=\left[\begin{array}{ll}
A & B \\
O & O
\end{array}\right] \in \mathcal{M}_{n}, \quad J X J=\left[\begin{array}{ll}
A & O \\
O & O
\end{array}\right] \in \mathcal{M}_{n m}
$$

Remark 4.2. In the special case that $r=m \leq n$, we have $J=[I O]$, and the product in $\mathcal{M}_{m n}^{J}$ satisfies $\left[\begin{array}{ll}A & B\end{array}\right] \star\left[\begin{array}{ll}E & F\end{array}\right]=\left[\begin{array}{ll}A E & A F\end{array}\right]$. But we just view this as a special case of the above rule, with the bottom rows - i.e., $\left[\begin{array}{ll}C & D\end{array}\right],\left[\begin{array}{ll}G & H\end{array}\right],[C E C F]$ - containing empty blocks. A dual statement holds in the case $r=n \leq m$. In only one place will we need to consider the case in which $r=\min (m, n)$ separately (see Theorems 4.12 and 4.14). If $r=m=n$, then $\mathcal{M}_{m n}^{J}$ is precisely the full linear monoid $\mathcal{M}_{n}$; since all the problems we investigate have already been solved for $\mathcal{M}_{n}$, we will typically assume that $r=m=n$ does not hold, though our results are true for the case $r<m=n$ (corresponding to variants of the full linear monoids $\mathcal{M}_{n}$ ). See Remark 5.3, where the above observations are used to show that the sandwich semigroups $\mathcal{M}_{m n}^{J}$ are isomorphic to certain well-known (non-sandwich) matrix semigroups in the case that $r=\min (m, n)$.

Green's relations and the regular elements of the sandwich semigroup $\mathcal{M}_{m n}^{J}$ were calculated in [9, 49]. We now show how these results may be recovered (and given a cleaner presentation) using the general theory developed in Section 2. In particular, a crucial role is played by the sets

$P_{1}^{J}=\left\{X \in \mathcal{M}_{m n}: X J \mathscr{R} X\right\}, P_{2}^{J}=\left\{X \in \mathcal{M}_{m n}: J X \mathscr{L} X\right\}, P_{3}^{J}=\left\{X \in \mathcal{M}_{m n}: J X J \mathscr{J} X\right\}, P^{J}=P_{1}^{J} \cap P_{2}^{J}$.

For simplicity, we denote these sets simply by $P_{1}, P_{2}, P_{3}$, and $P=P_{1} \cap P_{2}$.

Certain special matrices from $\mathcal{M}_{m n}$ will be very important in what follows. With this in mind, if $A \in \mathcal{M}_{r}$, $M \in \mathcal{M}_{m-r, r}$ and $N \in \mathcal{M}_{r, n-r}$, we write

$$
[M, A, N]=\left[\begin{array}{cc}
A & A N \\
M A & M A N
\end{array}\right] \in \mathcal{M}_{m n}
$$

One may check that when matrices of this form are multiplied in $\mathcal{M}_{m n}^{J}$, they obey the rule

$$
[M, A, N] \star[K, B, L]=[M, A B, L] .
$$

\section{Proposition 4.3.}

(i) $P_{1}=\left\{X \in \mathcal{M}_{m n}: \operatorname{rank}(X J)=\operatorname{rank}(X)\right\}=\left\{X \in \mathcal{M}_{m n}: \operatorname{Col}(X J)=\operatorname{Col}(X)\right\}$,

(ii) $P_{2}=\left\{X \in \mathcal{M}_{m n}: \operatorname{rank}(J X)=\operatorname{rank}(X)\right\}=\left\{X \in \mathcal{M}_{m n}: \operatorname{Row}(J X)=\operatorname{Row}(X)\right\}$,

(iii) $P_{3}=P=\left\{X \in \mathcal{M}_{m n}: \operatorname{rank}(J X J)=\operatorname{rank}(X)\right\}$

$$
=\left\{[M, A, N]: A \in \mathcal{M}_{r}, M \in \mathcal{M}_{m-r, r}, N \in \mathcal{M}_{r, n-r}\right\},
$$

(iv) $P=\operatorname{Reg}\left(\mathcal{M}_{m n}^{J}\right)$ is the set of all regular elements of $\mathcal{M}_{m n}^{J}$, and is a subsemigroup of $\mathcal{M}_{m n}^{J}$.

Proof. Parts (i) and (ii) follow quickly from Lemma 3.1 (making crucial use of stability). We now prove (iii). Since $\mathcal{M}$ is stable, Proposition 2.11 and Lemma 3.3 give $P_{3}=P=\left\{X \in \mathcal{M}_{m n}: \operatorname{rank}(J X J)=\operatorname{rank}(X)\right\}$. Now let $X=\left[\begin{array}{ll}A & B \\ C & D\end{array}\right] \in \mathcal{M}_{m n}$. First, note that

$$
\begin{aligned}
& X \in P_{2} \Leftrightarrow \operatorname{Row}(X)=\operatorname{Row}(J X)=\operatorname{Row}\left[\begin{array}{cc}
A & B \\
O & O
\end{array}\right] \\
& \Leftrightarrow \text { each row of }\left[\begin{array}{ll}
C & D
\end{array}\right] \text { is a linear combination of the rows of }[A B] \\
& \Leftrightarrow[C D]=M[A B]=[M A M B] \text { for some } M \in \mathcal{M}_{m-r, r} \text {. }
\end{aligned}
$$


Similarly,

$$
X \in P_{1} \Leftrightarrow\left[\begin{array}{l}
B \\
D
\end{array}\right]=\left[\begin{array}{l}
A \\
C
\end{array}\right] N=\left[\begin{array}{l}
A N \\
C N
\end{array}\right] \text { for some } N \in \mathcal{M}_{r, n-r}
$$

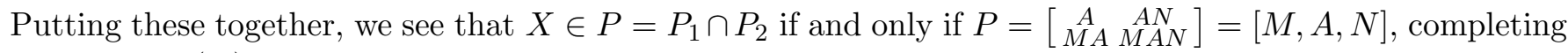
the proof of (iii).

For (iv), Proposition 2.11 gives $\operatorname{Reg}\left(\mathcal{M}_{m n}^{J}\right) \subseteq P$. Conversely, suppose $X=[M, A, N] \in P$. If $B \in \mathcal{M}_{r}$ is such that $A=A B A$ (see Lemma 3.2 , then it is easy to check that $X=X \star Y \star X$ where $Y=\left[\begin{array}{l}B \\ D\end{array}\right]$ for any (appropriately sized) $C, D, E$, completing the proof that $P=\operatorname{Reg}\left(\mathcal{M}_{m n}^{J}\right)$. The fact that $P$ is a subsemigroup follows immediately from Proposition 2.10 and Lemma 3.2 (or directly from the rule $[M, A, N] \star[K, B, L]=$ $[M, A B, L])$.

Remark 4.4. Part (iv) of the previous proposition also follows from [9, Theorem 2.1], but the rest of Proposition 4.3 appears to be new.

Now that we have described the sets $P_{1}, P_{2}, P_{3}=P=P_{1} \cap P_{2}$, we may characterise Green's relations on $\mathcal{M}_{m n}^{J}$. As in Section 2, if $\mathscr{K}$ is one of $\mathscr{R}, \mathscr{L}, \mathscr{H}, \mathscr{D}, \mathscr{J}$, we will write $\mathscr{K}^{J}$ for the Green's $\mathscr{K}$-relation on $\mathcal{M}_{m n}^{J}$. Since $\mathcal{M}_{m n}^{J}$ is not a monoid in general, these relations are defined, for $X, Y \in \mathcal{M}_{m n}$, by

- $X \mathscr{R}^{J} Y \Leftrightarrow[X=Y]$ or $\left[X=Y \star U\right.$ and $Y=X \star V$ for some $\left.U, V \in \mathcal{M}_{m n}\right]$,

and so on. Since $\mathcal{M}$ is stable, so too is $\mathcal{M}_{m n}^{J}$, so we have $\mathscr{J}^{J}=\mathscr{D}^{J}$ (see Proposition 2.14 and Lemmas 2.6 and 3.1). We will continue to write $\mathscr{R}, \mathscr{L}, \mathscr{H}, \mathscr{D}, \mathscr{J}$ for the relations on $\mathcal{M}$ defined in Section 3 , As in Section 2 , if $\mathscr{K}$ is one of $\mathscr{R}, \mathscr{L}, \mathscr{H}, \mathscr{D}=\mathscr{J}$, and if $X \in \mathcal{M}_{m n}$, we will write

$$
K_{X}=\left\{Y \in \mathcal{M}_{m n}: X \mathscr{K} Y\right\} \quad \text { and } \quad K_{X}^{J}=\left\{Y \in \mathcal{M}_{m n}: X \mathscr{K}^{J} Y\right\}
$$

for the $\mathscr{K}$-class and $\mathscr{K}^{J}$-class of $X$ in $\mathcal{M}_{m n}$, respectively. As noted in Section 2 , $\mathscr{K}^{J} \subseteq \mathscr{K}$ for each $\mathscr{K}$, and so $K_{X}^{J} \subseteq K_{X}$ for each $X$. The next result follows immediately from Theorem 2.13 . It also follows from Theorem 2.3, Lemma 2.4, and Corollaries 2.5-2.8 of [9], but we prefer the current succinct description.

Theorem 4.5. If $X \in \mathcal{M}_{m n}$, then

(i) $R_{X}^{J}= \begin{cases}R_{X} \cap P_{1} & \text { if } X \in P_{1} \\ \{X\} & \text { if } X \in \mathcal{M}_{m n} \backslash P_{1}\end{cases}$

(iii) $H_{X}^{J}= \begin{cases}H_{X} & \text { if } X \in P \\ \{X\} & \text { if } X \in \mathcal{M}_{m n} \backslash P\end{cases}$

(ii) $L_{X}^{J}= \begin{cases}L_{X} \cap P_{2} & \text { if } X \in P_{2} \\ \{X\} & \text { if } X \in \mathcal{M}_{m n} \backslash P_{2},\end{cases}$

(iv) $D_{X}^{J}= \begin{cases}D_{X} \cap P & \text { if } X \in P \\ L_{X}^{J} & \text { if } X \in P_{2} \backslash P_{1} \\ R_{X}^{J} & \text { if } X \in P_{1} \backslash P_{2} \\ \{X\} & \text { if } X \in \mathcal{M}_{m n} \backslash\left(P_{1} \cup P_{2}\right) .\end{cases}$

The sets $P_{1}, P_{2}$ are described in Proposition 4.3, and the sets $R_{X}, L_{X}, H_{X}, D_{X}$ in Proposition 3.3 . In particular, $R_{X}^{J}=L_{X}^{J}=H_{X}^{J}=D_{X}^{J}=\{X\}$ if $\operatorname{rank}(X)>r$. If $X \in \mathcal{M}_{m n} \backslash P$, then $H_{X}^{J}=\{X\}$ is a non-group $\mathscr{H}^{J}$-class of $\mathcal{M}_{m n}^{J}$.

Eggbox diagrams of some linear sandwich semigroups are given in Figures 4 and 5 . As usual, grey boxes indicate group $\mathscr{H}^{J}$-classes; a label of $\mathrm{k}$ on such a group $\mathscr{H}^{J}$-class indicates isomorphism to $\mathcal{G}_{k}$. Note that the bottom diagram from Figure 4 is of a variant of $\mathcal{M}_{3}\left(\mathbb{Z}_{2}\right)=\mathcal{M}_{33}\left(\mathbb{Z}_{2}\right)$. The diagrams in the pdf version of this article may be zoomed in a long way. The authors may be contacted for more such pictures.

Theorem 4.5 yields an intuitive picture of the internal structure of $\mathcal{M}_{m n}^{J}$. Recall that the $\mathscr{D}$-classes of $\mathcal{M}_{m n}$ are the sets $D_{s}\left(\mathcal{M}_{m n}\right)=\left\{X \in \mathcal{M}_{m n}: \operatorname{rank}(X)=s\right\}$ for $0 \leq s \leq l=\min (m, n)$. If $r<l$, then each of the $\mathscr{D}$-classes $D_{r+1}\left(\mathcal{M}_{m n}\right), \ldots, D_{l}\left(\mathcal{M}_{m n}\right)$ separates completely into singleton $\mathscr{D}^{J}$-classes in $\mathcal{M}_{m n}^{J}$. (We will study these classes in more detail shortly.) Next, note that $D_{0}\left(\mathcal{M}_{m n}\right)=\{O\} \subseteq P$ (as the zero matrix clearly belongs to both $P_{1}$ and $\left.P_{2}\right)$, so $D_{0}\left(\mathcal{M}_{m n}\right)$ remains a (regular) $\mathscr{D}^{J}$-class of $\mathcal{M}_{m n}^{J}$. Now fix some 


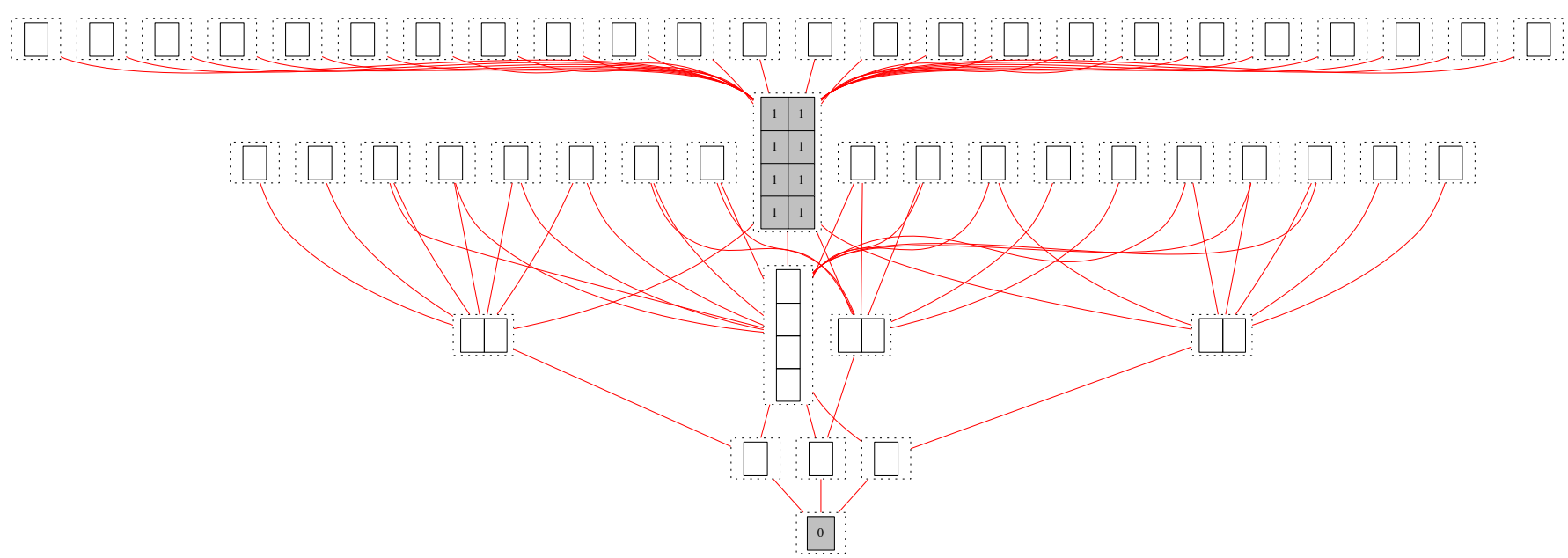

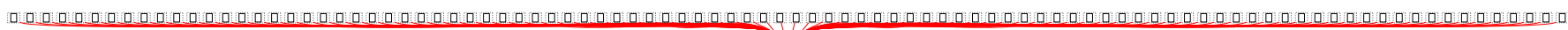
:

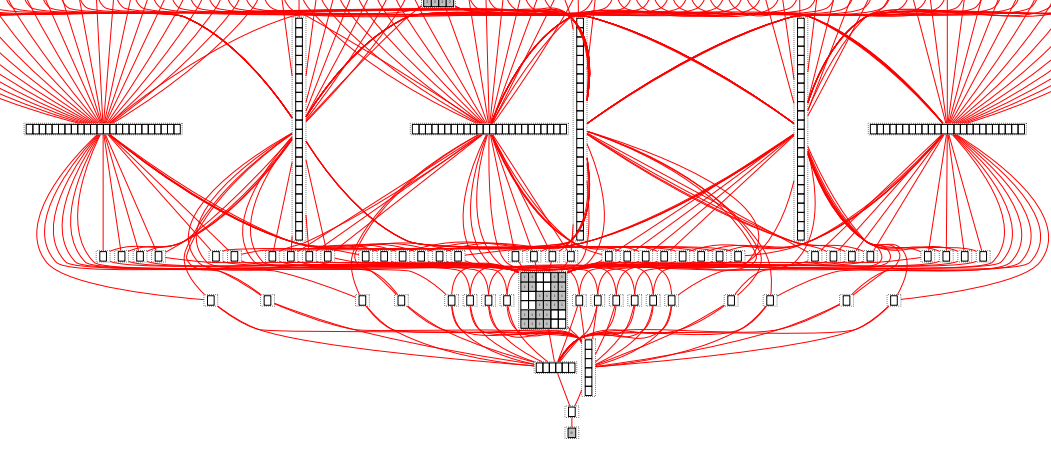

Figure 4: Egg box diagrams of the linear sandwich semigroups $\mathcal{M}_{32}^{J_{231}}\left(\mathbb{Z}_{2}\right)$ and $\mathcal{M}_{33}^{J_{332}}\left(\mathbb{Z}_{2}\right)$ (top and bottom, respectively).

$1 \leq s \leq r$. The $\mathscr{D}$-class $D_{s}\left(\mathcal{M}_{m n}\right)$ is split into a single regular $\mathscr{D}^{J}$-class, namely $D_{s}\left(\mathcal{M}_{m n}\right) \cap P$, and a number of non-regular $\mathscr{D}^{J}$-classes. Some of these non-regular $\mathscr{D}^{J}$-classes are singletons, namely those of the form $D_{X}^{J}=\{X\}$ where $X \in D_{s}\left(\mathcal{M}_{m n}\right)$ belongs to neither $P_{1}$ nor $P_{2}$. Some of the non-regular $\mathscr{D}^{J}$-classes consist of one non-singleton $\mathscr{L}^{J}$-class, namely those of the form $D_{X}^{J}=L_{X}^{J}=L_{X} \cap P_{2}$, where $X \in D_{s}\left(\mathcal{M}_{m n}\right)$ belongs to $P_{2} \backslash P_{1}$; the $\mathscr{H}^{J}$-classes contained in such a $\mathscr{D}^{J}$-class are all singletons. The remaining nonregular $\mathscr{D}^{J}$-classes contained in $D_{s}\left(\mathcal{M}_{m n}\right)$ consist of one non-singleton $\mathscr{R}^{J}$-class, namely those of the form $D_{X}^{J}=R_{X}^{J}=R_{X} \cap P_{1}$, where $X \in D_{s}\left(\mathcal{M}_{m n}\right)$ belongs to $P_{1} \backslash P_{2}$; the $\mathscr{H}^{J}$-classes contained in such a $\mathscr{D}^{J}$-class are all singletons. This is all pictured in Figure 6 for the $\mathscr{D}$-class $D_{1}\left(\mathcal{M}_{23}\right)$ where $\mathbb{F}=\mathbb{Z}_{3}=\{0,1,2\}$ and $J=J_{321}=\left[\begin{array}{ll}1 & 0 \\ 0 & 0 \\ 0 & 0\end{array}\right] ;$ cf. Figure 2 .

It will be important to have a description of the partial order $\leq$ on the $\mathscr{D}^{J}$-classes of $\mathcal{M}_{m n}^{J}$.

Proposition 4.6. Let $X, Y \in \mathcal{M}_{m n}$. Then $D_{X}^{J} \leq D_{Y}^{J}$ in $\mathcal{M}_{m n}^{J}$ if and only if one of the following holds:

(i) $X=Y$,

(ii) $\operatorname{rank}(X) \leq \operatorname{rank}(J Y J)$, (iii) $\operatorname{Row}(X) \subseteq \operatorname{Row}(J Y)$,

(iv) $\operatorname{Col}(X) \subseteq \operatorname{Col}(Y J)$.

Proof. Note that $D_{X}^{J} \leq D_{Y}^{J}$ if and only if one of the following holds:
(a) $X=Y$,
(c) $X=U J Y$ for some $U \in \mathcal{M}_{m n}$,
(b) $X=U J Y J V$ for some $U, V \in \mathcal{M}_{m n}$,
(d) $X=Y J V$ for some $V \in \mathcal{M}_{m n}$.

The equivalences (b) $\Leftrightarrow$ (ii), (c) $\Leftrightarrow$ (iii), and (d) $\Leftrightarrow$ (iv) all follow from Lemma 3.1 .

The description of the order on $\mathscr{D}^{J}$-classes of $\mathcal{M}_{m n}^{J}$ from Proposition 4.6 may be simplified in the case that one of $X, Y$ is regular. 

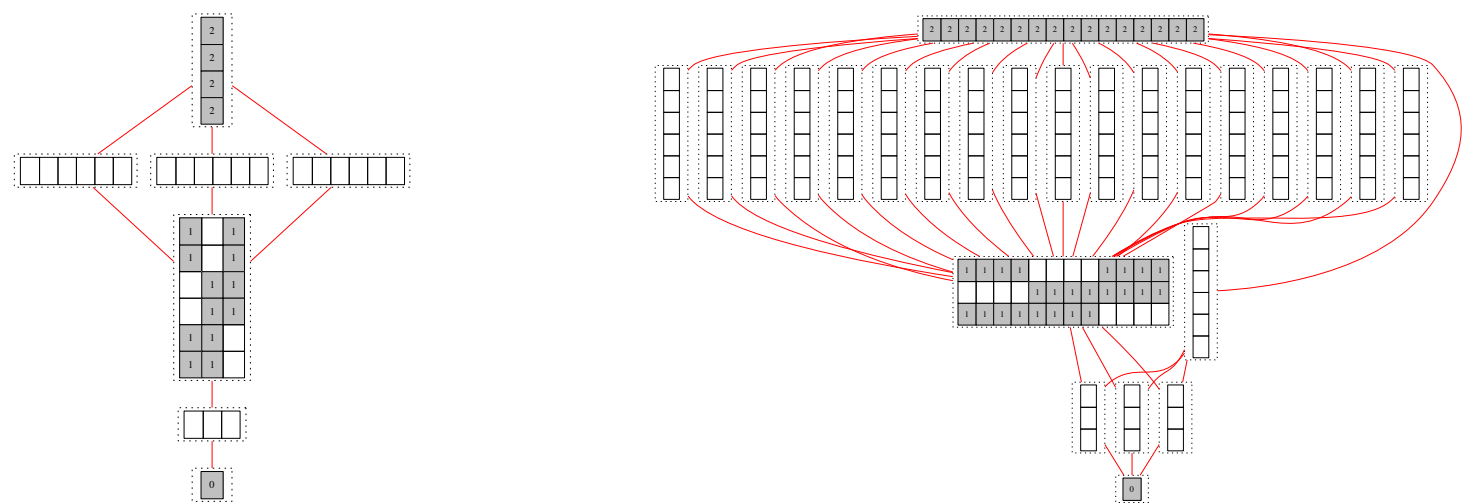

Figure 5: Egg box diagrams of the linear sandwich semigroups $\mathcal{M}_{32}^{J_{232}}\left(\mathbb{Z}_{2}\right)$ and $\mathcal{M}_{24}^{J_{422}}\left(\mathbb{Z}_{2}\right.$ ) (left and right, respectively).

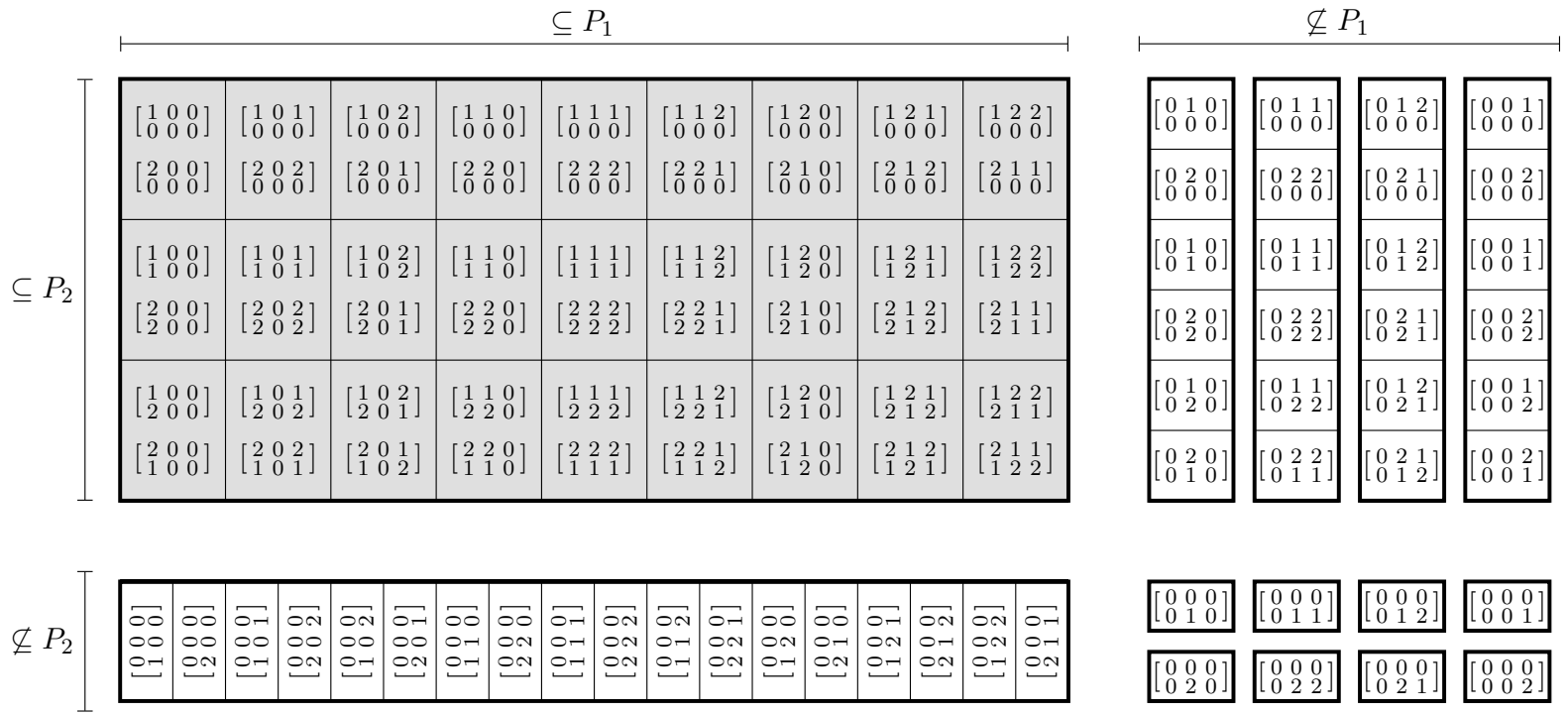

Figure 6: A $\mathscr{D}$-class $D_{1}\left(\mathcal{M}_{23}\left(\mathbb{Z}_{3}\right)\right)$ breaks up into $\mathscr{D}^{J}$-classes in $\mathcal{M}_{23}^{J}\left(\mathbb{Z}_{3}\right)$, where $J=J_{321}$. Group $\mathscr{H}^{J}$-classes are shaded grey; the idempotent of such a group is the upper of the two matrices. (cf. Figure 2 .)

Proposition 4.7. Let $X, Y \in \mathcal{M}_{m n}$.

(i) If $X \in P$, then $D_{X}^{J} \leq D_{Y}^{J} \Leftrightarrow \operatorname{rank}(X) \leq \operatorname{rank}(J Y J)$.

(ii) If $Y \in P$, then $D_{X}^{J} \leq D_{Y}^{J} \Leftrightarrow \operatorname{rank}(X) \leq \operatorname{rank}(Y)$.

The regular $\mathscr{D}^{J}$-classes of $\mathcal{M}_{m n}^{J}$ form a chain: $D_{0}^{J}<\cdots<D_{r}^{J}$, where

$$
D_{s}^{J}=D_{s}\left(\mathcal{M}_{m n}\right) \cap P=\{X \in P: \operatorname{rank}(X)=s\} \quad \text { for each } 0 \leq s \leq r .
$$

Proof. As in the proof of Proposition 4.6, $D_{X}^{J} \leq D_{Y}^{J}$ if and only if one of (a-d) holds. Suppose first that $X \in P$, so $X=X J Z J X$ for some $Z \in \mathcal{M}_{m n}$. Then (a) implies $X=X J Z(J Y J) Z J X$, (c) implies $X=U(J Y J) Z J X$, and (d) implies $X=X J Z(J Y J) V$. So, in each of cases (a-d), we deduce that $\operatorname{rank}(X) \leq \operatorname{rank}(J Y J)$. So $D_{X}^{J} \leq D_{Y}^{J}$ implies $\operatorname{rank}(X) \leq \operatorname{rank}(Y J Y)$. Proposition 4.6 gives the reverse implication.

Next, suppose $Y \in P$. Now, each of (a-d) implies $\operatorname{rank}(X) \leq \operatorname{rank}(Y)$. Conversely, if $\operatorname{rank}(X) \leq \operatorname{rank}(Y)$, then Proposition 4.6 gives $D_{X}^{J} \leq D_{Y}^{J}$, since $\operatorname{rank}(Y)=\operatorname{rank}(J Y J)$. The statement about regular $\mathscr{D}^{J}$-classes follows quickly from (ii).

The linear ordering on the regular $\mathscr{D}^{J}$-classes may be seen by inspecting Figures 4 and 5 ; see also Figure 8 . 
As an immediate consequence of Proposition 4.7. we may classify the isomorphism classes of sandwich semigroups on the set $\mathcal{M}_{m n}$; the $m=n$ case of the next result was proved in [48].

Corollary 4.8. Let $A, B \in \mathcal{M}_{n m}$. Then $\mathcal{M}_{m n}^{A} \cong \mathcal{M}_{m n}^{B}$ if and only if $\operatorname{rank}(A)=\operatorname{rank}(B)$.

Proof. Put $r=\operatorname{rank}(A)$ and $s=\operatorname{rank}(B)$. By Proposition 4.7 and Lemma 4.1(ii), $\mathcal{M}_{m n}^{A} \cong \mathcal{M}_{m n}^{J_{n m r}}$ and $\mathcal{M}_{m n}^{B} \cong \mathcal{M}_{m n}^{J_{n m s}}$ have $r+1$ and $s+1$ regular $\mathscr{D}^{A}$ - and $\mathscr{D}^{B}$-classes, respectively. So $\mathcal{M}_{m n}^{A} \cong \mathcal{M}_{m n}^{B}$ implies $r=s$. The converse was proved in Lemma 4.1(ii).

Remark 4.9. It is possible to have $\mathcal{M}_{m n}^{A} \cong \mathcal{M}_{k l}^{B}$ even if $(m, n) \neq(k, l)$, although we would of course still need $\operatorname{rank}(A)=\operatorname{rank}(B)$ by Proposition 4.7. For example, if $O=O_{n m}$ is the $n \times m$ zero matrix, then $\mathcal{M}_{m n}^{O}$ is a zero semigroup $\left(X \star Y=O_{m n}\right.$ for all $\left.X, Y \in \mathcal{M}_{m n}\right)$. Two such zero semigroups $\mathcal{M}_{m n}^{O}$ and $\mathcal{M}_{k l}^{O}$ are isomorphic if and only if they have the same cardinality; that is, if and only if $\mathbb{F}$ is infinite or $\mathbb{F}$ is finite and $m n=k l$. We will return to the problem of distinguishing non-isomorphic $\mathcal{M}_{m n}^{A}$ and $\mathcal{M}_{k l}^{B}$ in Theorem 6.5. See Figure 7.

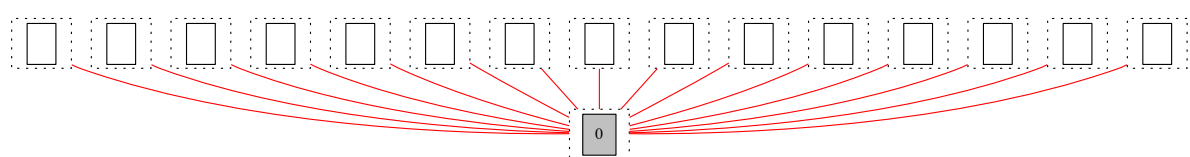

Figure 7: Egg box diagram of the linear sandwich semigroup $\mathcal{M}_{22}^{O_{22}}\left(\mathbb{Z}_{2}\right)$ or, equivalently, $\mathcal{M}_{21}^{O_{12}}\left(\mathbb{F}_{4}\right)$. Both are zero semigroups of size 16.

The next result describes the maximal $\mathscr{D}^{J}$-classes of $\mathcal{M}_{m n}^{J}$. See also Figures 4 and 5

Proposition 4.10. (i) If $r=\min (m, n)$, then $D_{r}^{J}=D_{r} \cap P=\{X \in P: \operatorname{rank}(X)=r\}$ is the unique maximal $\mathscr{D}^{J}$-class of $\mathcal{M}_{m n}^{J}$, and is a subsemigroup of $\mathcal{M}_{m n}^{J}$.

(ii) If $r<\min (m, n)$, then the maximal $\mathscr{D}^{J}$-classes of $\mathcal{M}_{m n}^{J}$ are those of the form $D_{X}^{J}=\{X\}$ with $\operatorname{rank}(X)>r$.

Proof. Part (i) follows immediately from Proposition 4.7(ii), the rule $[M, A, N] \star[K, B, L]=[M, A B, L]$, and the fact that $\mathcal{G}_{r}=D_{r}\left(\mathcal{M}_{r}\right)$ is a subgroup of $\mathcal{M}_{r}$.

For (ii), let $X \in \mathcal{M}_{m n}$. Suppose first that $\operatorname{rank}(X)>r$ and that $D_{X}^{J} \leq D_{Y}^{J}$. Then condition (ii) from Proposition 4.6 does not hold, $\operatorname{since} \operatorname{rank}(J Y J) \leq \operatorname{rank}(J)=r<\operatorname{rank}(X) . \operatorname{Similarly}, \operatorname{rank}(J Y)<\operatorname{rank}(X)$ and $\operatorname{rank}(Y J)<\operatorname{rank}(X)$, so neither (iii) nor (iv) holds. Having eliminated (ii-iv), we deduce that (i) must hold; that is, $X=Y$, so $D_{X}^{J}=\{X\}$ is indeed maximal. Conversely, suppose $\operatorname{rank}(X) \leq r$, and let $Y=\left[\begin{array}{cc}I_{r} & O \\ O & D\end{array}\right]$, where $D \neq O$. Then $\operatorname{rank}(Y)>r$, so $D_{Y}^{J}=\{Y\}$ is maximal by the previous paragraph. But also $J Y J=J$, and it follows that $\operatorname{rank}(X) \leq r=\operatorname{rank}(J)=\operatorname{rank}(J Y J)$, so that $D_{X}^{J}<D_{Y}^{J}=\{Y\}$, whence $D_{X}^{J}$ is not maximal.

The description of the maximal $\mathscr{D}^{J}$-classes from Proposition 4.10 allows us to obtain information about generating sets for $\mathcal{M}_{m n}^{J}$ and, in the case of finite $\mathbb{F}$, about $\operatorname{rank}\left(\mathcal{M}_{m n}^{J}\right)$. In order to avoid confusion when discussing generation, if $\Omega \subseteq \mathcal{M}_{m n}$, we will write $\langle\Omega\rangle_{J}$ for the subsemigroup of $\mathcal{M}_{m n}^{J}$ generated by $\Omega$, which consists of all products $X_{1} \star \cdots \star X_{k}$, with $k \geq 1$ and $X_{1}, \ldots, X_{k} \in \Omega$. If $\Sigma \subseteq \mathcal{M}_{k}$ for some $k$, we will continue to write $\langle\Sigma\rangle$ for the subsemigroup of $\mathcal{M}_{k}$ generated by $\Sigma$. For convenience, we will state two separate results, according to whether $r=\min (m, n)$ or $r<\min (m, n)$. The next lemma will be useful as the inductive step in the proofs of both Theorems 4.12 and 4.14 . Recall that $\left\{e_{m 1}, \ldots, e_{m m}\right\}$ is the standard basis of $V_{m}=\mathbb{F}^{m}$.

Lemma 4.11. Suppose $X \in D_{s}\left(\mathcal{M}_{m n}\right)$, where $0 \leq s \leq l-1$ and $l=\min (m, n)$. Then $X=Y \star Z$ for some $Y \in D_{l}\left(\mathcal{M}_{m n}\right)$ and $Z \in D_{s+1}\left(\mathcal{M}_{m n}\right)$. 
Proof. Let $\mathscr{B}=\left\{v_{1}, \ldots, v_{n}\right\}$ be a basis of $V_{n}$ such that $\left\{v_{s+1}, \ldots, v_{n}\right\}$ is a basis of $\operatorname{ker}\left(\lambda_{X}\right)$. Consider the linear transformation $\beta \in \operatorname{Hom}\left(V_{n}, V_{m}\right)$ defined by

$$
\beta\left(v_{i}\right)= \begin{cases}e_{m i} & \text { if } 1 \leq i \leq s \\ 0 & \text { if } s<i<n \\ e_{m m} & \text { if } i=n\end{cases}
$$

noting that $\operatorname{rank}(\beta)=s+1$. The proof now breaks into two cases, depending on whether $r<m$ or $r=m$.

Case 1. Suppose first that $r<m$. Let $\alpha \in \operatorname{Hom}\left(V_{n}, V_{m}\right)$ be any linear transformation of rank $l$ that extends the map $e_{n i} \mapsto \lambda_{X}\left(v_{i}\right)(1 \leq i \leq s)$. One easily checks that $\alpha \circ \lambda_{J} \circ \beta=\lambda_{X}$.

Case 2. Now suppose $r=m$. Recall that we are assuming that $r=m=n$ does not hold, so $r=m<n$. This time, define we let $\alpha$ be any linear transformation of rank $m=l$ that extends the map $e_{n i} \mapsto \lambda_{X}\left(v_{i}\right)$ $(1 \leq i \leq s), e_{n r}=e_{n m} \mapsto 0$. Then, again, one easily checks that $\alpha \circ \lambda_{J} \circ \beta=\lambda_{X}$.

Theorem 4.12. Suppose $r<l=\min (m, n)$. Then $\mathcal{M}_{m n}^{J}=\langle\Omega\rangle_{J}$, where $\Omega=\left\{X \in \mathcal{M}_{m n}: \operatorname{rank}(X)>r\right\}$. Further, any generating set for $\mathcal{M}_{m n}^{J}$ contains $\Omega$. If $|\mathbb{F}|=q<\infty$, then

$$
\operatorname{rank}\left(\mathcal{M}_{m n}^{J}\right)=|\Omega|=\sum_{s=r+1}^{l}\left[\begin{array}{c}
m \\
s
\end{array}\right]_{q}\left[\begin{array}{c}
n \\
s
\end{array}\right]_{q} q^{\left(\begin{array}{c}
s \\
2
\end{array}\right)}(q-1)^{s}[s]_{q} !
$$

Proof. For convenience, we will assume that $l=m \leq n$. The other case will follow by duality. We will also denote $D_{s}\left(\mathcal{M}_{m n}\right)$ simply by $D_{s}$ for each $0 \leq s \leq m$. Consider the statement:

$$
H(s): \quad\langle\Omega\rangle_{J} \text { contains } D_{s} \cup \cdots \cup D_{m}=\left\{X \in \mathcal{M}_{m n}: \operatorname{rank}(X) \geq s\right\} .
$$

Note that $\Omega=D_{r+1} \cup \cdots \cup D_{m}$, so $H(s)$ is clearly true for $r+1 \leq s \leq m$. Lemma 4.11 shows that $H(s+1)$ implies $H(s)$ for all $0 \leq s \leq m-1$. So we conclude that $H(s)$ is true for all $0 \leq s \leq m$. In particular, $H(0)$ says that $\mathcal{M}_{m n}^{J}=\langle\Omega\rangle_{J}$.

Since $\{X\}$ is a maximal $\mathscr{D}^{J}$-class for any $X \in \Omega$, it follows that any generating set of $\mathcal{M}_{m n}^{J}$ must contain $\Omega$. Thus, $\Omega$ is the minimal generating set with respect to both size and containment, $\operatorname{so} \operatorname{rank}\left(\mathcal{M}_{m n}^{J}\right)=|\Omega|$. The formula for $|\Omega|$ with $|\mathbb{F}|$ finite follows from Lemma 3.4 .

In order to consider the case in which $r=\min (m, n)$, we first prove an intermediate result. There is a dual version of the following lemma (dealing with the case in which $r=n<m$ ), but we will not state it.

Lemma 4.13. If $r=m<n$, then
(i) $P_{2}=\mathcal{M}_{m n}^{J}$,
(ii) $P=P_{1}$ is a left ideal of $\mathcal{M}_{m n}^{J}$,
(iii) $\mathscr{L}^{J}=\mathscr{L}$ in $\mathcal{M}_{m n}^{J}$.

Proof. Let $X \in \mathcal{M}_{m n}^{J}$. As noted earlier, in the $2 \times 2$ block description, $X=\left[\begin{array}{cc}A & B \\ C & D\end{array}\right]$ (where $A \in \mathcal{M}_{r}$, and so on), the matrices $C$ and $D$ are empty $($ since $r=m)$. So we write $X=[A B]$. Note that $J=\left[\begin{array}{l}I \\ O\end{array}\right]$, so $J X=\left[\begin{array}{l}I \\ O\end{array}\right]\left[\begin{array}{ll}A & B\end{array}\right]=\left[\begin{array}{cc}A & B \\ O & O\end{array}\right]$. It follows that $\operatorname{Row}(J X)=\operatorname{Row}(X)$ and, since $X \in \mathcal{M}_{m n}^{J}$ was arbitrary, this completes the proof of (i).

We immediately deduce $P=P_{1}$ from (i). As in Proposition 4.3, the regular elements of $\mathcal{M}_{m n}^{J}$ are of the form $[A A N]$ where $A \in \mathcal{M}_{r}$ and $N \in \mathcal{M}_{r, n-r}$. We denote such a regular element by $[A, N]$. The proof of (ii) concludes with the easily checked observation that $[A B] \star[C, N]=[A C, N]$.

Part (iii) follows quickly from (i) and Theorem 4.5(ii).

Theorem 4.14. Suppose $r=\min (m, n)$ where $m \neq n$. If $|\mathbb{F}|=q<\infty$, then

$$
\operatorname{rank}\left(\mathcal{M}_{m n}^{J}\right)=\left[\begin{array}{c}
L \\
l
\end{array}\right]_{q},
$$

where $L=\max (m, n)$ and $l=\min (m, n)$. 
Proof. Again, it suffices to assume that $r=m<n$, so $l=m$ and $L=n$. We keep the notation of the previous proof.

Let $\Omega$ be an arbitrary generating set for $\mathcal{M}_{m n}^{J}$. Let $X \in D_{m}\left(\mathcal{M}_{m n}\right)$ be arbitrary. We claim that $\Omega$ must contain some element of $L_{X}^{J}=L_{X}$. Indeed, consider an expression $X=Y_{1} \star \cdots \star Y_{k}$, where $Y_{1}, \ldots, Y_{k} \in \Omega$. If $k=1$, then $Y_{1}=X \in L_{X}$ and the claim is established, so suppose $k \geq 2$. Since $D_{m}\left(\mathcal{M}_{m n}\right)$ is a maximal $\mathscr{D}^{J}$ class, we must have $Y_{k} \in D_{m}\left(\mathcal{M}_{m n}\right)$. So $Y_{k} \mathscr{D}^{J} X=\left(Y_{1} \star \cdots \star Y_{k-1}\right) \star Y_{k}$, whence $Y_{k} \mathscr{L}^{J}\left(Y_{1} \star \cdots \star Y_{k-1}\right) \star Y_{k}=X$, by stability. By Lemma 4.13 (iii), this completes the proof of the claim. In particular, $|\Omega|$ is bounded below by the number of $\mathscr{L}$-classes contained in $D_{m}\left(\mathcal{M}_{m n}\right)$, which is equal to $\left[{ }_{m}^{n}\right]_{q}$, by Lemma 3.4 . Since $\Omega$ was an arbitrary generating set, it follows that $\operatorname{rank}\left(\mathcal{M}_{m n}^{J}\right) \geq\left[\begin{array}{l}n \\ m\end{array}\right]_{q}=\left[\begin{array}{l}L \\ l\end{array}\right]_{q}$.

To complete the proof, it remains to check that there exists a generating set of the desired cardinality. For each $N \in \mathcal{M}_{m, n-m}$, choose some $A_{N} \in \mathcal{G}_{r}$ such that $\left\{A_{N}: N \in \mathcal{M}_{m, n-m}\right\}$ generates $\mathcal{G}_{m}$, and put $X_{N}=\left[A_{N}, N\right] \in D_{m}^{J}$. (This is possible since $\left|\mathcal{M}_{m, n-m}\right|=q^{m(n-m)} \geq 2$, and $\operatorname{rank}\left(\mathcal{G}_{m}\right) \leq 2$ by Theorem 3.7 .) It is easy to see that $\Omega_{1}=\left\{X_{N}: N \in \mathcal{M}_{m, n-m}\right\}$ is a cross-section of the $\mathscr{L}$-classes in $D_{m}^{J}$. Also, choose some cross-section $\Omega_{2}=\left\{Y_{i}: i \in I\right\}$ of the $\mathscr{L}$-classes contained in $D_{m}\left(\mathcal{M}_{m n}\right) \backslash D_{m}^{J}$. Then $\Omega=\Omega_{1} \cup \Omega_{2}$ is a cross-section of the $\mathscr{L}$-classes contained in $D_{m}\left(\mathcal{M}_{m n}\right)$. Since, therefore, $|\Omega|=\left[{ }_{m}^{n}\right]_{q}$, the proof will be complete if we can show that $\mathcal{M}_{m n}^{J}=\langle\Omega\rangle_{J}$. By Lemma 4.11, it suffices to show that $\langle\Omega\rangle_{J}$ contains $D_{m}\left(\mathcal{M}_{m n}\right)$. So suppose $Z \in D_{m}\left(\mathcal{M}_{m n}\right)$. Assume first that $Z \in D_{m}^{J}$, and write $Z=[B, L]$, noting that $B \in \mathcal{G}_{r}$. Choose $N_{1}, \ldots, N_{k} \in \mathcal{M}_{m, n-m}$ such that $B A_{L}^{-1}=A_{N_{1}} \cdots A_{N_{k}}$. Then one easily checks that $Z=X_{N_{1}} \star \cdots \star X_{N_{k}} \star X_{L}$. Now, suppose $Z$ is not regular. Choose $i \in I$ such that $Z \mathscr{L} Y_{i}$. By Lemma 3.3 , $Z=U Y_{i}$ for some $U \in \mathcal{G}_{m}$. But then $Z=[U V] \star Y_{i}$ for any $V \in \mathcal{M}_{m, n-m}$. Since $\operatorname{rank}(U)=m$, we have $[U V] \in D_{m}^{J} \subseteq\langle\Omega\rangle_{J}$, whence $Z \in\langle\Omega\rangle_{J}$, completing the proof.

Remark 4.15. By inspecting Figures 4 and 5 , the reader may use Theorems 4.12 and 4.14 to locate the elements from a minimal generating set for $\mathcal{M}_{m n}^{J}$.

\section{Connection to (non-sandwich) matrix semigroups}

Recall that $J=J_{n m r}=\left[\begin{array}{cc}I_{r} & O_{r, n-r} \\ O_{m-r, r} & O_{m-r, n-r}\end{array}\right] \in \mathcal{M}_{n m}$. Now let $K=J^{T}=J_{m n r}=\left[\begin{array}{cc}I_{r} & O_{r, m-r} \\ O_{n-r, r} & O_{n-r, m-r}\end{array}\right] \in \mathcal{M}_{m n}$. So Lemma 4.1 says that $\mathcal{M}_{n m}^{K}$ and $\mathcal{M}_{m n}^{J}$ are anti-isomorphic. Also, since $J=J K J$ and $K=K J K$, Theorem 2.15 says that we have the following commutative diagrams of semigroup homomorphisms where, for clarity, we write $\cdot$ for (non-sandwich) matrix multiplication:
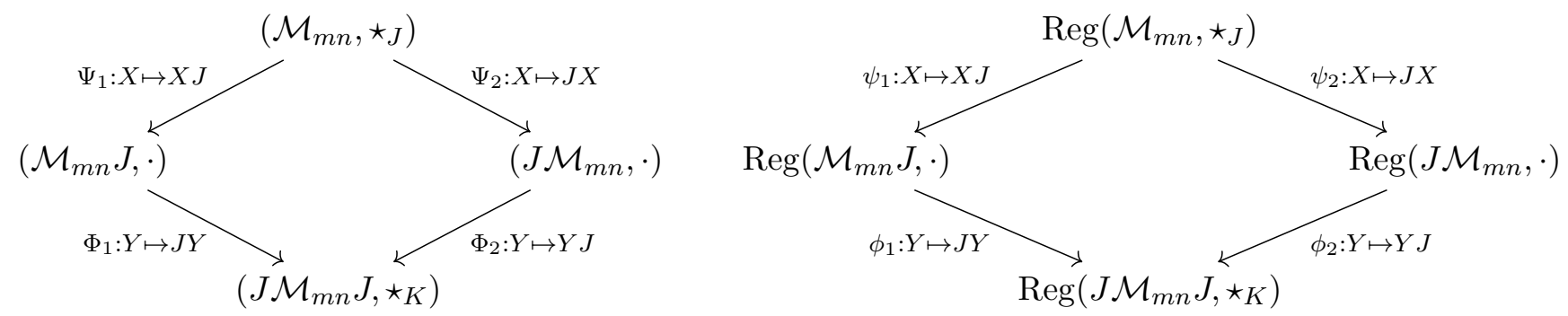

In this section, we show that the various semigroups appearing in the above diagrams are all (equal to or isomorphic to) certain well-known (non-sandwich) matrix semigroups, and explore the consequences for the structure of the sandwich semigroups $\mathcal{M}_{m n}^{J}$. First, we have a simple observation.

Lemma 5.1. We have $\left(J \mathcal{M}_{m n} J, \star_{K}\right)=\operatorname{Reg}\left(J \mathcal{M}_{m n} J, \star_{K}\right) \cong\left(\mathcal{M}_{r}, \cdot\right)$.

Proof. Let $X=\left[\begin{array}{ll}A & B \\ C & D\end{array}\right] \in \mathcal{M}_{m n}$. We have already observed that, whether $X$ is regular or not, $J X J=$ $\left[\begin{array}{ll}A & O \\ O & O\end{array}\right] \in \mathcal{M}_{n m}$. The result follows quickly from the fact that $\left[\begin{array}{cc}A & O \\ O & O\end{array}\right] \star_{K}\left[\begin{array}{cc}E & O \\ O & O\end{array}\right]=\left[\begin{array}{cc}A E & O \\ O & O\end{array}\right]$.

For integers $k \geq 1$ and $0 \leq l \leq k$, we write

$$
\begin{aligned}
\mathcal{C}_{k}(l) & =\left\{X \in \mathcal{M}_{k}: \mathfrak{c}_{l+1}(X)=\cdots=\mathfrak{c}_{k}(X)=O\right\}, \\
\mathcal{R}_{k}(l) & =\left\{X \in \mathcal{M}_{k}: \mathfrak{r}_{l+1}(X)=\cdots=\mathfrak{r}_{k}(X)=O\right\} .
\end{aligned}
$$


(As before, without causing confusion, we write $O$ for any zero matrix when the dimensions are clear from context.) These matrix semigroups have been studied in a number of contexts (see for example [72, 89]), along with their associated isomorphic semigroups of linear transformations

$$
\begin{aligned}
\mathcal{K}_{k}(l) & =\left\{\alpha \in \operatorname{End}\left(V_{k}\right): \operatorname{ker}(\alpha) \supseteq W_{k l}^{\perp}\right\}, \\
\mathcal{I}_{k}(l) & =\left\{\alpha \in \operatorname{End}\left(V_{k}\right): \operatorname{im}(\alpha) \subseteq W_{k l}\right\} .
\end{aligned}
$$

Here we have written $W_{k l}^{\perp}=\operatorname{span}\left\{e_{k, l+1}, \ldots, e_{k k}\right\}$. Clearly, $\mathcal{C}_{k}(l)$ and $\mathcal{R}_{k}(l)$ are anti-isomorphic.

Lemma 5.2. We have $\mathcal{M}_{m n} J=\mathcal{C}_{m}(r)$ and $J \mathcal{M}_{m n}=\mathcal{R}_{n}(r)$.

Proof. Let $X=\left[\begin{array}{cc}A & B \\ C & D\end{array}\right] \in \mathcal{M}_{m n}$. We have already observed that $X J=\left[\begin{array}{ll}A & O \\ C & O\end{array}\right] \in \mathcal{M}_{m}$ and $J X=\left[\begin{array}{ll}A & B \\ O & O\end{array}\right]$, and the result quickly follows.

Remark 5.3. A typical element $X \in \mathcal{R}_{k}(l)$ may be written as $X=\left[\begin{array}{cc}A & B \\ O & O\end{array}\right]$, where $A \in \mathcal{M}_{l}, B \in \mathcal{M}_{l, k-l}$ and so on. One easily checks that multiplication of matrices in this form obeys the rule $\left[\begin{array}{cc}A & B \\ O & O\end{array}\right]\left[\begin{array}{cc}E & F \\ O & O\end{array}\right]=\left[\begin{array}{ccc}A E & A F \\ O & O\end{array}\right]$. Comparing this to the discussion in Remark 4.2, we see that $\mathcal{R}_{k}(l)$ is isomorphic to the sandwich semigroup $\mathcal{M}_{l k}^{J}$ where $J=J_{k l l} \in \mathcal{M}_{k l}$. (A dual statement holds for the matrix semigroups $\mathcal{C}_{k}(l)$.) Thus, every result we obtain for linear sandwich semigroups leads to analogous results for the semigroups $\mathcal{R}_{k}(l)$ and $\mathcal{C}_{k}(l)$. For example, we deduce from Theorem 4.14 that $\operatorname{rank}\left(\mathcal{C}_{k}(l)\right)=\operatorname{rank}\left(\mathcal{R}_{k}(l)\right)=\left[\begin{array}{l}k \\ l\end{array}\right]_{q}$ if $|\mathbb{F}|=q<\infty$. Note that the sandwich semigroups $\mathcal{M}_{m n}^{J}$ pictured in Figure 5 satisfy $r=\min (m, n)$, so Figure 5 essentially pictures eggbox diagrams of $\mathcal{C}_{3}(2)$ and $\mathcal{R}_{4}(2)$.

Remark 5.4. Similarly, one may think of an arbitrary linear sandwich semigroup $\mathcal{M}_{m n}^{J}$ itself as a (nonsandwich) matrix semigroup, as noted by Thrall in 92 and slightly adapted as follows. Consider the set $\mathscr{M}$ of all $(m+n-r) \times(m+n-r)$ matrices that may be written in $3 \times 3$ block form $\left[\begin{array}{lll}O & O & O \\ B & A & O \\ D & C & O\end{array}\right]$, where $A \in \mathcal{M}_{r}, D \in \mathcal{M}_{m-r, n-r}$ (and from which the dimensions of the other sub-matrices may be derived). One easily checks that the matrices from $\mathscr{M}$ multiply according to the rule $\left[\begin{array}{ccc}O & O & O \\ B & A & O \\ D & C & O\end{array}\right]\left[\begin{array}{ccc}O & O & O \\ F & E & O \\ H & G & O\end{array}\right]=\left[\begin{array}{ccc}A & O & O \\ C F & C E & O \\ C & 0\end{array}\right]$, so that $\left[\begin{array}{ll}A & B \\ C & D\end{array}\right] \mapsto\left[\begin{array}{ccc}O & O & O \\ B & A & O \\ D & C & O\end{array}\right]$ determines an isomorphism $\left(\mathcal{M}_{m n}, \star_{J}\right) \rightarrow(\mathscr{M}, \cdot)$. Note also that

$$
\mathscr{M}=\mathcal{R}_{m+n-r}^{*}(m) \cap \mathcal{C}_{m+n-r}(n)
$$

where here we write $\mathcal{R}_{k}^{*}(l)=\left\{X \in \mathcal{M}_{k}: \mathfrak{r}_{1}(X)=\cdots=\mathfrak{r}_{k-l}(X)=O\right\}$. (It is easily seen that the map $\left[\begin{array}{ll}A & B \\ O & O\end{array}\right] \rightarrow\left[\begin{array}{ll}O & O \\ B & A\end{array}\right]$ determines an isomorphism $\mathcal{R}_{k}(l) \rightarrow \mathcal{R}_{k}^{*}(l)$.) Since using this isomorphic copy $\mathscr{M}$ of $\mathcal{M}_{m n}^{J}$ does not appear to confer any obvious advantage, we will make no further reference to it.

The regular elements of $\mathcal{C}_{k}(l)$ and $\mathcal{R}_{k}(l)$ (and also of $\mathcal{I}_{k}(l)$ and $\mathcal{K}_{k}(l)$ ) were classified in [72]. The next result, which gives a much simpler description of these regular elements, may be deduced from 72 , Theorems 3.4 and 3.8] (and vice versa), but we include a simple proof for convenience.

Proposition 5.5. The regular elements of the semigroups $\mathcal{C}_{m}(r)=\mathcal{M}_{m n} J$ and $\mathcal{R}_{n}(r)=J \mathcal{M}_{m n}$ are given by

$$
\begin{aligned}
& \operatorname{Reg}\left(\mathcal{C}_{m}(r)\right)=\operatorname{Reg}\left(\mathcal{M}_{m n} J\right)=P J=\left\{X \in \mathcal{M}_{m n} J: \operatorname{rank}(J X)=\operatorname{rank}(X)\right\} \\
& \operatorname{Reg}\left(\mathcal{R}_{n}(r)\right)=\operatorname{Reg}\left(J \mathcal{M}_{m n}\right)=J P=\left\{X \in J \mathcal{M}_{m n}: \operatorname{rank}(X J)=\operatorname{rank}(X)\right\}
\end{aligned}
$$

Proof. We just prove the second statement as the other is dual. Let $X=\left[\begin{array}{cc}A & B \\ C\end{array}\right] \in \mathcal{M}_{m n}$, and put $X^{\prime}=\left[\begin{array}{ll}A & B \\ O & O\end{array}\right] \in \mathcal{M}_{m n}$. Then $J X=J X^{\prime}=\left[\begin{array}{ll}A & B \\ O & O\end{array}\right] \in \mathcal{M}_{n}$ (where the zero matrices in the last expression have $n-r$ rows). Since $X^{\prime}$ clearly belongs to $P_{2}$ (by Proposition 4.3), we have $J \mathcal{M}_{m n} \subseteq J P_{2}$. Next, note that $K J=J_{m m r}$, so that $K J Y=Y$ for all $Y \in \mathcal{M}_{m n}$ of the form $Y=\left[\begin{array}{ll}A & B \\ O & O\end{array}\right]$. Now suppose $X \in \mathcal{M}_{m n}$ is such that $J X \in \operatorname{Reg}\left(J \mathcal{M}_{m n}\right)$. As above, we may assume that $X=\left[\begin{array}{cc}A & B \\ O & O\end{array}\right]$. So $(J X)=(J X)(J Y)(J X)$ for some $Y \in \mathcal{M}_{m n}$. But then $X=K(J X)=K(J X J Y J X)=X J Y J X=X \star Y \star X$, so that, in fact, 
$X \in \operatorname{Reg}\left(\mathcal{M}_{m n}^{J}\right)=P$. This completes the proof that $\operatorname{Reg}\left(J \mathcal{M}_{m n}\right) \subseteq J P$. The reverse inclusion is easily checked.

Now suppose $X=J Y$ where $Y=\left[\begin{array}{cc}A & A N \\ O & O\end{array}\right] \in P$. Then $X=J Y=\left[\begin{array}{cc}A & A N \\ O & O\end{array}\right]$ (with appropriately sized zero matrices), so $\operatorname{rank}(X)=\operatorname{rank}(J Y)=\operatorname{rank}(Y)=\operatorname{rank}(J Y J)=\operatorname{rank}(X J)$, where we have used Proposition 4.3. Conversely, suppose $X \in J \mathcal{M}_{m n}$ is such that $\operatorname{rank}(X J)=\operatorname{rank}(X)$. As before, we may assume that $X=J Y$ where $Y \in P_{2}$. Then $\operatorname{rank}(Y)=\operatorname{rank}(J Y)=\operatorname{rank}(X)=\operatorname{rank}(X J)=\operatorname{rank}(J Y J)$, so that $Y \in P$. This completes the proof.

Remark 5.6. As always, the condition $\operatorname{rank}(J X)=\operatorname{rank}(X)$, for $X \in \mathcal{M}_{m n}$, is equivalent to saying that rows $\mathfrak{r}_{r+1}(X), \ldots, \mathfrak{r}_{m}(X)$ belong to $\operatorname{span}\left\{\mathfrak{r}_{1}(X), \ldots, \mathfrak{r}_{r}(X)\right\}$, with a dual statement holding for the condition $\operatorname{rank}(X J)=\operatorname{rank}(X)$. The regular elements of the corresponding semigroups of linear transformations are given by

$$
\begin{aligned}
\operatorname{Reg}\left(\mathcal{K}_{m}(r)\right) & =\left\{\alpha \in \mathcal{K}_{m}(r): \operatorname{im}(\alpha) \cap W_{m r}^{\perp}=\{0\}\right\}, \\
\operatorname{Reg}\left(\mathcal{I}_{n}(r)\right) & =\left\{\alpha \in \mathcal{I}_{n}(r): \operatorname{im}(\alpha)=\alpha\left(W_{n r}\right)\right\} .
\end{aligned}
$$

Putting together all the above, we have proved the following. (In the following statement, we slightly abuse notation by still denoting the map $\mathcal{C}_{m}(r)=\mathcal{M}_{m n} J \rightarrow \mathcal{M}_{r}$ by $\Phi_{1}$ and so on.)

Theorem 5.7. We have the following commutative diagrams of semigroup epimorphisms:
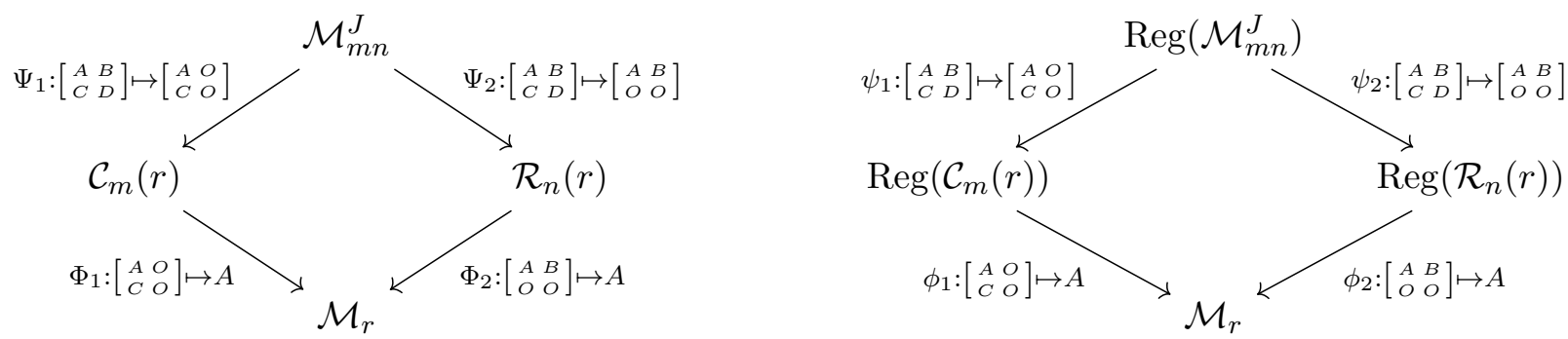

The remaining results of this section concern the regular subsemigroup $P=\operatorname{Reg}\left(\mathcal{M}_{m n}^{J}\right)$. From now on, we denote by $\phi=\phi_{1} \circ \psi_{1}=\phi_{2} \circ \psi_{2}$ the induced epimorphism $\phi: P \rightarrow \mathcal{M}_{r}$. Also, for $X=\left[\begin{array}{cc}A & B \\ C\end{array}\right] \in P$, we write $\bar{X}=\phi(X)=A$. The next result shows how the second commutative diagram from Theorem 5.7 may be used to identify $\operatorname{Reg}\left(\mathcal{M}_{m n}^{J}\right)$ as a special kind of subdirect product of $\operatorname{Reg}\left(\mathcal{C}_{m}(r)\right)$ and $\operatorname{Reg}\left(\mathcal{R}_{n}(r)\right)$.

Proposition 5.8. There is an embedding

$$
\psi: \operatorname{Reg}\left(\mathcal{M}_{m n}^{J}\right) \rightarrow \operatorname{Reg}\left(\mathcal{C}_{m}(r)\right) \times \operatorname{Reg}\left(\mathcal{R}_{n}(r)\right): X \mapsto\left(\psi_{1}(X), \psi_{2}(X)\right)=(X J, J X) .
$$

As such, $P=\operatorname{Reg}\left(\mathcal{M}_{m n}^{J}\right)$ is (isomorphic to) a pullback product of $P J=\operatorname{Reg}\left(\mathcal{C}_{m}(r)\right)$ and $J P=\operatorname{Reg}\left(\mathcal{R}_{n}(r)\right)$. Namely,

$$
P \cong \operatorname{im}(\psi)=\left\{\left(\psi_{1}(X), \psi_{2}(X)\right): X \in P\right\}=\left\{(Y, Z) \in P J \times J P: \phi_{1}(Y)=\phi_{2}(Z)\right\} .
$$

Proof. Clearly, $\psi$ is a homomorphism. Now let $X=[M, A, N]$ and $Y=[K, B, L]$ be elements of $P$ with $\psi(X)=\psi(Y)$. Then

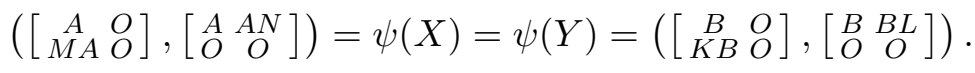

Comparing various coordinates, we deduce $A=B, M A=K B$ and $A N=B L$, giving $X=\left[\begin{array}{c}A \\ M A\end{array} \stackrel{A N}{M A N}\right]=$ $\left[\begin{array}{cc}B & B L \\ K B & K B L\end{array}\right]=Y$, completing the proof that $\psi$ is injective.

To prove the statement about $\operatorname{im}(\psi)$, let $X \in P$ and put $Y=\psi_{1}(X)=X J$ and $Z=\psi_{2}(X)=J X$. Then $\phi_{1}(Y)=J Y=J X J=Z J=\phi_{2}(Z)$. Conversely, suppose $(Y, Z) \in P J \times J P$ satisfies $J Y=Z J$. Say $Y=U J$ and $Z=J V$, where $U=[M, A, N]$ and $V=[K, B, L]$ belong to $P$. Then $J Y=J U J=\left[\begin{array}{l}A \\ O\end{array} O_{O}\right]$ and $Z J=J V J=\left[\begin{array}{ll}B & O \\ O & O\end{array}\right]$, giving $A=B$. But then $(Y, Z)=\psi(X)$, where $X=[M, A, L] \in P$. 
Remark 5.9. We note that the previous result does not lift to a similar identification of $\mathcal{M}_{m n}^{J}$ as a pullback product of $\mathcal{C}_{m}(r)$ and $\mathcal{R}_{n}(r)$ because the induced map

$$
\Psi: \mathcal{M}_{m n}^{J} \rightarrow \mathcal{C}_{m}(r) \times \mathcal{R}_{n}(r): X \mapsto\left(\Psi_{1}(X), \Psi_{2}(X)\right)=(X J, J X)
$$

is not injective. Indeed, if $X=\left[\begin{array}{cc}A & B \\ C & D\end{array}\right] \in \mathcal{M}_{m n}$, then $\Psi(X)=\left(\left[\begin{array}{ll}A & O \\ C & O\end{array}\right],\left[\begin{array}{ll}A & B \\ O & O\end{array}\right]\right)$, with $\left[\begin{array}{cc}A & B \\ C\end{array}\right]$ mapping to the same pair for any other $E \in \mathcal{M}_{m-r, n-r}$.

Remark 5.10. More generally, given a partial semigroup $(S, \cdot, I, \lambda, \rho)$, the epimorphisms $\Psi_{1}$ and $\Psi_{2}$ from Theorem 2.15(v) allow for the definition of a map

$$
\Psi:\left(S_{i j}, \star_{a}\right) \rightarrow\left(S_{i j} a, \cdot\right) \times\left(a S_{i j}, \cdot\right): x \mapsto(x a, a x) .
$$

To say that $\Psi$ is injective is to say that, for all $x, y \in S_{i j}, x a=y a$ and $a x=a y$ together imply $x=y$. Compare this to the notion of a weakly reductive semigroup $S$, in which, for every $x, y \in S$, the assumption that $x a=y a$ and $a x=a y$ for all $a \in S$ implies $x=y$. See for example [71, Definition 1.42].

We conclude this section with a simple but important observation that shows that $P=\operatorname{Reg}\left(\mathcal{M}_{m n}^{J}\right)$ is a homomorphic image of the direct product of a rectangular band by the (non-sandwich) matrix semigroup $\mathcal{M}_{r}$. (Recall that a rectangular band is a semigroup of the form $S \times T$ with product $\left(s_{1}, t_{1}\right)\left(s_{2}, t_{2}\right)=\left(s_{1}, t_{2}\right)$.) Its proof is routine, relying on Proposition 4.3 and the rule $[M, A, N] \star[K, B, L]=[M, A B, L]$. For the statement, recall that the kernel of a semigroup homomorphism $\phi: S \rightarrow T$ (not to be confused with the kernel of a linear transformation) is the congruence $\operatorname{ker}(\phi)=\{(x, y) \in S \times S: \phi(x)=\phi(y)\}$. (A congruence on a semigroup $S$ is an equivalence relation $\sim$ for which $x_{1} \sim y_{1}$ and $x_{2} \sim y_{2}$ together imply $x_{1} x_{2} \sim y_{1} y_{2}$ for all $x_{1}, x_{2}, y_{1}, y_{2} \in S$; the quotient $S / \sim$ of all $\sim$-classes is a semigroup under the induced operation. The first homomorphism theorem for semigroups states that any semigroup homomorphism $\phi: S \rightarrow T$ induces an isomorphism $S / \operatorname{ker}(\phi) \cong \operatorname{im}(\phi)$.)

Proposition 5.11. Consider the semigroup $U=\mathcal{M}_{m-r, r} \times \mathcal{M}_{r} \times \mathcal{M}_{r, n-r}$ under the operation $\diamond$ defined by

$$
(M, A, N) \diamond(K, B, L)=(M, A B, L) .
$$

Define an equivalence $\sim$ on $U$ by

$$
(M, A, N) \sim(K, B, L) \Leftrightarrow A=B, M A=K B \text { and } A N=B L .
$$

Then $\sim$ is a congruence on $U$, and the map

$$
\xi: U \rightarrow P=\operatorname{Reg}\left(\mathcal{M}_{m n}^{J}\right):(M, A, N) \mapsto[M, A, N]=\left[\begin{array}{cc}
A & A N \\
M A & M A N
\end{array}\right]
$$

is an epimorphism with $\operatorname{ker}(\xi)=\sim$. In particular, $P \cong U / \sim$.

\section{The regular subsemigroup}

In this section, we continue to study the subsemigroup $P=\operatorname{Reg}\left(\mathcal{M}_{m n}^{J}\right)$ consisting of all regular elements of $\mathcal{M}_{m n}^{J}$. Eggbox diagrams of $P=\operatorname{Reg}\left(\mathcal{M}_{43}^{J}\left(\mathbb{Z}_{2}\right)\right)$ are given in Figure 8 for values of $0 \leq \operatorname{rank}(J) \leq 3$; more examples can be seen by inspecting the regular $\mathscr{D}^{J}$-classes in Figures 4 and 5 . Comparing Figure 8 with Figure 3, which pictures the full linear monoids $\mathcal{M}_{r}\left(\mathbb{Z}_{2}\right)$ for $0 \leq r \leq 3$, an interesting pattern seems to emerge: namely, that $P=\operatorname{Reg}\left(\mathcal{M}_{43}^{J}\left(\mathbb{Z}_{2}\right)\right)$ appears to be a kind of "inflation" of $\mathcal{M}_{r}$, where $r=\operatorname{rank}(J)$. One of the goals of this section is to explain this phenomenon, and we do so by further exploring the map

$$
\phi: P \rightarrow \mathcal{M}_{r}: X=[M, A, N] \mapsto \bar{X}=A
$$

defined after Theorem 5.7. We also calculate $|P|, \operatorname{rank}(P)$, and the number and sizes of various Green's classes. As before, we assume that $J=J_{n m r}=\left[\begin{array}{cc}I_{r} & O \\ O & O\end{array}\right] \in \mathcal{M}_{n m}$. Since $\mathcal{M}_{m n}^{J}$ is just a zero semigroup if $r=0$, we generally assume that $r \geq 1$. 

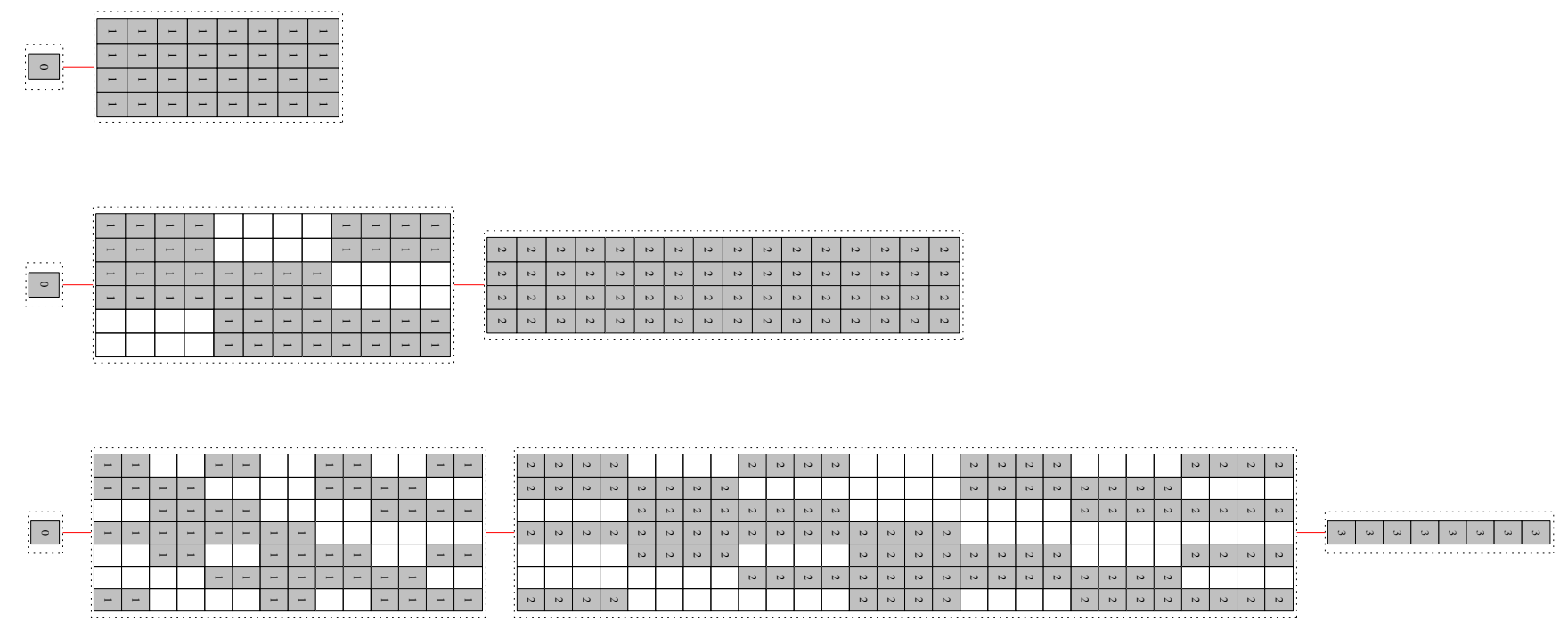

Figure 8: Egg box diagrams (drawn sideways) of the regular linear sandwich semigroups $P=\operatorname{Reg}\left(\mathcal{M}_{43}^{J}\left(\mathbb{Z}_{2}\right)\right)$, where $\operatorname{rank}(J)=0,1,2,3$ (top to bottom).

Now, Theorem 4.5 enables us to immediately describe Green's relations on $P=\operatorname{Reg}\left(\mathcal{M}_{m n}^{J}\right)$. Since $P$ is a regular subsemigroup of $\mathcal{M}_{m n}^{J}$, the $\mathscr{R}, \mathscr{L}, \mathscr{H}$ relations on $P$ are just the restrictions of the corresponding relations on $\mathcal{M}_{m n}^{J}$ (see for example 40,44$]$ ), and it is easy to check that this is also true for the $\mathscr{D}=\mathscr{J}$ relation in this case. So if $X \in P$ and $\mathscr{K}$ is one of $\mathscr{R}, \mathscr{L}, \mathscr{H}, \mathscr{D}$, we will continue to write $\mathscr{K}^{J}$ for the $\mathscr{K}$ relation on $P$, and $K_{X}^{J}$ for the $\mathscr{K}^{J}$-class of $X$ in $P$. Parts (i-iv) of the next result also appear in [9, Theorem 2.3].

Corollary 6.1. If $X \in P$, then

(i) $R_{X}^{J}=R_{X} \cap P=\{Y \in P: \operatorname{Col}(X)=\operatorname{Col}(Y)\}$,

(ii) $L_{X}^{J}=L_{X} \cap P=\{Y \in P: \operatorname{Row}(X)=\operatorname{Row}(Y)\}$,

(iii) $H_{X}^{J}=H_{X} \cap P=H_{X}=\{Y \in P: \operatorname{Col}(X)=\operatorname{Col}(Y)$ and $\operatorname{Row}(X)=\operatorname{Row}(Y)\}$,

(iv) $D_{X}^{J}=D_{X} \cap P=\{Y \in P: \operatorname{rank}(X)=\operatorname{rank}(Y)\}$.

The $\mathscr{D}^{J}$-classes of $P$ form a chain: $D_{0}^{J}<\cdots<D_{r}^{J}$, where $D_{s}^{J}=\{X \in P: \operatorname{rank}(X)=s\}$ for each $0 \leq s \leq r$.

Also, the regularity of $P$ means that $P$ inherits the stability property from $\mathcal{M}_{m n}^{J}$. The next result gives some combinatorial information about the size of $P$, and of various Green's classes in $P$, in the case that $\mathbb{F}$ is finite. Recall that $\left\{e_{k 1}, \ldots, e_{k k}\right\}$ is the standard basis of $V_{k}=\mathbb{F}^{k}$ and that $W_{k s}=\operatorname{span}\left\{e_{k 1}, \ldots, e_{k s}\right\}$ for each $0 \leq s \leq k$.

Proposition 6.2. Suppose $|\mathbb{F}|=q<\infty$. Let $X \in P$ with $\operatorname{rank}(X)=s$. Then

(i) $\left|R_{X}^{J}\right|=q^{s(n-r)} q^{\left(\begin{array}{c}s \\ 2\end{array}\right)}(q-1)^{s}[s]_{q} !\left[\begin{array}{l}r \\ s\end{array}\right]_{q}$,

(ii) $\left|L_{X}^{J}\right|=q^{s(m-r)} q^{\left(\begin{array}{c}s \\ 2\end{array}\right)}(q-1)^{s}[s]_{q} !\left[\begin{array}{l}r \\ s\end{array}\right]_{q}$,

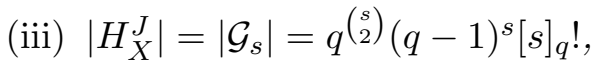

(iv) $D_{X}^{J}=D_{s}^{J}$ is the union of: 

(a) $q^{s(m-r)}\left[\begin{array}{l}r \\ s\end{array}\right]_{q} \mathscr{R}^{J}$-classes,
(b) $q^{s(n-r)}\left[\begin{array}{l}r \\ s\end{array}\right]_{q} \mathscr{L}^{J}$-classes,
(c) $q^{s(m+n-2 r)}\left[\begin{array}{c}r \\ s\end{array}\right]_{q}^{2} \mathscr{H}^{J}$-classes,

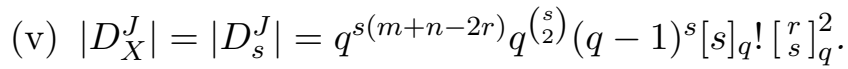

Consequently, $|P|=\left|\operatorname{Reg}\left(\mathcal{M}_{m n}^{J}\right)\right|=\sum_{s=0}^{r} q^{s(m+n-2 r)} q^{\left(\begin{array}{c}s \\ 2\end{array}\right)}(q-1)^{s}[s]_{q} !\left[\begin{array}{c}r \\ s\end{array}\right]_{q}^{2}$.

Proof. We start with (i). Since $\left|R_{X}^{J}\right|=\left|R_{Y}^{J}\right|$ for all $Y \in D_{X}^{J}=D_{s}^{J}$, we may assume $X=J_{m n s}$. Now, $\operatorname{Col}(X)=W_{m s}$. By Proposition 4.3 and Corollary 6.1, we have

$$
\begin{aligned}
R_{X}^{J} & =\left\{Y \in P: Y \mathscr{R}^{J} X\right\} \\
& =\left\{Y \in \mathcal{M}_{m n}: \operatorname{Col}(X)=\operatorname{Col}(Y), \operatorname{Col}(Y)=\operatorname{Col}(Y J), \operatorname{Row}(Y)=\operatorname{Row}(J Y)\right\} \\
& =\left\{Y \in \mathcal{M}_{m n}: \operatorname{Col}(Y)=\operatorname{Col}(Y J)=W_{m s}\right\},
\end{aligned}
$$

since if $\operatorname{Col}(Y)=W_{m s}$, then $Y$ is of the form $Y=\left[\begin{array}{cc}A & B \\ O_{m-s, r} & O_{m-s, n-r}\end{array}\right]$ for some $A \in \mathcal{M}_{s r}$ and $B \in \mathcal{M}_{s, n-r}$, in which case $J Y=\left[\begin{array}{cc}A & B \\ O_{n-s, r} & O_{n-s, n-r}\end{array}\right]$ automatically has the same row space as $Y$.

Now consider some $Y \in R_{X}^{J}$. As noted above, we must have $Y=\left[\begin{array}{cc}A & B \\ O & O\end{array}\right]$ for some $A \in \mathcal{M}_{s r}$ and $B \in \mathcal{M}_{s, n-r}$. Since $Y J=\left[\begin{array}{cc}A & O \\ O & O\end{array}\right]$, the condition $\operatorname{Col}(Y J)=W_{m s}$ is equivalent to $\operatorname{Col}(A)=V_{s}$. In particular, there is no restriction on the entries of $B$, so $B$ may be chosen (arbitrarily, and independently of $A$ ) in $q^{s(n-r)}$ ways. Also, $\operatorname{dim}(\operatorname{Row}(A))=\operatorname{dim}(\operatorname{Col}(A))=s$. So $A$ may be specified by listing its rows (in order), which are $s$ linearly independent row vectors from $\mathbb{F}^{r}$. The number of possible choices for $A$ is therefore $\left(q^{r}-1\right)\left(q^{r}-q\right) \cdots\left(q^{r}-q^{s-1}\right)=q^{\left(\begin{array}{c}s \\ 2\end{array}\right)}(q-1)^{s}[s]_{q} !\left[\begin{array}{l}r \\ s\end{array}\right]_{q}$. Multiplying these two values gives (i).

Part (ii) is dual to (i). Part (iii) follows directly from Corollary 6.1(iii) and Lemma 3.4(iii). Parts (a) and (b) of (iv) follow by dividing $\left|L_{X}^{J}\right|$ and $\left|R_{X}^{J}\right|$ by $\left|H_{X}^{J}\right|$, respectively. Part (c) follows from (a) and (b). Part (v) follows from (iii) and part (c) of (iv). The formula for $|P|$ is obtained by adding the sizes of the $\mathscr{D}^{J}$-classes.

Recall that, for $X=[M, A, N] \in P$, we write $\bar{X}=\phi(X)=A \in \mathcal{M}_{r}$. We extend this notation to subsets of $P$, so if $\Omega \subseteq P$, we write $\bar{\Omega}=\{\bar{X}: X \in \Omega\}$. We now show how the epimorphism $\phi: P \rightarrow \mathcal{M}_{r}$ may be used to relate Green's relations on the semigroups $P$ and $\mathcal{M}_{r}$. If $X, Y \in P$ and $\mathscr{K}$ is one of $\mathscr{R}, \mathscr{L}, \mathscr{H}, \mathscr{D}$, we say $X \widehat{\mathscr{K}}^{J} Y$ if $\bar{X} \mathscr{K} \bar{Y}$ (in $\mathcal{M}_{r}$ ). Denote by $\widehat{K}_{X}^{J}=\phi^{-1}\left(K_{\bar{X}}\right)=\left\{Y \in P: X \widehat{\mathscr{K}}^{J} Y\right\}$ the $\widehat{\mathscr{K}}^{J}$-class of $X$ in $P$. We first need a technical result.

Lemma 6.3. Let $X, Y \in P$. If $X \widehat{\mathscr{D}}^{J} Y$, then $\left|\widehat{R}_{X}^{J}\right|=\left|\widehat{R}_{Y}^{J}\right|$ and $\left|\widehat{L}_{X}^{J}\right|=\left|\widehat{L}_{Y}^{J}\right|$.

Proof. By duality, it suffices to prove the statement about $\widehat{\mathscr{R}}^{J}$-classes. Now, $X \widehat{\mathscr{D}}^{J} Y$ means that $X \widehat{\mathscr{L}}^{J} W \widehat{\mathscr{R}}^{J} Y$ for some $W \in P$. Since $\widehat{R}_{Y}^{J}=\widehat{R}_{W}^{J}$, we may assume without loss of generality that $X \widehat{\mathscr{L}}^{J} Y$. Write $X=[M, A, N]$ and $Y=[K, B, L]$. By definition $X \widehat{\mathscr{L}}^{J} Y$, means that $A \mathscr{L} B$ in $\mathcal{M}_{r}$, so $A=U B$ for some $U \in \mathcal{G}_{r}$ by Lemma 3.3. Now let $Z=\left[M^{\prime}, A^{\prime}, N^{\prime}\right] \in \widehat{R}_{X}^{J}$, and define $\alpha(Z)=\left[M^{\prime} U, U^{-1} A^{\prime}, N^{\prime}\right]$. It is easy to check that for any other representation $Z=\left[M^{\prime \prime}, A^{\prime \prime}, N^{\prime \prime}\right]$, we have $\left[M^{\prime \prime} U, U^{-1} A^{\prime \prime}, N^{\prime \prime}\right]=\left[M^{\prime} U, U^{-1} A^{\prime}, N^{\prime}\right]$, so that $\alpha(Z)$ is well-defined. Also,

$$
Z \widehat{\mathscr{R}}^{J} X \Rightarrow A^{\prime} \mathscr{R} A \Rightarrow U^{-1} A^{\prime} \mathscr{R} U^{-1} A=B \Rightarrow \alpha(Z) \widehat{\mathscr{R}}^{J} Y .
$$

Thus $\alpha$ is a map $\alpha: \widehat{R}_{X}^{J} \rightarrow \widehat{R}_{Y}^{J}$. It is easy to check that $\widehat{R}_{Y}^{J} \rightarrow \widehat{R}_{X}^{J}:\left[M^{\prime}, A^{\prime}, N^{\prime}\right] \mapsto\left[M^{\prime} U^{-1}, U A^{\prime}, N^{\prime}\right]$ is the inverse mapping of $\alpha$. We conclude that $\left|\widehat{R}_{X}^{J}\right|=\left|\widehat{R}_{Y}^{J}\right|$.

For the proof of the next result, we note that stability of $\mathcal{M}$ implies that $A^{2} \mathscr{D} A \Leftrightarrow A^{2} \mathscr{H} A$ for all $A \in \mathcal{M}_{r}$. We also use the fact that an $\mathscr{H}$-class $H$ of a semigroup $S$ is a group if and only if $x^{2} \in H$ for some (and hence for all) $x \in H$ [44, Theorem 2.2.5]. Recall that a $k \times l$ rectangular band is a semigroup of the form $S \times T$ with product $\left(s_{1}, t_{1}\right)\left(s_{2}, t_{2}\right)=\left(s_{1}, t_{2}\right)$, where $|S|=k$ and $|T|=l$. A $k \times l$ rectangular group with respect to a group $G$ is a direct product of a $k \times l$ rectangular band with $G$. 
For the proof of the next result (and elsewhere), it will be convenient to define a number of equivalence relations. For $A \in \mathcal{M}_{r}$, we define equivalences $\sim_{A}$ and $\approx_{A}$ on $\mathcal{M}_{m-r, r}$ and $\mathcal{M}_{r, n-r}$ (respectively) by

$$
M_{1} \sim_{A} M_{2} \Leftrightarrow M_{1} A=M_{2} A \quad \text { and } \quad N_{1} \approx_{A} N_{2} \Leftrightarrow A N_{1}=A N_{2} .
$$

Theorem 6.4. Suppose $|\mathbb{F}|=q<\infty$. Let $X \in P=\operatorname{Reg}\left(\mathcal{M}_{m n}^{J}\right)$ and put $s=\operatorname{rank}(X)$.

(i) $\widehat{R}_{X}^{J}$ is the union of $q^{s(m-r)} \mathscr{R}^{J}$-classes of $P$.

(ii) $\widehat{L}_{X}^{J}$ is the union of $q^{s(n-r)} \mathscr{L}^{J}$-classes of $P$.

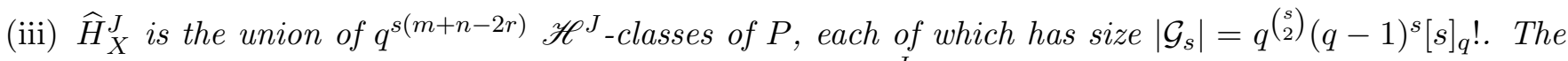
map $\phi: P \rightarrow \mathcal{M}_{r}$ is injective when restricted to any $\mathscr{H}^{J}$-class of $P$.

(iv) If $H_{\bar{X}}$ is a non-group $\mathscr{H}$-class of $\mathcal{M}_{r}$, then each $\mathscr{H}^{J}$-class of $P$ contained in $\widehat{H}_{X}^{J}$ is a non-group.

(v) If $H_{\bar{X}}$ is a group $\mathscr{H}$-class of $\mathcal{M}_{r}$, then each $\mathscr{H}^{J}$-class of $P$ contained in $\widehat{H}_{X}^{J}$ is a group isomorphic to $\mathcal{G}_{s}$; further, $\hat{H}_{X}^{J}$ is a $q^{s(m-r)} \times q^{s(n-r)}$ rectangular group with respect to $\mathcal{G}_{s}$.

(vi) $\widehat{\mathscr{D}}^{J}=\mathscr{D}^{J}$ and $\widehat{D}_{X}^{J}=D_{X}^{J}=D_{s}^{J}=\{Y \in P: \operatorname{rank}(Y)=s\}$ is the union of:

(a) $\left[\begin{array}{l}r \\ s\end{array}\right]_{q} \widehat{\mathscr{R}}^{J}$-classes (and the same number of $\widehat{\mathscr{L}}^{J}$-classes) of $P$,

(b) $q^{s(m-r)}\left[\begin{array}{l}r \\ s\end{array}\right]_{q} \mathscr{R}^{J}$-classes of $P$,

(b) $q^{s(n-r)}\left[\begin{array}{l}r \\ s\end{array}\right]_{q} \mathscr{L}^{J}$-classes of $P$,

(d) $\left[\begin{array}{l}r \\ s\end{array}\right]_{q}^{2} \widehat{\mathscr{H}}^{J}$-classes of $P$,

(e) $q^{s(m+n-2 r)}\left[\begin{array}{l}r \\ s\end{array}\right]_{q}^{2} \mathscr{H}^{J}$-classes of $P$.

Proof. First observe that if $\rho: S \rightarrow T$ is an epimorphism of semigroups, and if $K$ is a $\mathscr{K}$-class of $T$ where $\mathscr{K}$ is one of $\mathscr{R}, \mathscr{L}, \mathscr{H}$, then $\rho^{-1}(K)$ is a union of $\mathscr{K}$-classes of $S$. Throughout the proof, we write

$$
X=[M, A, N]=\left[\begin{array}{cc}
A & A N \\
M A & M A N
\end{array}\right]
$$

so $A \in \mathcal{M}_{r}$ satisfies $\operatorname{rank}(A)=\operatorname{rank}(J X J)=\operatorname{rank}(X)=s$. We note that $\widehat{\mathscr{D}}^{J}=\mathscr{D}^{J}$ immediately follows.

(i) By the first observation, it suffices to count the number of $\mathscr{R}^{J}$-classes contained in $\widehat{R}_{X}^{J}$. Since $\left|\widehat{R}_{X}^{J}\right|=$ $\left|\widehat{R}_{Y}^{J}\right|$ for all $Y \in D_{s}^{J}$ by Lemma 6.3 , it follows that each $\widehat{\mathscr{R}}^{J}$-class of $D_{s}^{J}$ contains the same number of $\mathscr{R}^{J}$-classes. By Lemma 3.4, $D_{s}\left(\mathcal{M}_{r}\right)$ is the union of $\left[\begin{array}{l}r \\ s\end{array}\right]_{q} \mathscr{R}$-classes (and the same number of $\mathscr{L}$ classes), so it follows that $\bar{D}_{s}^{J}$ is the union of $\left[\begin{array}{l}r \\ s\end{array}\right]_{q} \widehat{\mathscr{R}}^{J}$-classes (and the same number of $\widehat{\mathscr{L}}^{J}$-classes). By Proposition 6.2, $D_{s}^{J}$ is the union of $q^{s(m-r)}\left[\begin{array}{c}r \\ s\end{array}\right]_{q} \mathscr{R}^{J}$-classes. Dividing these, it follows that each $\widehat{\mathscr{R}}^{J}$-class of $D_{s}^{J}$ is the union of $q^{s(m-r)} \mathscr{R}^{J}$-classes.

(ii) This is dual to (i).

(iii) The statement concerning the number of $\mathscr{H}^{J}$-classes contained in $\widehat{H}_{X}^{J}$ follows immediately from (i) and (ii), and the size of these $\mathscr{H}^{J}$-classes was given in Proposition 6.2. Next, for any $B \in \mathcal{M}_{r}$ with $B \mathscr{H} A$, it is easy to check that $[M, B, N] \mathscr{H}^{J} X$. So the set $\Omega=\left\{[M, B, N]: B \in H_{A}\right\}$ is contained in $\mathscr{H}_{X}^{J}$. Since $|\Omega|=\left|H_{A}\right|=\left|\mathcal{G}_{s}\right|=\left|H_{X}^{J}\right|$, we see that $H_{X}^{J}=\Omega$. For any $Z=[M, B, N] \in \Omega=H_{X}^{J}$, we have $\phi(Z)=B$, so it follows that $\left.\phi\right|_{H_{X}^{J}}$ is injective.

(iv) Suppose $H_{A}=H_{\bar{X}}$ is a non-group $\mathscr{H}$-class of $\mathcal{M}_{r}$, and let $Y=[K, B, L] \in \widehat{H}_{X}^{J}$ be arbitrary. Since $Y \hat{\mathscr{H}}^{J} X$, it follows that $B=\bar{Y} \mathscr{H} \bar{X}=A$. Since $H_{B}=H_{A}$ is not a group, we have $B^{2} \notin H_{B}$, whence $B^{2} \notin D_{B}$ and $\operatorname{rank}\left(B^{2}\right)<\operatorname{rank}(B)=\operatorname{rank}(A)=s$. But then $Y^{2}=\left[K, B^{2}, L\right] \notin D_{s}^{J}=D_{Y}^{J}$, so that $Y^{2} \notin H_{Y}^{J}$, and we conclude that $H_{Y}^{J}$ is not a group. 
(v) Suppose $H_{\bar{X}}$ is a group. Then $\bar{Y}^{2} \in H_{\bar{X}}$ for any $Y \in \widehat{H}_{X}^{J}$, so $\operatorname{rank}(Y \star Y)=\operatorname{rank}\left(\bar{Y}^{2}\right)=\operatorname{rank}(\bar{Y})=$ $\operatorname{rank}(Y)$, giving $Y \star Y \mathscr{D}^{J} Y$, so that $Y \star Y \in H_{Y}^{J}$ and $H_{Y}^{J}$ is a group. By (iii), the restriction of $\phi$ to $H_{Y}^{J}$ yields an isomorphism onto $H_{\bar{Y}} \cong \mathcal{G}_{s}$.

Let $E \in \mathcal{M}_{r}$ be the identity element of the group $H_{A}$. Let $\mathscr{M}_{E} \subseteq \mathcal{M}_{m-r, r}$ (resp., $\mathscr{N}_{E} \subseteq \mathcal{M}_{r, n-r}$ ) be a cross-section of the $\sim_{E}$-classes (resp., $\approx_{E}$-classes) in $\mathcal{M}_{m-r, r}$ (resp., $\mathcal{M}_{r, n-r}$ ). It is easy to check that every $Y \in \widehat{H}_{X}^{J}$ may be uniquely represented as $Y=[K, B, L]$ for some $K \in \mathscr{M}_{E}, B \in H_{A}$ and $L \in \mathscr{N}_{E}$. It follows that the map

$$
\mathscr{M}_{E} \times H_{A} \times \mathscr{N}_{E} \rightarrow \widehat{H}_{A}^{J}:(K, B, L) \mapsto[K, B, L]
$$

is a well-defined isomorphism, where the (rectangular group) product on $\mathscr{M}_{E} \times H_{A} \times \mathscr{N}_{E}$ is defined by $\left(K_{1}, B_{1}, L_{1}\right) \cdot\left(K_{2}, B_{2}, L_{2}\right)=\left(K_{1}, B_{1} B_{2}, L_{2}\right)$. We have already observed that $H_{A} \cong \mathcal{G}_{s}$, and the dimensions of the rectangular band $\mathscr{M}_{E} \times \mathscr{N}_{E}$ follow from parts (i-iii) together with the observation that $\left\{[K, B, L]: B \in H_{A}\right\}$ is an $\mathscr{H}^{J}$-class contained in $\widehat{H}_{X}^{J}$ for each $K \in \mathscr{M}_{E}$ and $L \in \mathscr{N}_{E}$.

(vi) We have already noted that $\widehat{\mathscr{D}}^{J}=\mathscr{D}^{J}$. We proved (a) while proving (i), above. Parts (b), (c) and (e) were proved in Proposition 6.2. Part (d) follows from (a).

The previous result explains the "inflation" phenomenon discussed at the beginning of this section; see also Figure 8. As an immediate corollary of Theorem 6.4, we may now completely classify the isomorphism classes of finite linear sandwich semigroups.

Theorem 6.5. Let $\mathbb{F}_{1}$ and $\mathbb{F}_{2}$ be two finite fields with $\left|\mathbb{F}_{1}\right|=q_{1}$ and $\left|\mathbb{F}_{2}\right|=q_{2}$, let $m, n, k, l \geq 1$, and let $A \in D_{r}\left(\mathcal{M}_{n m}\right)$ and $B \in D_{s}\left(\mathcal{M}_{l k}\right)$. The following are equivalent:

(i) $\mathcal{M}_{m n}^{A}\left(\mathbb{F}_{1}\right) \cong \mathcal{M}_{k l}^{B}\left(\mathbb{F}_{2}\right)$,

(ii) one of the following holds:

(a) $r=s=0$ and $q_{1}^{m n}=q_{2}^{k l}$, or

(b) $r=s \geq 1,(m, n)=(k, l)$, and $q_{1}=q_{2}$.

Further, if $r \geq 1$, then $\mathcal{M}_{m n}^{A}\left(\mathbb{F}_{1}\right) \cong \mathcal{M}_{k l}^{B}\left(\mathbb{F}_{2}\right)$ if and only if $\operatorname{Reg}\left(\mathcal{M}_{m n}^{A}\left(\mathbb{F}_{1}\right)\right) \cong \operatorname{Reg}\left(\mathcal{M}_{k l}^{B}\left(\mathbb{F}_{2}\right)\right)$.

Proof. Again, if $r \neq s$, then counting the regular $\mathscr{D}^{A}$ - and $\mathscr{D}^{B}$-classes shows that $\mathcal{M}_{m n}^{A}\left(\mathbb{F}_{1}\right) \neq \mathcal{M}_{k l}^{B}\left(\mathbb{F}_{2}\right)$. For the remainder of the proof, we assume $r=s$. Suppose first that $r=s=0$. Then $\mathcal{M}_{m n}^{A}\left(\mathbb{F}_{1}\right)$ and $\mathcal{M}_{k l}^{B}\left(\mathbb{F}_{2}\right)$ are both zero semigroups and so are isomorphic if and only if their sizes, $q_{1}^{m n}$ and $q_{2}^{k l}$, are equal. For the remainder of the proof, we assume $r=s \geq 1$, and write $D_{t}^{A}$ and $D_{t}^{B}$ for the relevant regular $\mathscr{D}^{A_{-}}$ and $\mathscr{D}^{B}$-classes in $\mathcal{M}_{m n}^{A}\left(\mathbb{F}_{1}\right)$ and $\mathcal{M}_{k l}^{B}\left(\mathbb{F}_{2}\right)$ for each $0 \leq t \leq r=s$.

By Theorem 6.4(v), any group $\mathscr{H}^{A}$-class contained in $D_{1}^{A}$ is isomorphic to $\mathcal{G}_{1}\left(\mathbb{F}_{1}\right) \cong \mathbb{F}_{1}^{\times}$, the multiplicative group of $\mathbb{F}_{1}$. Since $\left|\mathbb{F}_{1}^{\times}\right|=q_{1}-1$ and $\left|\mathbb{F}_{2}^{\times}\right|=q_{2}-1$, it follows that if $q_{1} \neq q_{2}$, then $\operatorname{Reg}\left(\mathcal{M}_{m n}^{A}\left(\mathbb{F}_{1}\right)\right) \neq$ $\operatorname{Reg}\left(\mathcal{M}_{k l}^{B}\left(\mathbb{F}_{2}\right)\right)$ and, hence, $\mathcal{M}_{m n}^{A}\left(\mathbb{F}_{1}\right) \neq \mathcal{M}_{k l}^{B}\left(\mathbb{F}_{2}\right)$. Now suppose $q_{1}=q_{2}$ (so $\left.\mathbb{F}_{1} \cong \mathbb{F}_{2}\right)$, and write $q=q_{1}$. By Theorem 6.4(vi), $D_{1}^{A}$ (resp., $D_{1}^{B}$ ) contains $q^{m-r}\left[\begin{array}{l}r \\ 1\end{array}\right]_{q} \mathscr{R}^{A}$-classes (resp., $q^{k-r}\left[\begin{array}{l}r \\ 1\end{array}\right]_{q} \mathscr{R}^{B}$-classes). It follows that if $m \neq k$ (or, dually, if $n \neq l$ ), then $\operatorname{Reg}\left(\mathcal{M}_{m n}^{A}\left(\mathbb{F}_{1}\right)\right) \neq \operatorname{Reg}\left(\mathcal{M}_{k l}^{B}\left(\mathbb{F}_{2}\right)\right)$ and, hence, $\mathcal{M}_{m n}^{A}\left(\mathbb{F}_{1}\right) \neq \mathcal{M}_{k l}^{B}\left(\mathbb{F}_{2}\right)$. Conversely, if (b) holds, then $\mathcal{M}_{m n}^{A}\left(\mathbb{F}_{1}\right) \cong \mathcal{M}_{k l}^{B}\left(\mathbb{F}_{2}\right)$ by Lemma 4.1.ii).

For the final statement, first note that $\mathcal{M}_{m n}^{A}\left(\mathbb{F}_{1}\right) \cong \mathcal{M}_{k l}^{B}\left(\mathbb{F}_{2}\right)$ clearly implies $\operatorname{Reg}\left(\mathcal{M}_{m n}^{A}\left(\mathbb{F}_{1}\right)\right) \cong \operatorname{Reg}\left(\mathcal{M}_{k l}^{B}\left(\mathbb{F}_{2}\right)\right)$. In the previous paragraph, we showed that the negation of $(\mathrm{b}) \operatorname{implies} \operatorname{Reg}\left(\mathcal{M}_{m n}^{A}\left(\mathbb{F}_{1}\right)\right) \neq \operatorname{Reg}\left(\mathcal{M}_{k l}^{B}\left(\mathbb{F}_{2}\right)\right)$. This completes the proof.

Remark 6.6. Of course, if $\operatorname{rank}(A)=\operatorname{rank}(B)=0$, then $\operatorname{Reg}\left(\mathcal{M}_{m n}^{A}\left(\mathbb{F}_{1}\right)\right)=\left\{O_{m n}\right\} \cong \operatorname{Reg}\left(\mathcal{M}_{k l}^{B}\left(\mathbb{F}_{2}\right)\right)=$ $\left\{O_{k l}\right\}$, regardless of $m, n, k, l, q_{1}, q_{2}$. So the final clause of Theorem 6.5 does not hold for $r=0$.

Remark 6.7. The infinite case is not as straight-forward, since $\left|\mathcal{M}_{m n}(\mathbb{F})\right|=|\mathbb{F}|$ for all $m, n \geq 1$, and since it is possible for two non-isomorphic fields $\mathbb{F}_{1}, \mathbb{F}_{2}$ to have isomorphic multiplicative groups $\mathbb{F}_{1}^{\times}, \mathbb{F}_{2}^{\times}$(for example, $\mathbb{Q}$ and $\mathbb{Z}_{3}(x)$ both have multiplicative groups isomorphic to $\mathbb{Z}_{2} \oplus F$, where $F$ is a free abelian group of countably infinite rank). So we have the following isomorphisms: 
(i) $\mathcal{M}_{m n}^{A}\left(\mathbb{F}_{1}\right) \cong \mathcal{M}_{k l}^{B}\left(\mathbb{F}_{2}\right)$ if $m, n, k, l \geq 1,\left|\mathbb{F}_{1}\right|=\left|\mathbb{F}_{2}\right|$, and $\operatorname{rank}(A)=\operatorname{rank}(B)=0$ - indeed, both sandwich semigroups are zero semigroups of size $\left|\mathbb{F}_{1}\right|=\left|\mathbb{F}_{2}\right|$;

(ii) $\mathcal{M}_{m n}^{A}\left(\mathbb{F}_{1}\right) \cong \mathcal{M}_{m n}^{B}\left(\mathbb{F}_{2}\right)$ if $\mathbb{F}_{1}^{\times} \cong \mathbb{F}_{2}^{\times}$and $\operatorname{rank}(A)=\operatorname{rank}(B)=1$ - indeed, when $J=J_{n m 1}=\left[\begin{array}{cc}I_{1} & O \\ O & O\end{array}\right]$, sandwich products $X \star_{J} Y$ involve only field multiplication and no addition:

$$
\left[\begin{array}{cccc}
a_{11} & a_{12} & \cdots & a_{1 n} \\
a_{21} & a_{22} & \cdots & a_{2 n} \\
\vdots & \vdots & \ddots & \vdots \\
a_{m 1} & a_{m 2} & \cdots & a_{m n}
\end{array}\right] \star_{J}\left[\begin{array}{cccc}
b_{11} & b_{12} & \cdots & b_{1 n} \\
b_{21} & b_{22} & \cdots & b_{2 n} \\
\vdots & \vdots & \ddots & \vdots \\
b_{m 1} & b_{m 2} & \cdots & b_{m n}
\end{array}\right]=\left[\begin{array}{cccc}
a_{11} b_{11} & a_{11} b_{12} & \cdots & a_{11} b_{1 n} \\
a_{21} b_{11} & a_{21} b_{12} & \cdots & a_{21} b_{1 n} \\
\vdots & \vdots & \ddots & \vdots \\
a_{m 1} b_{11} & a_{m 1} b_{12} & \cdots & a_{m 1} b_{1 n}
\end{array}\right] .
$$

We leave it as an open problem to completely classify the isomorphism classes of linear sandwich semigroups over infinite fields. But we make two simple observations:

(iii) as in the proof of Theorem 6.5. if $\mathcal{M}_{m n}^{A}\left(\mathbb{F}_{1}\right) \cong \mathcal{M}_{k l}^{B}\left(\mathbb{F}_{2}\right)$, then we must have $\operatorname{rank}(A)=\operatorname{rank}(B)$;

(iv) if $\mathcal{M}_{m n}^{A}\left(\mathbb{F}_{1}\right) \cong \mathcal{M}_{k l}^{B}\left(\mathbb{F}_{2}\right)$ with $\operatorname{rank}(A)=\operatorname{rank}(B)=r \geq 2$, we must have $\mathbb{F}_{1} \cong \mathbb{F}_{2}$ (since the maximal subgroups of $\mathcal{M}_{m n}^{A}\left(\mathbb{F}_{1}\right)$ are isomorphic to $\mathcal{G}_{s}\left(\mathbb{F}_{1}\right)$ for $0 \leq s \leq r$, and since $\mathcal{G}_{s}\left(\mathbb{F}_{1}\right) \cong \mathcal{G}_{s}\left(\mathbb{F}_{2}\right)$ implies $\mathbb{F}_{1} \cong \mathbb{F}_{2}$ for $s \geq 2[17]$ ).

In what follows, the top $\mathscr{D}^{J}$-class of $P=\operatorname{Reg}\left(\mathcal{M}_{m n}^{J}\right)$ plays a special role. We write $D$ for this $\mathscr{D}^{J}$-class, so

$$
D=D_{r}^{J}=\phi^{-1}\left(\mathcal{G}_{r}\right)=\{X \in P: \operatorname{rank}(X)=r\} .
$$

As a special case of Theorem 6.4 (v), $D$ is a $q^{r(m-r)} \times q^{r(n-r)}$ rectangular group with respect to $\mathcal{G}_{r}$. Since $D$ is the pre-image of $\mathcal{G}_{r}$ under the map $\phi: P \rightarrow \mathcal{M}_{r}$, we may think of $D$ as a kind of "inflation" of $\mathcal{G}_{r}$, the group of units of $\mathcal{M}_{r}$. In fact, more can be said along these lines. Recall again that the variant of a semigroup $S$ with respect to an element $a \in S$ is the semigroup $S^{a}$ with underlying set $S$ and operation $\star_{a}$ defined by $x \star_{a} y=x a y$ for all $x, y \in S$. Recall also that an element $a \in S$ of a (necessarily regular) semigroup $S$ is regularity preserving if the variant $S^{a}$ is regular. The set $\operatorname{RP}(S)$ of all regularity preserving elements of $S$ was studied in [38,50]; we will not go into the details here, but it was explained in [50] that $\operatorname{RP}(S)$ is a useful alternative to the group of units in the case that $S$ is not a monoid (as with $P$ when $r=m=n$ does not hold). Because of this, it is significant that $D$ is equal to $\operatorname{RP}(P)$, the set of all regularity preserving elements of $P=\operatorname{Reg}\left(\mathcal{M}_{m n}^{J}\right)$, as we will soon see. We now state a result from [50] concerning regularity preserving elements. Recall that an element $u$ of a semigroup $S$ is a mididentity if $x u y=x y$ for all $x, y \in S$ [98]; of course for such an element, $\star_{u}$ is just the original semigroup operation.

Proposition 6.8 (Khan and Lawson $[50]$ ). Let $S$ be a regular semigroup.

(i) An element $a \in S$ is regularity preserving if and only if a $\mathscr{H}$ e for some regularity preserving idempotent $e \in E(S)$. (In particular, $\operatorname{RP}(S)$ is a union of groups.)

(ii) An idempotent $e \in E(S)$ is regularity preserving if and only if feR $f \mathscr{L}$ ef for all idempotents $f \in E(S)$.

(iii) Any mididentity is regularity preserving.

In order to avoid confusion when discussing idempotents, if $\Omega \subseteq \mathcal{M}_{m n}$, we will write

$$
E_{J}(\Omega)=\{X \in \Omega: X=X \star X\}
$$

for the set of idempotents from $\Omega$ with respect to the $\star$ operation on $\mathcal{M}_{m n}^{J}$. If $\Sigma \subseteq \mathcal{M}_{k}$ for some $k$, we will continue to write $E(\Sigma)=\left\{A \in \Sigma: A=A^{2}\right\}$ for the set of idempotents from $\Sigma$ with respect to the usual matrix multiplication.

Lemma 6.9. (i) $E_{J}\left(\mathcal{M}_{m n}^{J}\right)=E_{J}(P)=\left\{[M, A, N]: A \in E\left(\mathcal{M}_{r}\right), M \in \mathcal{M}_{m-r, r}, N \in \mathcal{M}_{r, n-r}\right\}$.

(ii) $E_{J}(D)=\left\{\left[M, I_{r}, N\right]: M \in \mathcal{M}_{m-r, r}, N \in \mathcal{M}_{r, n-r}\right\}$ is a $q^{r(m-r)} \times q^{r(n-r)}$ rectangular band.

(iii) Each element from $E_{J}(D)$ is a mididentity for both $\mathcal{M}_{m n}^{J}$ and $P$. 
(iv) $D=\operatorname{RP}(P)$ is the set of all regularity-preserving elements of $P$.

Proof. Note that all idempotents are regular. If $X=[M, A, N] \in P$, then $X \star X=\left[M, A^{2}, N\right]$, so $X=X \star X$ if and only if $A=A^{2}$, giving (i). Part (ii) follows from (i), since $I_{r}$ is the only idempotent from the group $\mathcal{G}_{r}=D_{r}\left(\mathcal{M}_{r}\right)$. Using (ii), it is easy to check by direct computation that $X \star Y \star Z=X \star Z$ for all $X, Z \in \mathcal{M}_{m n}$ and $Y \in E_{J}(D)$, giving (iii). Finally, to prove (iv), note that by Proposition 6.8(i), it suffices to show that $E_{J}(\mathrm{RP}(P))=E_{J}(D)$. By (iii) and Proposition 6.8(iii), we have $E_{J}(D) \subseteq E_{J}(\operatorname{RP}(P)$ ). Conversely, suppose $X \in E_{J}(\operatorname{RP}(P))$. Let $Y \in E_{J}(D)$. By Proposition 6.8 (ii), and the fact that $\mathscr{L}^{J} \subseteq \mathscr{D}^{J}$, $X \star Y \mathscr{D}^{J} Y$. It follows that $r=\operatorname{rank}(Y)=\operatorname{rank}(X J Y) \leq \operatorname{rank}(X) \leq r$, giving $\operatorname{rank}(X)=r$, and $X \in E_{J}(D)$. This shows that $E_{J}(\mathrm{RP}(P)) \subseteq E_{J}(D)$, and completes the proof.

We may now calculate the rank of $P=\operatorname{Reg}\left(\mathcal{M}_{m n}^{J}\right)$ in the case of finite $\mathbb{F}$. For the following proof, recall from [46] that the relative $\operatorname{rank} \operatorname{rank}(S: U)$ of a semigroup $S$ with respect to a subset $U \subseteq S$ is defined to be the minimum cardinality of a subset $V \subseteq S$ such that $S=\langle U \cup V\rangle$.

Theorem 6.10. Suppose $|\mathbb{F}|=q<\infty$. If $1 \leq r \leq \min (m, n)$ and we do not have $r=m=n$, then

$$
\operatorname{rank}(P)=\operatorname{rank}\left(\operatorname{Reg}\left(\mathcal{M}_{m n}^{J}\right)\right)=q^{r(L-r)}+1,
$$

where $L=\max (m, n)$.

Proof. Since $D$ is a subsemigroup of $P$ and $P \backslash D$ is an ideal, it quickly follows that $\operatorname{rank}(P)=$ $\operatorname{rank}(D)+\operatorname{rank}(P: D)$. It is well-known 84 that a rectangular group $R=(S \times T) \times G$ satisfies $\operatorname{rank}(R)=\max \{|S|,|T|, \operatorname{rank}(G)\}$. Since $D$ is a $q^{r(m-r)} \times q^{r(n-r)}$ rectangular group with respect to $\mathcal{G}_{r}$, and since $\operatorname{rank}\left(\mathcal{G}_{r}\right) \leq 2$ by Theorem 3.7, it immediately follows that $\operatorname{rank}(D)=q^{r(L-r)}$. Since $\langle D\rangle_{J}=D \neq P$ (as $r \geq 1$ ), we have $\operatorname{rank}(P: D) \geq 1$, so the proof will be complete if we can show that $P=\langle D \cup\{X\}\rangle_{J}$ for some $X \in P$. With this in mind, let $X \in D_{r-1}^{J}$ be arbitrary. Note that $\bar{D}=\{\bar{Y}: Y \in D\}=\mathcal{G}_{r}$, and $\bar{X} \in D_{r-1}\left(\mathcal{M}_{r}\right)$. It follows from Theorem 3.7 that $\mathcal{M}_{r}=\langle\bar{D} \cup\{\bar{X}\}\rangle$. Now let $Y=[M, A, N] \in P$ be arbitrary. Choose $Z_{1}, \ldots, Z_{k} \in D \cup\{X\}$ such that $A=\bar{Z}_{1} \cdots \bar{Z}_{k}$. Then $Y=\left[M, I_{r}, N\right] \star Z_{1} \star \cdots \star Z_{k} \star\left[M, I_{r}, N\right]$, with $\left[M, I_{r}, N\right] \in D$.

Remark 6.11. If $r=0$, then $P=\{O\}$, while if $r=m=n$, then $P=\mathcal{M}_{n}$. So $\operatorname{rank}(P)$ is trivial in the former case, and well-known in the latter (see Theorem 3.7). As in Remark 5.3, we deduce that $\operatorname{rank}\left(\operatorname{Reg}\left(\mathcal{C}_{k}(l)\right)\right)=\operatorname{rank}\left(\operatorname{Reg}\left(\mathcal{R}_{k}(l)\right)\right)=q^{l(k-l)}+1$ for $|\mathbb{F}|=q<\infty$.

\section{The idempotent generated subsemigroup}

In this section, we investigate the idempotent generated subsemigroup $\left\langle E_{J}\left(\mathcal{M}_{m n}^{J}\right)\right\rangle_{J}$ of $\mathcal{M}_{m n}^{J}$; we write $\mathcal{E}_{m n}^{J}$ for this idempotent generated subsemigroup. Our main results include a proof that $\mathcal{E}_{m n}^{J}=(P \backslash D) \cup E_{J}(D)$ and a calculation of $\operatorname{rank}\left(\mathcal{E}_{m n}^{J}\right)$ and $\operatorname{idrank}\left(\mathcal{E}_{m n}^{J}\right)$; in particular, we show that these two values are equal. Since the solution to every problem we consider is trivial when $r=0$, and well-known when $r=m=n$, we will continue to assume that $r \geq 1$ and that $r=m=n$ does not hold. To simplify notation, we will write $E=E_{J}\left(\mathcal{M}_{m n}^{J}\right)=E_{J}(P)$, so $\mathcal{E}_{m n}^{J}=\langle E\rangle_{J}$. We begin by calculating $|E|$ in the case of finite $\mathbb{F}$, for which we need the following formulae for $\left|E\left(D_{s}\left(\mathcal{M}_{r}\right)\right)\right|$. Although the next result might already be known, we are unaware of a reference and include a simple proof for convenience.

Lemma 7.1. Suppose $|\mathbb{F}|=q<\infty$. If $0 \leq s \leq r$, then $\left|E\left(D_{s}\left(\mathcal{M}_{r}\right)\right)\right|=q^{s(r-s)}\left[\begin{array}{c}r \\ s\end{array}\right]_{q}$. Consequently,

$$
\left|E\left(\mathcal{M}_{r}\right)\right|=\sum_{s=0}^{r} q^{s(r-s)}\left[\begin{array}{l}
r \\
s
\end{array}\right]_{q} .
$$


Proof. To specify an idempotent endomorphism $\alpha \in \operatorname{End}\left(V_{r}\right)$ of rank $s$, we first choose $W=\operatorname{im}(\alpha)$, which is a subspace of dimension $s$ and may be chosen in $\left[\begin{array}{c}r \\ s\end{array}\right]_{q}$ ways, and we note that $\alpha$ must map $W$ identically. If $\left\{v_{1}, \ldots, v_{r}\right\}$ is an arbitrary basis for $V_{r}$, such that $\left\{v_{1}, \ldots, v_{s}\right\}$ is a basis of $W$, then $\alpha$ may map each of $v_{s+1}, \ldots, v_{r}$ arbitrarily into $W$, and there are $\left(q^{s}\right)^{r-s}$ ways to choose these images.

Proposition 7.2. Suppose $|\mathbb{F}|=q<\infty$. If $0 \leq s \leq r$, then $\left|E_{J}\left(D_{s}^{J}\right)\right|=q^{s(m+n-r-s)}\left[\begin{array}{l}r \\ s\end{array}\right]_{q}$. Consequently,

$$
\left|E_{J}\left(\mathcal{M}_{m n}^{J}\right)\right|=\sum_{s=0}^{r} q^{s(m+n-r-s)}\left[\begin{array}{l}
r \\
s
\end{array}\right]_{q} .
$$

Proof. Parts (iv) and (v) of Theorem 6.4 say that an $\mathscr{H}^{J}$-class $H_{X}^{J} \subseteq D_{s}^{J}$ is a group (so contains an idempotent) if and only if $H_{\bar{X}}$ is a group $\mathscr{H}$-class of $\mathcal{M}_{r}$, and that there are $q^{s(m-r)} \times q^{s(n-r)}$ idempotents of $D_{s}^{J}$ corresponding to each rank $s$ idempotent of $\mathcal{M}_{r}$, of which there are $q^{s(r-s)}\left[{ }_{s}^{r}\right]_{q}$ by Lemma 7.1. The result quickly follows.

We now describe the idempotent generated subsemigroup of $\mathcal{M}_{m n}^{J}$.

Theorem 7.3. We have $\mathcal{E}_{m n}^{J}=\left\langle E_{J}\left(\mathcal{M}_{m n}^{J}\right)\right\rangle_{J}=(P \backslash D) \cup E_{J}(D)$.

Proof. Suppose $X_{1}, \ldots, X_{k} \in E=E_{J}\left(\mathcal{M}_{m n}^{J}\right)$, and write $X_{i}=\left[M_{i}, A_{i}, N_{i}\right]$ for each $i$. So $A_{i} \in E\left(\mathcal{M}_{r}\right)$ for each $i$. Then $X_{1} \star \cdots \star X_{k}=\left[M_{1}, A_{1} \cdots A_{k}, N_{k}\right]$. If any of $A_{1}, \ldots, A_{k}$ belongs to $\mathcal{M}_{r} \backslash \mathcal{G}_{r}$, then so too does $A_{1} \cdots A_{k}$, so that $X_{1} \star \cdots \star X_{k} \in P \backslash D$. If all of $A_{1}, \ldots, A_{k}$ belong to $\mathcal{G}_{r}$, then $A_{1}=\cdots=A_{k}=I_{r}$, so $X_{1} \star \cdots \star X_{k}=\left[M_{1}, I_{r}, N_{k}\right] \in E_{J}(D)$. This shows that $\mathcal{E}_{m n}^{J} \subseteq(P \backslash D) \cup E_{J}(D)$. Conversely, it suffices to show that $P \backslash D \subseteq \mathcal{E}_{m n}^{J}$, so suppose $X \in P \backslash D$, and write $X=[M, A, N]$. Since $X \notin D$, we must have $\operatorname{rank}(A)=\operatorname{rank}(X)<r$. But then $A \in \mathcal{M}_{r} \backslash \mathcal{G}_{r}$, so that $A=B_{1} \cdots B_{l}$ for some $B_{1}, \ldots, B_{l} \in E\left(\mathcal{M}_{r}\right)$ by Theorem 3.5. It follows that $X=\left[M, B_{1}, N\right] \star \cdots \star\left[M, B_{l}, N\right]$, with all $\left[M, B_{i}, N\right] \in E$.

Remark 7.4. Recall (see Theorem 3.5 that $\mathcal{E}_{n}=\left\langle E\left(\mathcal{M}_{n}\right)\right\rangle=\left(\mathcal{M}_{n} \backslash \mathcal{G}_{n}\right) \cup\left\{I_{n}\right\}$. Theorem 7.3 is a pleasing analogue of that result, since $\left\{I_{n}\right\}=E\left(\mathcal{G}_{n}\right)$, where $\mathcal{G}_{n}$ is the top $\mathscr{D}$-class of $\mathcal{M}_{n}$. Also, $\mathcal{G}_{n}=G\left(\mathcal{M}_{n}\right)=$ $\operatorname{RP}\left(\mathcal{M}_{n}\right)$ and, while $P$ has no group of units as it is not a monoid, it is still the case that $D=\operatorname{RP}(P)$.

Now that we have described the elements of the semigroup $\mathcal{E}_{m n}^{J}$, the next natural task is to calculate its rank and idempotent rank.

Theorem 7.5. Suppose $|\mathbb{F}|=q<\infty$. Then

$$
\operatorname{rank}\left(\mathcal{E}_{m n}^{J}\right)=\operatorname{idrank}\left(\mathcal{E}_{m n}^{J}\right)=q^{r(L-r)}+\left(q^{r}-1\right) /(q-1),
$$

where $L=\max (m, n)$.

Proof. As in the proof of Theorem 6.10, we have $\operatorname{rank}\left(\mathcal{E}_{m n}^{J}\right)=\operatorname{rank}\left(E_{J}(D)\right)+\operatorname{rank}\left(\mathcal{E}_{m n}^{J}: E_{J}(D)\right)$. Since $E_{J}(D)$ is a $q^{r(m-r)} \times q^{r(n-r)}$ rectangular band (see Lemma 6.9 (ii)), we again deduce from 84 that $\operatorname{rank}\left(E_{J}(D)\right)=\operatorname{idrank}\left(E_{J}(D)\right)=q^{r(L-r)}$. So it remains to show that:

(i) there exists a set $\Omega \subseteq E$ of size $\left(q^{r}-1\right) /(q-1)$ such that $\mathcal{E}_{m n}^{J}=\left\langle E_{J}(D) \cup \Omega\right\rangle_{J}$, and

(ii) if $\Sigma \subseteq P$ satisfies $\mathcal{E}_{m n}^{J}=\left\langle E_{J}(D) \cup \Sigma\right\rangle_{J}$, then $|\Sigma| \geq\left(q^{r}-1\right) /(q-1)$.

By Theorem 3.5, we may choose some set $\Gamma \subseteq E\left(\mathcal{M}_{r}\right)$ with $\langle\Gamma\rangle=\mathcal{M}_{r} \backslash \mathcal{G}_{r}$ and $|\Gamma|=\left(q^{r}-1\right) /(q-1)$. For each $A \in \Gamma$, choose any $M_{A} \in \mathcal{M}_{m-r, r}$ and $N_{A} \in \mathcal{M}_{r, n-r}$, and put $\Omega=\left\{\left[M_{A}, A, N_{A}\right]: A \in \Gamma\right\}$. Since $\mathcal{E}_{m n}^{J}=(P \backslash D) \cup E_{J}(D)$, the proof of (i) will be complete if we can show that $P \backslash D \subseteq\left\langle E_{J}(D) \cup \Omega\right\rangle_{J}$. So let $X=[K, B, L] \in P \backslash D$, and write $B=A_{1} \cdots A_{k}$ where $A_{1}, \ldots, A_{k} \in \Gamma$. Then

$$
X=\left[K, I_{r}, L\right] \star\left[M_{A_{1}}, A_{1}, N_{A_{1}}\right] \star \cdots \star\left[M_{A_{k}}, A_{k}, N_{A_{k}}\right] \star\left[K, I_{r}, L\right] \in\left\langle E_{J}(D) \cup \Omega\right\rangle_{J},
$$


as required. Next, suppose $\mathcal{E}_{m n}^{J}=\left\langle E_{J}(D) \cup \Sigma\right\rangle_{J}$, where $\Sigma \subseteq \mathcal{E}_{m n}^{J} \backslash E_{J}(D)=P \backslash D$. We will show that $\bar{\Sigma}$ generates $\mathcal{M}_{r} \backslash \mathcal{G}_{r}$. Indeed, let $A \in \mathcal{M}_{r} \backslash \mathcal{G}_{r}$ be arbitrary, and choose any $X \in P$ such that $\bar{X}=A$. Since $\operatorname{rank}(X)=\operatorname{rank}(A)<r$, it follows that $X \in P \backslash D \subseteq \mathcal{E}_{m n}^{J}$. Consider an expression $X=Y_{1} \star \cdots \star Y_{k}$, where $Y_{1}, \ldots, Y_{k} \in E_{J}(D) \cup \Sigma$. Now, $A=\bar{X}=\bar{Y}_{1} \ldots \bar{Y}_{k}$. If any of the $Y_{i}$ belongs to $E_{J}(D)$, then $\bar{Y}_{i}=I_{r}$, so the factor $\bar{Y}_{i}$ is not needed in the product $A=\bar{Y}_{1} \cdots \bar{Y}_{k}$. After cancelling all such factors, we see that $A$ is a product of elements from $\bar{\Sigma}$. Since $A \in \mathcal{M}_{r} \backslash \mathcal{G}_{r}$ was arbitrary, we conclude that $\mathcal{M}_{r} \backslash \mathcal{G}_{r}=\langle\bar{\Sigma}\rangle$. In particular, $|\Sigma| \geq|\bar{\Sigma}| \geq \operatorname{rank}\left(\mathcal{M}_{r} \backslash \mathcal{G}_{r}\right)=\left(q^{r}-1\right) /(q-1)$, giving (ii).

Remark 7.6. As in Remarks 5.3 and 6.11, we deduce from the results of this section that for $|\mathbb{F}|=q<\infty$,

- $\mathcal{C}_{k}(l)$ (and $\left.\mathcal{R}_{k}(l)\right)$ has $\sum_{s=0}^{l} q^{s(k-s)}\left[\begin{array}{l}l \\ s\end{array}\right]_{q}$ idempotents,

- the semigroup generated by $E\left(\mathcal{C}_{k}(l)\right)$ (and the semigroup generated by $E\left(\mathcal{R}_{k}(l)\right)$ ) has rank and idempotent rank equal to $q^{l(k-l)}+\left(q^{l}-1\right) /(q-1)$.

\section{Ideals}

In this final section, we consider the ideals of $P=\operatorname{Reg}\left(\mathcal{M}_{m n}^{J}\right)$. In particular, we show that each of the proper ideals is idempotent generated, and we calculate the rank and idempotent rank, showing that these are equal. Although the next result is trivial if $r=0$ and well-known if $r=m=n$ (see Theorem 3.6), the statement is valid for those parameters.

Theorem 8.1. The ideals of $P=\operatorname{Reg}\left(\mathcal{M}_{m n}^{J}\right)$ are precisely the sets

$$
I_{s}^{J}=D_{0}^{J} \cup \cdots \cup D_{s}^{J}=\{X \in P: \operatorname{rank}(X) \leq s\} \quad \text { for } 0 \leq s \leq r,
$$

and they form a chain: $I_{0}^{J} \subseteq \cdots \subseteq I_{r}^{J}$. If $0 \leq s<r$, then $I_{s}^{J}=\left\langle E_{J}\left(D_{s}^{J}\right)\right\rangle_{J}$ is generated by the idempotents in its top $\mathscr{D}^{J}$-class, and if $|\mathbb{F}|=q<\infty$, then

$$
\operatorname{rank}\left(I_{s}^{J}\right)=\operatorname{idrank}\left(I_{s}^{J}\right)=q^{s(L-r)}\left[\begin{array}{l}
r \\
s
\end{array}\right]_{q}, \quad \text { where } L=\max (m, n) .
$$

Proof. For convenience, we will assume that $m \leq n$ throughout the proof, so that $L=n$. (The other case will follow by duality.)

More generally, it may easily be checked that if the $\mathscr{J}$-classes of a semigroup $S$ form a chain, $J_{0}<\cdots<J_{k}$, then the ideals of $S$ are precisely the sets $I_{h}=J_{0} \cup \cdots \cup J_{h}$ for $0 \leq h \leq k$ (and these obviously form a chain). Now suppose $0 \leq s<r$, let $\Gamma \subseteq E\left(D_{s}\left(\mathcal{M}_{r}\right)\right)$ be any idempotent generating set of $I_{s}\left(\mathcal{M}_{r}\right)$ (see Theorem 3.6), and put $\Omega_{\Gamma}=\left\{[M, A, N]: M \in \mathcal{M}_{m-r, r}, A \in \Gamma, N \in \mathcal{M}_{r, n-r}\right\}$. If $X=[M, A, N] \in I_{s}^{J}$ is arbitrary, then $A=B_{1} \cdots B_{k}$ for some $B_{1}, \ldots, B_{k} \in \Gamma$, and it follows that $X=\left[M, B_{1}, N\right] \star \cdots \star\left[M, B_{k}, N\right] \in\left\langle\Omega_{\Gamma}\right\rangle_{J}$. Since $\Omega_{\Gamma} \subseteq E_{J}\left(D_{s}^{J}\right)$, it follows that $I_{s}^{J}=\left\langle E_{J}\left(D_{s}^{J}\right)\right\rangle_{J}$.

We now prove the statement about rank and idempotent rank. Suppose $\Omega$ is an arbitrary generating set for $I_{s}^{J}$ where $0 \leq s<r$. Let $X \in D_{s}^{J}$ and consider an expression $X=Y_{1} \star \cdots \star Y_{k}$ with $Y_{1}, \ldots, Y_{k} \in \Omega$. Since $X=X \star Z \star X$ for some $Z \in D_{s}^{J}$, we may assume that $k \geq 2$. Since $I_{s-1}^{J}$ is an ideal of $I_{s}^{J}$ (we interpret $I_{s-1}^{J}=\emptyset$ if $\left.s=0\right)$, each of $Y_{1}, \ldots, Y_{k}$ must belong to $D_{s}^{J}=D_{X}^{J}$. In particular, $Y_{k} \mathscr{D}^{J} X=\left(Y_{1} \star \cdots \star Y_{k-1}\right) \star Y_{k}$. By stability, it then follows that $Y_{k} \mathscr{L}^{J}\left(Y_{1} \star \cdots \star Y_{k-1}\right) \star Y_{k}=X$. Since $X \in D_{s}^{J}$ was arbitrary, it follows that $\Omega$ contains at least one element from each $\mathscr{L}^{J}$-class contained in $D_{s}^{J}$, and there are $q^{s(n-r)}\left[\begin{array}{l}r \\ s\end{array}\right]_{q}$ such $\mathscr{L}^{J}$-classes, by Theorem 6.4(vi). It follows that $\operatorname{rank}\left(I_{s}^{J}\right) \geq q^{s(n-r)}\left[\begin{array}{l}r \\ s\end{array}\right]_{q}=q^{s(L-r)}\left[\begin{array}{l}r \\ s\end{array}\right]_{q}$.

Since $\operatorname{idrank}(S) \geq \operatorname{rank}(S)$ for any idempotent generated semigroup $S$, the proof will be complete if we can find an idempotent generating set of $I_{s}^{J}$ of the specified size. First, let $\Gamma \subseteq E\left(D_{s}\left(\mathcal{M}_{r}\right)\right)$ be such that $\langle\Gamma\rangle=I_{s}\left(\mathcal{M}_{r}\right)$ and $|\Gamma|=\left[\begin{array}{l}r \\ s\end{array}\right]_{q}$. Fix some $A \in \Gamma$, and let $\sim_{A}$ and $\approx_{A}$ be the equivalence relations on $\mathcal{M}_{m-r, r}$ and $\mathcal{M}_{r, n-r}$ defined before Theorem 6.4, and let $\mathscr{M}_{A}$ and $\mathscr{N}_{A}$ be cross-sections of the equivalence classes of $\sim_{A}$ and $\approx_{A}$. Let $\mathscr{M}_{A}=\left\{M_{1}, \ldots, M_{q^{s(m-r)}}\right\}$ and $\mathscr{N}_{A}=\left\{N_{1}, \ldots, N_{q^{s(n-r)}}\right\}$. (We know $\mathscr{M}_{A}$ and 
$\mathscr{N}_{A}$ have the specified sizes by Theorem 6.4.) Put $Q=q^{s(n-r)}=q^{s(L-r)}$. (Recall that we are assuming $m \leq n$.) Extend $\mathscr{M}_{A}$ arbitrarily to $\mathscr{M}_{A}^{\prime}=\left\{M_{1}, \ldots, M_{Q}\right\}$. Now put $\Omega_{A}=\left\{\left[M_{i}, A, N_{i}\right]: 1 \leq i \leq Q\right\}$. If $M \in \mathcal{M}_{m-r, r}$ and $N \in \mathcal{M}_{r, n-r}$ are arbitrary, then $M \sim M_{i}$ and $N \sim N_{j}$ for some $i, j$, and we have $[M, A, N]=\left[M_{i}, A, N_{j}\right]=\left[M_{i}, A, N_{i}\right] \star\left[M_{j}, A, N_{j}\right] \in\left\langle\Omega_{A}\right\rangle_{J}$. Now put $\Omega=\bigcup_{A \in \Gamma} \Omega_{A}$. By the previous discussion, we see that $\langle\Omega\rangle_{J}$ contains $\Omega_{\Gamma}$, which is a generating set for $I_{s}^{J}$ (by the first paragraph of this proof), so $I_{s}^{J}=\langle\Omega\rangle_{J}$. Since $|\Omega|=Q|\Gamma|=q^{s(L-r)}\left[\begin{array}{l}r \\ s\end{array}\right]_{q}$, the proof is complete.

Remark 8.2. Again, we may deduce a corresponding statement for the ideals of the matrix semigroups $\operatorname{Reg}\left(\mathcal{C}_{k}(l)\right)$ and $\operatorname{Reg}\left(\mathcal{R}_{k}(l)\right)$; the reader may supply the details if they wish.

\section{Acknowledgements}

The first named author gratefully acknowledges the support of Grant No. 174019 of the Ministry of Education, Science, and Technological Development of the Republic of Serbia, and Grant No. 1136/2014 of the Secretariat of Science and Technological Development of the Autonomous Province of Vojvodina. The authors wish to thank Dr Attila Egri-Nagy for constructing the GAP [67] code that enabled us to produce the eggbox diagrams from Figures 4, 5,7 and 8 .

\section{References}

[1] Jorge Almeida, Stuart Margolis, Benjamin Steinberg, and Mikhail Volkov. Representation theory of finite semigroups, semigroup radicals and formal language theory. Trans. Amer. Math. Soc., 361(3):1429-1461, 2009.

[2] J. Araújo and J. D. Mitchell. An elementary proof that every singular matrix is a product of idempotent matrices. Amer. Math. Monthly, 112(7):641-645, 2005.

[3] Richard Brauer. On algebras which are connected with the semisimple continuous groups. Ann. of Math. (2), 38(4):857-872, 1937.

[4] Thomas Breuer, Robert M. Guralnick, and William M. Kantor. Probabilistic generation of finite simple groups. II. J. Algebra, 320(2):443-494, 2008.

[5] W. P. Brown. Generalized matrix algebras. Canad. J. Math., 7:188-190, 1955.

[6] William P. Brown. The semisimplicity of $\omega_{f}^{n}$. Ann. of Math. (2), 63:324-335, 1956.

[7] Phatsarapa Chanmuang and Ronnason Chinram. Some remarks on regularity of generalized transformation semigroups. Int. J. Algebra, 2(9-12):581-584, 2008.

[8] Karen Chase. Sandwich semigroups of binary relations. Discrete Math., 28(3):231-236, 1979.

[9] R. Chinram. Green's relations and regularity of generalized semigroups of linear transformations. Lobachevskii J. Math., 30(4):253-256, 2009.

[10] Ronnason Chinram. Regularity and Green's relations of generalized one-to-one partial transformation semigroups. Far East J. Math. Sci., 30(3):513-521, 2008.

[11] Ronnason Chinram. Regularity and Green's relations of generalized partial transformation semigroups. Asian-Eur. J. Math., 1(3):295-302, 2008.

[12] A. H. Clifford. Matrix representations of completely simple semigroups. Amer. J. Math., 64:327-342, 1942.

[13] A. H. Clifford. Basic representations of completely simple semigroups. Amer. J. Math., 82:430-434, 1960.

[14] A. H. Clifford and G. B. Preston. The algebraic theory of semigroups. Vol. I. Mathematical Surveys, No. 7. American Mathematical Society, Providence, R.I., 1961.

[15] R. J. H. Dawlings. Products of idempotents in the semigroup of singular endomorphisms of a finite-dimensional vector space. Proc. Roy. Soc. Edinburgh Sect. A, 91(1-2):123-133, 1981/82.

[16] R. J. H. Dawlings. Sets of idempotents that generate the semigroup of singular endomorphisms of a finite-dimensional vector space. Proc. Edinburgh Math. Soc. (2), 25(2):133-139, 1982.

[17] Jean A. Dieudonné. La géométrie des groupes classiques (in French). Springer-Verlag, Berlin-New York, 1971. Troisième édition, Ergebnisse der Mathematik und ihrer Grenzgebiete, Band 5.

[18] D. Ž. Djoković. Note on a theorem on singular matrices. Canad. Math. Bull., 11:283-284, 1968.

[19] Igor Dolinka and James East. Variants of finite full transformation semigroups. Internat. J. Algebra Comput., 25(8):11871222, 2015.

[20] Igor Dolinka and Robert D. Gray. Maximal subgroups of free idempotent generated semigroups over the full linear monoid. Trans. Amer. Math. Soc., 366(1):419-455, 2014.

[21] Jie Du and Zongzhu Lin. Stratifying algebras with near-matrix algebras. J. Pure Appl. Algebra, 188(1-3):59-72, 2004.

[22] D. Easdown and T. G. Lavers. The inverse braid monoid. Adv. Math., 186(2):438-455, 2004.

[23] J. East, J. D. Mitchell, and Y. Péresse. Maximal subsemigroups of the semigroup of all mappings on an infinite set. Trans. Amer. Math. Soc., 367(3):1911-1944, 2015.

[24] Charles Ehresmann. Catégories et structures (in French). Dunod, Paris, 1965.

[25] J. A. Erdos. On products of idempotent matrices. Glasgow Math. J., 8:118-122, 1967. 
[26] D. G. FitzGerald and Jonathan Leech. Dual symmetric inverse monoids and representation theory. J. Austral. Math. Soc. Ser. A, 64(3):345-367, 1998.

[27] John Fountain and Andrew Lewin. Products of idempotent endomorphisms of an independence algebra of finite rank. Proc. Edinburgh Math. Soc. (2), 35(3):493-500, 1992.

[28] Olexandr Ganyushkin and Volodymyr Mazorchuk. Classical finite transformation semigroups, an introduction, volume 9 of Algebra and Applications. Springer-Verlag London, Ltd., London, 2009.

[29] Olexandr Ganyushkin, Volodymyr Mazorchuk, and Benjamin Steinberg. On the irreducible representations of a finite semigroup. Proc. Amer. Math. Soc., 137(11):3585-3592, 2009.

[30] Fabio Gavarini. On the radical of Brauer algebras. Math. Z., 260(3):673-697, 2008.

[31] Nick Gill. On a conjecture of Degos. Preprint, 2015, arXiv:1502.03341.

[32] Gracinda Gomes and John M. Howie. On the ranks of certain finite semigroups of transformations. Math. Proc. Cambridge Philos. Soc., 101(3):395-403, 1987.

[33] R. Gray. Hall's condition and idempotent rank of ideals of endomorphism monoids. Proc. Edinb. Math. Soc. (2), 51(1):57$72,2008$.

[34] R. Gray and N. Ruškuc. Maximal subgroups of free idempotent-generated semigroups over the full transformation monoid. Proc. Lond. Math. Soc. (3), 104(5):997-1018, 2012.

[35] Nicolas Guay and Stewart Wilcox. Almost cellular algebras. J. Pure Appl. Algebra, 219(9):4105-4116, 2015.

[36] Robert M. Guralnick and William M. Kantor. Probabilistic generation of finite simple groups. J. Algebra, 234(2):743-792, 2000. Special issue in honor of Helmut Wielandt.

[37] T. E. Hall. The radical of the algebra of any finite semigroup over any field. J. Austral. Math. Soc., 11:350-352, 1970.

[38] J. B. Hickey. Semigroups under a sandwich operation. Proc. Edinburgh Math. Soc. (2), 26(3):371-382, 1983.

[39] J. B. Hickey. On variants of a semigroup. Bull. Austral. Math. Soc., 34(3):447-459, 1986.

[40] Peter M. Higgins. Techniques of semigroup theory. Oxford Science Publications. The Clarendon Press, Oxford University Press, New York, 1992.

[41] Christopher Hollings. The Ehresmann-Schein-Nambooripad theorem and its successors. Eur. J. Pure Appl. Math., 5(4):414450, 2012.

[42] J. M. Howie. The subsemigroup generated by the idempotents of a full transformation semigroup. J. London Math. Soc., 41:707-716, 1966.

[43] J. M. Howie. Idempotent generators in finite full transformation semigroups. Proc. Roy. Soc. Edinburgh Sect. A, 81(34):317-323, 1978.

[44] John M. Howie. Fundamentals of semigroup theory, volume 12 of London Mathematical Society Monographs. New Series. The Clarendon Press, Oxford University Press, New York, 1995. Oxford Science Publications.

[45] John M. Howie and Robert B. McFadden. Idempotent rank in finite full transformation semigroups. Proc. Roy. Soc. Edinburgh Sect. A, 114(3-4):161-167, 1990.

[46] John M. Howie, N. Ruškuc, and P. M. Higgins. On relative ranks of full transformation semigroups. Comm. Algebra, 26(3):733-748, 1998.

[47] Zur Izhakian, John Rhodes, and Benjamin Steinberg. Representation theory of finite semigroups over semirings. J. Algebra, 336:139-157, 2011.

[48] R. Jongchotinon, S. Chaopraknoi, and Y. Kemprasit. Isomorphism theorems for variants of semigroups of linear transformations. Int. J. Algebra, 4(25-28):1407-1412, 2010.

[49] Yupaporn Kemprasit. Regularity and unit-regularity of generalized semigroups of linear transformations. Southeast Asian Bull. Math., 25(4):617-622, 2002.

[50] T. A. Khan and M. V. Lawson. Variants of regular semigroups. Semigroup Forum, 62(3):358-374, 2001.

[51] Steffen König and Changchang Xi. On the structure of cellular algebras. In Algebras and modules, II (Geiranger, 1996), volume 24 of CMS Conf. Proc., pages 365-386. Amer. Math. Soc., Providence, RI, 1998.

[52] Steffen König and Changchang Xi. A characteristic free approach to Brauer algebras. Trans. Amer. Math. Soc., 353(4):14891505, 2001.

[53] Thomas J. Laffey. Products of idempotent matrices. Linear and Multilinear Algebra, 14(4):309-314, 1983.

[54] Gérard Lallement and Mario Petrich. Irreducible matrix representations of finite semigroups. Trans. Amer. Math. Soc., 139:393-412, 1969.

[55] Yanbo Li and Feng Wei. Semi-centralizing maps of generalized matrix algebras. Linear Algebra Appl., 436(5):1122-1153, 2012.

[56] Markus Linckelmann and Michał Stolorz. On simple modules over twisted finite category algebras. Proc. Amer. Math. Soc., 140(11):3725-3737, 2012.

[57] E. S. Lyapin. Semigroups (in Russian). Gosudarstv. Izdat. Fiz.-Mat. Lit., Moscow, 1960.

[58] K. D. Magill, Jr. and S. Subbiah. Green's relations for regular elements of sandwich semigroups. I. General results. Proc. London Math. Soc. (3), 31(2):194-210, 1975.

[59] K. D. Magill, Jr. and S. Subbiah. Green's relations for regular elements of sandwich semigroups. II. Semigroups of continuous functions. J. Austral. Math. Soc. Ser. A, 25(1):45-65, 1978.

[60] Kenneth D. Magill, Jr. Semigroup structures for families of functions. I. Some homomorphism theorems. J. Austral. Math. Soc., 7:81-94, 1967.

[61] Paul Martin. Temperley-Lieb algebras for nonplanar statistical mechanics - the partition algebra construction. J. Knot Theory Ramifications, 3(1):51-82, 1994.

[62] D. B. McAlister. The category of representations of a completely 0-simple semigroup. J. Austral. Math. Soc., 12:193-210, 1971.

[63] D. B. McAlister. Rings related to completely 0-simple semigroups. J. Austral. Math. Soc., 12:257-274, 1971. 
[64] Donald B. McAlister. Representations of semigroups by linear transformations. I, II. Semigroup Forum 2 (1971), no. 3, 189-263; ibid., 2(4):283-320, 1971.

[65] Suzana Mendes-Gonçalves and R. P. Sullivan. Regular elements and Green's relations in generalized transformation semigroups. Asian-Eur. J. Math., 6(1):1350006, 11, 2013.

[66] Suzana Mendes-Gonçalves and R. P. Sullivan. Regular elements and Green's relations in generalised linear transformation semigroups. Southeast Asian Bull. Math., 38(1):73-82, 2014.

[67] J. D. Mitchell. The Semigroups package for GAP, Version 2.1. http://tinyurl.com/semigroups, 2014.

[68] W. D. Munn. On semigroup algebras. Proc. Cambridge Philos. Soc., 51:1-15, 1955.

[69] W. D. Munn. Matrix representations of semigroups. Proc. Cambrdige Philos. Soc., 53:5-12, 1957.

[70] W. D. Munn. Irreducible matrix representations of semigroups. Quart. J. Math. Oxford Ser. (2), 11:295-309, 1960.

[71] Attila Nagy. Special classes of semigroups, volume 1 of Advances in Mathematics (Dordrecht). Kluwer Academic Publishers, Dordrecht, 2001.

[72] S. Nenthein and Y. Kemprasit. Regular elements of some semigroups of linear transformations and matrices. Int. Math. Forum, 2(1-4):155-166, 2007.

[73] Jan Okniński. Semigroup algebras, volume 138 of Monographs and Textbooks in Pure and Applied Mathematics. Marcel Dekker, Inc., New York, 1991.

[74] Jan Okniński. Semigroups of matrices, volume 6 of Series in Algebra. World Scientific Publishing Co., Inc., River Edge, NJ, 1998.

[75] Jan Okniński and Mohan S. Putcha. Complex representations of matrix semigroups. Trans. Amer. Math. Soc., 323(2):563581, 1991.

[76] I. S. Ponizovskiur. On matrix representations of associative systems. Mat. Sb. N.S., 38(80):241-260, 1956.

[77] Mohan S. Putcha. Linear algebraic monoids, volume 133 of London Mathematical Society Lecture Note Series. Cambridge University Press, Cambridge, 1988.

[78] Mohan S. Putcha. Complex representations of finite monoids. Proc. London Math. Soc. (3), 73(3):623-641, 1996.

[79] Mohan S. Putcha. Complex representations of finite monoids. II. Highest weight categories and quivers. J. Algebra, 205(1):53-76, 1998.

[80] Mohan S. Putcha. Products of idempotents in algebraic monoids. J. Aust. Math. Soc., 80(2):193-203, 2006.

[81] D. Rees. On semi-groups. Proc. Cambridge Philos. Soc., 36:387-400, 1940.

[82] Lex E. Renner. Linear algebraic monoids, volume 134 of Encyclopaedia of Mathematical Sciences. Springer-Verlag, Berlin, 2005. Invariant Theory and Algebraic Transformation Groups, V.

[83] John Rhodes and Benjamin Steinberg. The q-theory of finite semigroups. Springer Monographs in Mathematics. Springer, New York, 2009.

[84] N. Ruškuc. On the rank of completely 0-simple semigroups. Math. Proc. Cambridge Philos. Soc., 116(2):325-338, 1994.

[85] Benjamin Steinberg. Möbius functions and semigroup representation theory. J. Combin. Theory Ser. A, 113(5):866-881, 2006.

[86] Benjamin Steinberg. Möbius functions and semigroup representation theory. II. Character formulas and multiplicities. Adv. Math., 217(4):1521-1557, 2008.

[87] Benjamin Steinberg. The representation theory of finite monoids. Universitext. Springer, In press.

[88] R. P. Sullivan. Generalised partial transformation semigroups. J. Austral. Math. Soc., 19(part 4):470-473, 1975.

[89] R. P. Sullivan. Semigroups of linear transformations with restricted range. Bull. Aust. Math. Soc., 77(3):441-453, 2008.

[90] R. P. Sullivan. Generalised transformation semigroups. Preprint, 2013.

[91] Melvin C. Thornton. Regular elements in sandwich semigroups of binary relations. Discrete Math., 41(3):303-307, 1982.

[92] R. M. Thrall. A class of algebras without unity element. Canad. J. Math., 7:382-390, 1955.

[93] Amorn Wasanawichit and Yupaporn Kemprasit. Dense subsemigroups of generalised transformation semigroups. J. Aust. Math. Soc., 73(3):433-445, 2002.

[94] William C. Waterhouse. Two generators for the general linear groups over finite fields. Linear and Multilinear Algebra, 24(4):227-230, 1989.

[95] Hermann Weyl. The Classical Groups. Their Invariants and Representations. Princeton University Press, Princeton, N.J., 1939.

[96] Zhankui Xiao and Feng Wei. Commuting mappings of generalized matrix algebras. Linear Algebra Appl., 433(11-12):21782197, 2010.

[97] Zhankui Xiao and Feng Wei. Commuting traces and Lie isomorphisms on generalized matrix algebras. Oper. Matrices, $8(3): 821-847,2014$.

[98] Miyuki Yamada. A note on middle unitary semigroups. Kōdai Math. Sem. Rep., 7:49-52, 1955. 